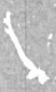
deage Lee

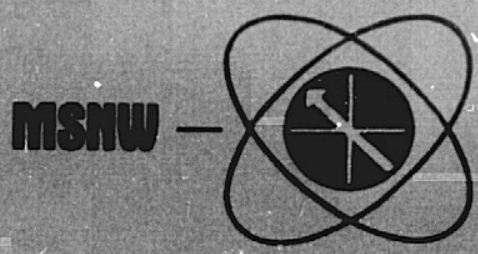

\title{
STUDY, OPTIMIZATION, AND DESIGN of a laser heat eNGINE
}

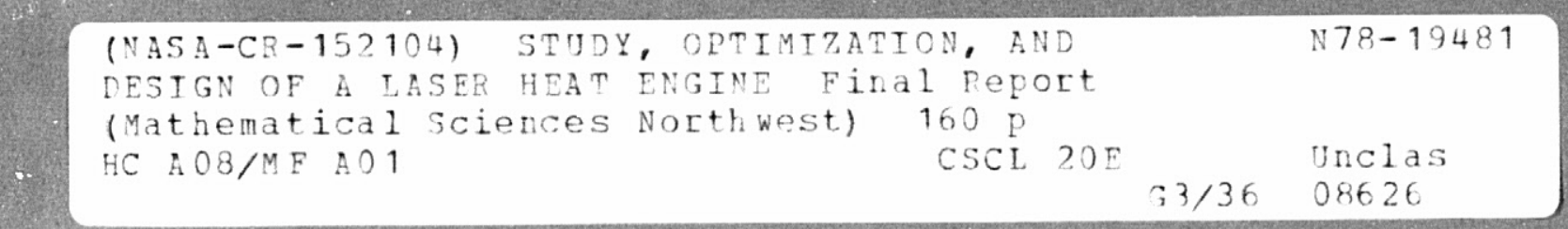

\section{Prepared for}

\section{NASAAMES RESEARCH CENTER}

12 January 1978

u
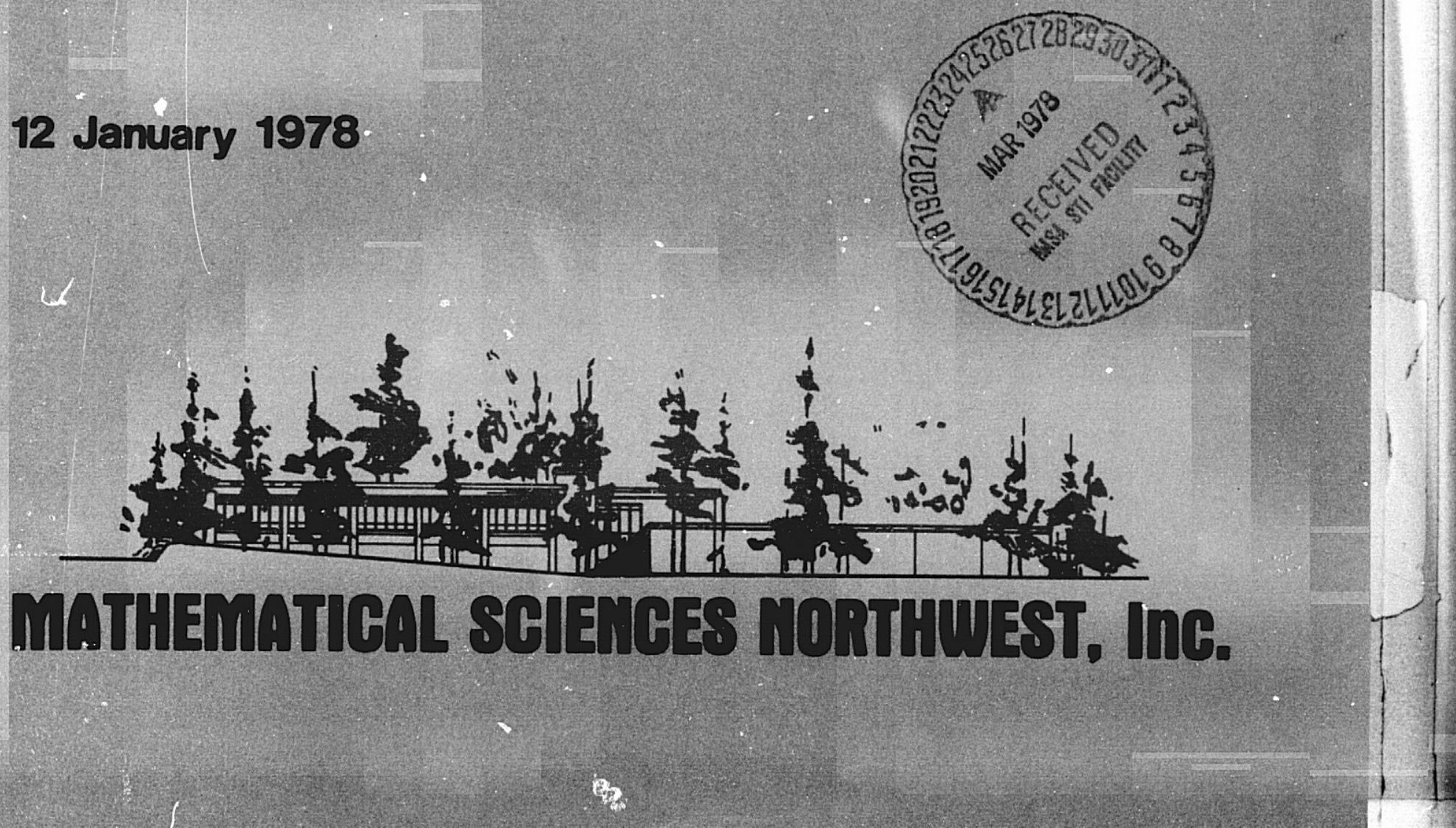
STUDY, OPTIMIZATION, AND DESIGN OF A

\section{LASER HEAT ENGINE}

FINAL REPORT

to

NASA-AMES RESEARCH CENTER

Moffett Field, California 94035

January 12,1978

by

MATHEMATICA SCIENCES NORTHWEST, INC.

P.0. Box 1887

Bellevue, Washington 98009 


\section{ACKNOWLEDGEMENTS}

This work was supported by the NASA-Ames Research Center, Moffett Field, California under Contract No. NAS2-9668. The work was carried out by the staff of Mathematical Sciences Northwest, Inc. (MSNW) of Bellevue, Washington under the direction of Dr. Robert Taussig. Other project staff included Drs. Phitip Cassady, Leonard NeIson, and Uennis Lowenthal, and Messrs. John Zumdieck, Stan Niece, Albert Pindroh, David Quimby, and Jim Lockett. MSNW consultants contributing to this project included Professors Gordon Oates, Reiner Decher, and Abraham Hertzberg of the University of Washington.

The authors express their gratitude to Dr. George Lee, NASAAmes Technical Contract Officer, and to Dr. Kenneth Billman of the NASA-Ames Research staff for their help and encouragement during the course of this project, and to the numerous groups contacted for technical information related to this project. 
Laser heat engine concepts, proposed for satellite applications, were analyzed to determine which engine concepts best meet the requirements of high efficiency (50 percent or better) continuous operation in space. The analys is of laser heat engines includes the thermodynamic cycles, engine design, laser power sources, collector/concentrator optics, receiving windows, absorbers, working fluids, electricity sneration, and heat rejection. We have concentrated on internally sated laser engine concepts, including piston engines, turbines ass 1 . . ad by energy exchangers, and direct energy converters, such as MHD generators. A comparison of thermal cycles was carried out in the context of these three main classes of engines to determine the most efficient concept within the bourds of available, near-term technology, and to suggest which engirie types have the most promise with further engineering development. In addition, suitable absorber-working fluid combinations for use with either $\mathrm{CO}$ or $\mathrm{CO}_{2}$ lasers were studied to determine upper temperature limits to heating, long-term chemical stability, reradiation losses, and the kinetics of absorption and thermal deactivation.

The best laser heat engine for a near-term experimental demonstration, selected on the bas is of high overall operating efficiency, nigh power-to-weight characteristics, and avallability of the required technology, is an otto/Diesel cycle piston engine using a diamond window to admit $\mathrm{CO}_{2}$ laser radiation. In closed cycle operation, the piston engine would use a helium working fluid doped with $\mathrm{D}_{2} \mathrm{O}$ (heavy water) as an infrared absorber. Theoretical calcuiations indicate that a., 0tto cycle thermal efficiency of approximately 54 percent may be achieved with a compression ratio of 6 . If the laser pulse length can be extended appropriately, then a Diesel cycle may be used with somewhat higher efficiency. The largest piston engine power which can be supplied by existing $\mathrm{CO}_{2}$ lasers available for non-military research is approximately one kilowatt. It appears possible to design and build this engine entirely on the basis of existing technology.

An experimental program is proposed with the objective of demonstrating laser heat engine operating efficiencies of 40 percent or greater during the first eighteen months of the program, and of reaching the 50 percent goal with further optimization. The proposed demonstration program would include a detailed engineering analysis of the engine design, engineering tests and development of the absorber and window components, construction and 
assembly of the complete engine, motoring tests (no laser heating), and performance tests (with laser heating).

The technology with the greatest promise of scaling to megawatt power leve?s in the long term is the energy exchanger/gas turbine combination. Appropriate turbine technology exists today, with good component efficiencies down to kilowatt power levels; however, these components cannot be used at the elevated temperatures required for a high efficiency laser heat engine. To circumvent these temperature limitations, a compact work transmission device called an energy exchanger can be used to transfer the work from the high temperature laser heated gases to low temperature gases to drive the turbine. Energy exchangers have been built in both small and large sizes for high temperature operation, but have not been optimized for high component efficiency. The development of a high efficiency energy exchanger is currently under consideration by the Department of Energy, and in the near future may provide a viable al ternative to the laser heated piston engines proposed for the near term demonstration. 
TABLE OF CONTENTS

SECTION

PAGE

I

INTRODUCTION

丁

II LASER HEAT ENGINE CONCEPTS 5

2.1. Thermodynamic Cycle Analysis 6

$\begin{array}{ll}\text { 2.2. Engine Concepts } & 16\end{array}$

2.2.1. Energy Exchanger/Turbine 17

2.2.2. Piston Engines 31

2.2.3. MHD Power Generator 45

2.3. Laser Power Sources $\quad 57$

2.3.1. Wavelength Characteristics 57

2.3.2. Modes of Operation 58

2.4. Collector/Concentrator 60

2.4.1. Transmitter and Receiver Mirror Dimensions 63

2.4.2. Receiver: Optical Design 65

2.4.3. Single Element Collectors 66

2.5. Receiving Windows 70

2.5.1. Double Window Configuration 71

2.5.2. Thermal Stresses 72

$\begin{array}{ll}\text { 2.5.3. Pressure Stresses } & 74\end{array}$

2.6. Radiation Absorbers and Working Fluids 75

2.6.1. Absorption Mechanisms and Working Fluids 75 
2.6.2. Absorption of Radiation from CO Lasers

2.6.3. Absorption of Radiation from $\mathrm{CO}_{2}$ Lasers

93

2.7. Mechanical-Electricat Conversion

2.8. Heat Rejection

2.8.1. Specific Radiator Area

2.8.2. Material Limits

101

II EVALUATION OF LASER HEAT ENGINE TECHNOLLGY

104

3.1. Criteria for Optimum Design

104

3.2. Mission Constraints

106

3.3. Selection Priorities

109

3.4. Near-Term Technology

110

3.5. Efficiency

111

3.6. Scaling to Larger Powers

114

3.6.1. Efficiency-Power Dependence 114

3.6.2. Size-Power Dependence

3.7. Summary of Evaluation

IV THE DEMONSTRATION EXPERIMENT

4.1. Description of the Baseline Laser Heat Engine System

4.1.1. Cycle Characteristics

4.1.2. Lubrication

4.1.3. Piston Sealing

4.1.4. Window and Block Cooling 
4.1.5. Piston Heat Transfer 128

4.2. Engine Operation 128

4.3. Experimental Program 129

4.4. Site and Facility Requirements 136

4.5. Longer Range Laser Heat Engine Program 137

4.5.1. Brayton/Energy Exchanger/CO Cyle 138

4.5.2. Systems Implication of Laser Heat Engines 138

$V \quad$ SUMMARY OF RECDMMENDATIONS AND CONCLUSIONS 140

5.1. Near-Term Demonstration Program 140

5.2. Development Program for Large-Scale Laser Heat Engines $\quad 140$

$\begin{array}{ll}\text { REFERENCES } & 142\end{array}$ 


\section{LIST OF FIGURES}

FIGURE

PAGE

1 Collector/Concentrator and Waste Heat Radiator for a Laser Heat Engine Mounted on a Standardized Satellite

$2 \quad$ Engine Cycles

3 Engine Cycles 7

4 Hybrid Carnot Cycles 8

5 Cycle Comparison for Fixed Maximum and Minimum Cycle Temperatures and Minimum Pressure

6 Comparison of Maximum Work Diese], 0tto, and Brayton Cycles with GP and Stirling Cycles

7 Schematic of Energy Exchanger/Turbine Laser Heat Engine

B $\quad x$-t Diagram for an Ideal Energy Exchanger Cycle

9 Cutaway View of Energy Exchanger with Rotating Tubes

10 Isometric View of CAL Wave Superheater Used to Provide High Temperature Air for Re-entry Simulation

il Photograph of the Ful1-Scale Gasdynamic Wave Heater Drum Constructed and Operated at CAL

12 Schematic of the Supercharging Application of the Comprex Wave Superheater Currently Being Marketed by Brown-Boveri Company

13 Schematic of Intercooled, Regenerated Brayton Cycle Utilizing an Energy Exchanger for High Efficiency Operation

14 Energy Exchanger/Turbine Efficiency and Regenerator Peak Temperature $\left(T_{5} / T_{1}\right)$ as Functions of Maximum-toMinimum Temperature Ratio and Compressor Ratio 
15 Schematic for a Piston Laser Heat Engine 32

16 Comparison of Combustion $(\gamma=1.273)$ and Monatomic Gas $(\gamma=5 / 3)$ Otto Cycles

17 Effects of Heat Transfer on 0tto Cycle Efficiency for Different Peak Temperatures and Working Fiuids

18 Stirling Engine Test Data for Operating Efficiency Engines

19 Stirling Cycle with Laser Heat Addition

20 Temperature Nonuniformities in a Laser Heated MHD Channe]

21 Geometrical Arrangement for Staged Laser Heating of an MHD Channel

22 Staged Transverse Converter Using Solid Absorption Surfaces

23 Mol]ier Diagram for Staged MHD Generator

24 (a) Cross-Section of MHD Duct with Heating Vanes and Nozzle, and (b) Stagnation and Fluid Temperatures versus Axial Distance

25 Variation of Maximum Load Factor with Flow Mach Number for Fixed $B(=2)$ and $T_{e} / T_{t}$

26 MHD Generator Efficiency vs. Stage Temperature Ratio for $N$ Stages

27 Specific Work vs. Stage Temperature Ratio for a Regenerated Multi-Stage MHD Generator

28 Specific Work vs. Stage Temperature Ratio for MHD Generator with No Regeneration

29 Transmitter Mirror Diameter, $D_{t}$, versus Receiver Mirror Diameter, $D_{C}$, for Various Wavelengths and Ranges 
30 Mirror Deformation and Pointing Error Limits as

Functions of Focal Length and Window Diameters

69

31 Face-Cooled Double Window Mounting Configuration 71

32 Output Spectra of Typical Co Lasers 79

33 Minimum Radiator Area, Brayton Cycle 100

34 Radiator Fin and Tube Relative Weight for a TurboGenerator Rankine Cycle

35 Laser Heat Engine System with Major Components and Efficiencies

36 Scaling of Prime Mover Efficiency witn Power 115

37 Baseline Laser Heat Engine Systen 121

38 Tentative Schedule for Laser Heat Engine Experimental Demonstration 


\section{LIST OF TABLES}

TABLE

1 Existing Combinations of Engine Concepts and Thermal Cycles

2 Summary of Cycle Efficiency and Work Ratio 9

3 Comparison of Maximum Work Cycles for $T_{3} / T_{1}=10 \quad 15$

4 Distribution and Availability of Heat Loss 35

5 Distribution of Availability Losses 38

6 Comparison of $r=12$ to $r=6.2$ for a Monatomic Gas Otto Cycle Engine

7 Existing High Power $\mathrm{CO}_{2}$ Lasers 61

8 Existing High Power CO and HF/DF Lasers 62

9 Relevant Parameters for Low Earth and Geostationary Orbits

10 Mechanical and Thermal Properties of Various Window Materials

11 Temporal Decomposition of N0 83

$12{ }^{12} \mathrm{C}^{16} \mathrm{O}$ Laser and ${ }^{13} \mathrm{C}^{18} 0$ Absorber Lines 84

13 Thermodynamic State of $98 \mathrm{Ar} / 1 \mathrm{CO} / 1 \mathrm{He}$ Gas 90

14 Summed Absorption Coefficient 9

15 Material Properties 102

16 Summary of Evaluation 116

17 Laser Heat Engine Baseline Design 121

18 Properties of Natural Diamonds. 123 
$\begin{array}{ll}\text { TABLE } & \text { PAGE }\end{array}$

19 Eesign Options for Engine Lubrication 124

20 Sorne Carbon Wear Rate Data 126 


\section{INTRODUCTION}

In 1973 and 1975, NASA-sponsored conferences on Laser Energy Convarsion [1, 2] were held at the Ames Research Center encompassing to breadth of laser conversion concepts from pure inverse absorption non-thermal engines to chemical processes, plasma thermionic diodes, and heat engines. of all these, only the heat engine showed distinct promise of achieving an overall energy conversion efficiency, from laser energy in to electric energy out, in excess of 50 percent.

Based on this initial appraisal, a study comparing laser heat engine concepts, their optimum configurations, and a design of the best laser heat engine for a near-term experimental demonstration was carried out by Mathematical Sciences Northwest, Inc. (MSNW) under NASA-sponsorship. The results of that study are summarized in this report.

Within the heat engine category, there are two distinct types of converters: internally and externally heated engines. Externally heated engines universally suffer from material temperature restrictions which limit the level of efficiencies achievable. That is, heat must be conducted through the outer engine wall or heat exchanger to heat the working fluid. In contrast, for internaliy heated engines, laser radiation can be absorbed directly through a window, without the severe temperature limits on thermal efficiency. Our studies have therefore concentrated on internally heated laser engine concepts, including piston engines, turbines assisted by energy exchangers, and direct energy converters, such as MHD generators.

The basic components common to all such engines are collector/ concentratcr; for receiving the laser radiation; a high strength, transparent laser radiation window; a radiation absorbing, high temperature working fluid exposed by the window to the laser radiation and contained by a high pressure cell; an expansion chamber for converting thermal energy to work energy; and, a mechanical device for transferring power from the expansion chamber to an electrical generator. A radiator is also required to reject waste heat from engine operation. A sketch of a sateilite with a laser heat engine is shown in Figure 1.

A variety of thermodynamic cycles can be employed, including Diesel, Otto, Brayton, Stirling, and other cycles for the heat engines mentioned above. An extensive comparison of thermal cycles was carried out in the context of the three main classes of engines 


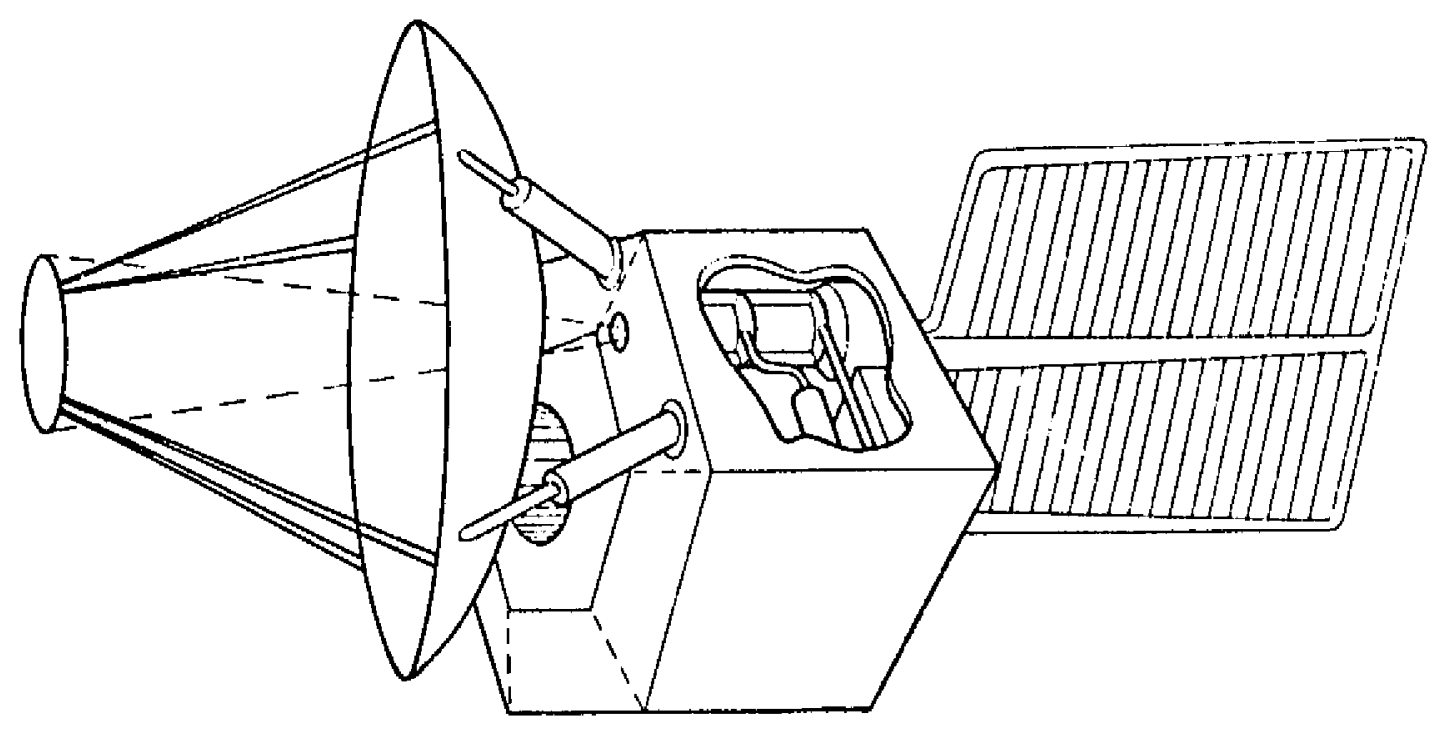

7701262

Figure 1. Collector/Concentrator and Waste Heat Radiator for a Laser Heat Engine Mounted on a Standardized Satellite

types (e.g., piston, turbine, and NHD) to determine the most efficient concept for a near-term denonstration, and to suggest which engine types have the most promise with further engineering development. Suitable absorber-working fluid combinations for use with either $\mathrm{CO}$ or $\mathrm{CO}_{2}$ lasers were studied to determine upper temperature limits to huating, long-term chenical stability, re-radiation losses, and the kinetics of absorption and thermal deactivation.

The best laser heat engine for a near-term experimental demonstration was selected on the basis of potential for high overall operating efficiency, high power-to-weight characteristics, and availability of the required technology. These selection criteria were supplenented by a preliminary consideration of mission constraints deduced from literature on space vehicle applications anticipated for the next few decades. Ranges for the power output, engine size, orbit location, and competitive power systens were taken from that literature. Information on the current status of technology was obtained froin a wide group of laser, engine, and parts manufacturers. In general, it was possible to design the experimental denonstration engine entirely on the bas is of existing technology. 
Both private and government sites for the proposed demonstration experiment were surveyed, with a particular emphas is on identifying and characterizing appropriate laser facilities. Other considerations included the availability of scientific staff, technicians, test equipment, and local contractors capable of constructing and operating the facility. Availability of the laser appears to be the pacing item in the recommendation of appropriate sites.

A longer-term program for laser heat engine development was also considered to overcome some of the problens of scaling this class of devices to larger sizes and to extend the limits on the overall efficiency of the near-term demonstration.

The near-term experimental demonstration engine selected is an 0tto/Diesel cycle piston engine using a diamond window to admit $\mathrm{CO}_{2}$ laser radiation. In closed cycle operation, the piston engine would use a helium working fluid doped with $D_{2}{ }^{0}$ (heavy water) as an infrared absorber. To avoid fouling of the window and heat exchanger components, the piston would be dry lubricated at the cylinder walls. Theoretical calculations indicate that thermal efficiency of approximately 60 percent may be achieved with a compression ratio of 6 . A Diesel cycle can be used with somewhat higher efficiency if the laser pulse length can be extended appropriately.

An eighteen-month demonstration program is being proposed to test the basic concepts of laser light absorption and heating and to establish the practical limits of operating efficiency. Absorption cell experiments, motoring (no laser heat addition) experiments, single pulse absorption, and open cycle experiments are outlined.

A development program for an engine based on a co laser, absorbing at five microns through window materials transparent to co laser radiation, is also proposed. This can be a relatively low cost addition to the basic demonstration program with potentially significant dividends in terms of device efficiency, and is within the state-of-the-art of laser technology.

The technology with the greatest promise of scaling to higher power levels in the longer-term is the energy exchanger/ gas turbine combination. Appropriate turbine technology exists today, with good component efficiencies down to kilowatt power levels; however, these components are limited to inlet temperatures on the order of $1200^{\circ} \mathrm{K}$ and cannot be used at the elevated temperatures required for a high efficiency laser heat engine. To circumvent this inlet temperature limitation, an energy exchanger [3], a compact work-transmission device based on an unsteady, gas piston 
compression-expansion cycle, can be used to transfer the work from the high temperature laser-heated gases to a low temperature gas to drive the turbine. Energy exchangers have been built in both small and large sizes for high temperature operation, but have not been optimized for high component efficiency. Development is necessary to raise the component efficiency of this device to desired levels. That development is currently under consideration by the Department of Energy, and in the near future may provide a viable alternative to the laser heated piston engines proposed for the near-tern demonstration which are best suited to, at most, the several hundred kilowatt power levels. Such engines would be based on a co laser with $\mathrm{CO}$ as an absorber, and would require a laser whose spectral output is tailored to the absorbing medium. Such lasers are currently within the state-of-the-art. 


\section{LASER HEAT ENGINE CONCEPTS}

A variety of laser energy conversion concepts have been studied, but only a few have shown a potential for achieving high energy conversion efficiencies, i.e., above 50 percent [4-9]. These high efficiency concepts are heat engines, such as piston engines and turbines, and direct energy converters, such as magnetohydrodynamic (HHO) generators.

Each of these engines can employ one of several different types of thermodynamic cycles, depending upon its mode of operation. For example, the piston engine has been used with the otto cycle, the Diesel cycie, and the Stirling cycle. The turbine engine has been used primarily with the Brayton cycle and the Rankine cycle; with multiple reheat and intercooling stages, the turbine Brayton cycle approaches the Ericsson cycle. A summary of the main engine types and thermodynamic cycles is shown in Table 1 . The $X$ 's indicate combinations of engine and cycle which have been designed and operated.

Table 1

Existing Combinations of Engine Concepts and Thermal cycles

\begin{tabular}{|c|c|c|c|}
\hline \multirow[b]{2}{*}{ THERMAL CYCLES } & \multicolumn{3}{|c|}{ ENGINE CONCEPTS } \\
\hline & $\begin{array}{c}\text { ENERGY EXCHANGER } \\
\text { TURB INES }\end{array}$ & $\begin{array}{l}\text { PISTON } \\
\text { ENGIINE }\end{array}$ & $\begin{array}{c}\text { MHD } \\
\text { GENERATOR }\end{array}$ \\
\hline Rankine & $x$ & $x$ & \\
\hline Brayton & $x$ & & $x$ \\
\hline ot to & & $x$ & \\
\hline [iesel & $x$ & $x$ & \\
\hline Ericsson & $x$ & & $x$ \\
\hline Stirling & & $x$ & \\
\hline Hybrid Cycles & $x$ & & \\
\hline
\end{tabular}


The mechanism of heat addition and heat rejection determine the particular thermodynamic cycle used with a given type of engine. For instance, in a spark ignition piston engine, the otto cycle is used because of the rapid, nearly constant volume heat addition resulting from a spark-ignited combustion process. Similarly, by opening the exhaust valve to ambient pressure, a constant volume expulsion of hot exhaust gases is achieved after most of the available work has been extracted. Clearly, the manner in which laser energy can be absorbed in the working fluid and the requirements of closedcycle operation with heat rejection to outer space will also affect the particular cycle choice for each engine type.

The following sections deal with an investigation of themodynamic cycles appropriate to laser heat addition and space heat rejection for each of the major engine types identified above. The thermal and mechanical efficiencies for each cycle/engine combination are developed, and the variation of these efficiencies with operating temperatures, pressures, and working fluid thermal properties are discussed. In order to understand the range of improvement possible with increasing size, approximate scaling laws have been prepared. These relationships, along with engineering constraints, mission objectives, and availability of technology, have been used to help select the most promising laser heat engine concept.

Because of the remote application (e.g., in a space satellite) for laser heat engines, it is also desirable to determine the laser energy receiver components, mechanical-to-electrical conversion components, and heat rejectior components appropriate to these heat engine concepts. The following sections also include a discussion of these topics.

\subsection{Thermodynamic Cycle Analysis}

We have investigated six conventional thermodynamic cycles and several hybrid Carnot cycles for appiication to laser heat engines. The $p v$ and Ts diagrams are illustrated in Figures 2, 3, and 4. The ideal Carnot cycle is also shown for comparison in Figure 3. Algebraic forms for the ideal thermal efficiency and the work ratio, defined as

$$
\eta=\frac{\text { net work out }}{\text { heat in }}
$$

and

$$
r_{w}=\frac{\text { work out }}{\text { work in }}
$$



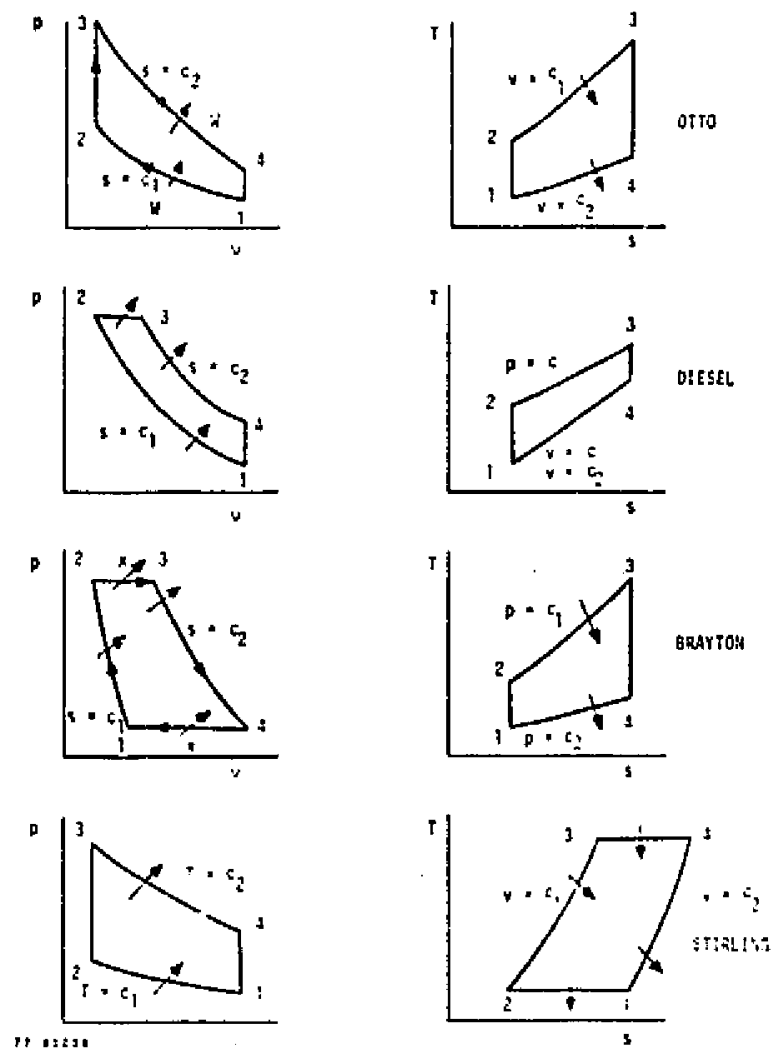

Figure 2. Engine Cycles
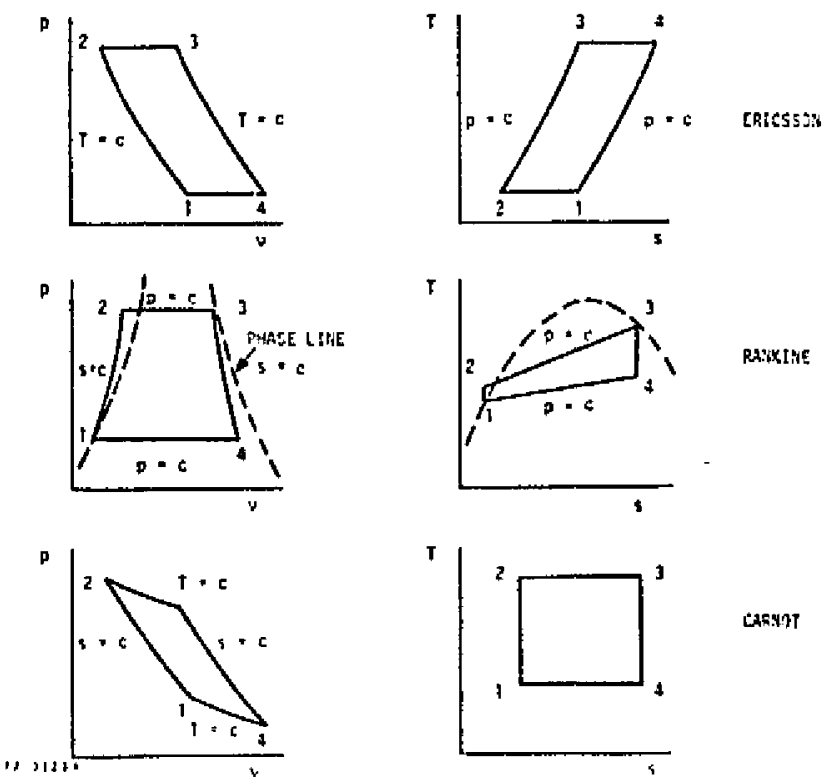

Figure 3. Engine Cycles 
(1)

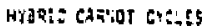
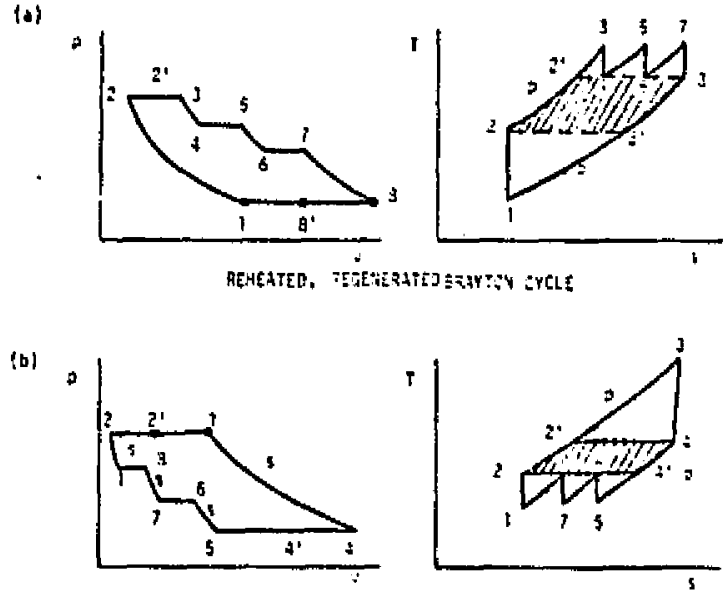

IMTERCOOLED, AEGEAERATEO BAST TOY CTELE

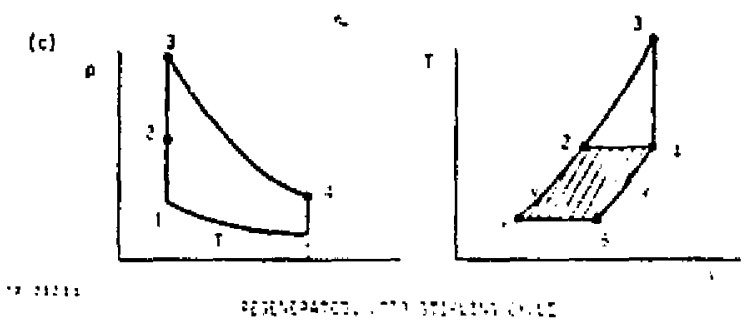

figure 4. Hybrid Carnot Cycles

have been derived for each of these cycles and are summarized in Table 2 (subscripts correspond to the points labeled in Figs. 2. 3 , and 4).

The hybrid Carnot cycles are variants of the Brayton and Stirling cycles, which allow an improvement over the simpler versions of these cycles. In the case of the Brayton cycle, intercooling, reheating, and regeneration allow the theoretical cycle efficiency to approach that of the Ericsson cycle, which has the same efficiency as the Carnot cycle. The regenerated 0tto/Stirling cycle permits a peak gas temperature in excess of the highest material temperature in the engine, and hence elevates the ideal themal efficiency above the bounds set by wall materials on the Stirling cycle efficiency. This only appears to be feasible for internal gas heating, such as the laser can provide. The GP cycle, developed by Garbuny and Pechersky, resembles one-half of the Stirling cycle, and takes advantage of constant volume heating with isothemal heat rejection [5].

It is possible at the outset to eliminate one of these cycles from consideration for laser heat engines. The Rankine cycle has been studied for space heat engines and compared to Brayton and Stirling engines $[10,11]$, down to powers on the order of 
Table 2

Summary of Cycle Efficiency and Work Ratio

\begin{tabular}{|c|c|c|}
\hline CYCLE & IDEAL THERMAL EFFICIEICY ( & 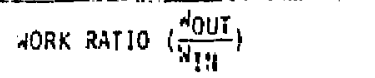 \\
\hline otto & $\begin{array}{l}\eta=1-\frac{1}{r^{r-1}} \\
\left(r=v_{1} / v_{2}\right)\end{array}$ & $r_{v}=\frac{T}{r_{1}}$ \\
\hline Olesel & $\begin{array}{l}n=1-\frac{\left\langle r_{p}^{r}-1\right)}{\left\langle r^{r-1}\left(r_{p}-1\right)\right.} \\
\left(r_{p}=v_{3} / v_{2}\right)\end{array}$ & $r_{w}=\frac{(i-1)\left(T_{3}-T_{2}\right)+\left(T_{3}-i_{2}\right)}{T_{2}-T_{1}}$ \\
\hline Brayton & $n=1-\frac{1}{r-1}$ & $r_{W}=\frac{T_{3}-T_{2}+\frac{1}{T-1}\left(T_{3}-T_{2}\right)}{T_{4}-T_{1}+\frac{1}{T=1}\left(T_{2}-T_{1}\right)}$ \\
\hline Stirling & $n=1-\frac{T_{1}}{T_{4}}$ & $r_{w}=\frac{T_{4}}{T_{1}}$ \\
\hline Ericsson & $n=1-\frac{T_{1}}{T_{4}}$ & $r_{w}=\frac{T_{1}}{T_{1}}$ \\
\hline Rankine & $n=\frac{h_{3}-h_{4}-\left(h_{2}-h_{1}\right)}{h_{3}-h_{2}}$ & $r_{w}=\frac{n_{3}-n_{2}}{n_{4}-n_{1}}$ \\
\hline \multicolumn{3}{|c|}{ HYERTO CARNOT CYCLES } \\
\hline $\begin{array}{l}\text { Rephejted, } \\
\text { Rejenerated } \\
\text { Bryyton }\end{array}$ & $n=1-\frac{2^{-1}}{3\left(T_{j}-T_{B}\right)}$ & 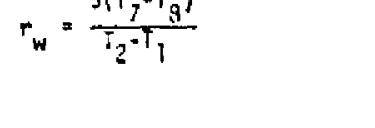 \\
\hline $\begin{array}{l}\text { intercooled, } \\
\text { Aegengurinted } \\
\text { Brayton }\end{array}$ & $n=1-\frac{3\left(T_{2}-T_{1}\right)}{T_{3}-T_{4}}$ & $r_{w}=\frac{T_{3}-T_{4}}{3\left(r_{2}-T_{1}\right)}$ \\
\hline 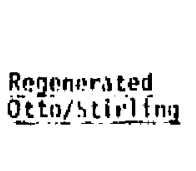 & $n=1-\frac{\mathrm{RT}_{1} \ln \left(\frac{v_{5}}{v_{1}}\right)}{c_{v}\left(r_{3}-v_{2}\right)}$ & $r_{W}=\frac{c_{p}\left(T_{1}-T_{2}\right)}{R_{1} \operatorname{Tm}\left(\frac{T_{v_{1}}}{v_{1}}\right)}$ \\
\hline 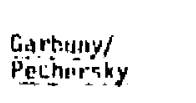 & 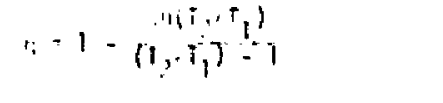 & $\because=1 \div$ \\
\hline
\end{tabular}


several kilowatts. In this size range, the material corrosion problems associated with the working fluids (i.e., a]kali metals or mercury) and complex behavior of two-phase fluids in a zero-gravity environment have consistently caused the Rankine cycle engines to be rated below the other two engine types. Thus, we shall leave Rankine cycles out of our comparisons.

Our theoretical comparison of thermodynamic cycles is 1 imited to the simple forms of these cycles, including the regenerated Brayton and Stirling cycles. The Ericsson cycle will be considered equivalent to the Stirling cycle, since the ideal thermal efficiencies are the same for these two cycles. The initial comparison will be made purely on the basis of thermal efficiency. Later on, when scaling to a larger size is considered and where the optimum efficiency must be determined, the work ratio information given in Table 2 will be used.

We have identified four types of comparisons which have a bearing on the space application of laser heat engines. Each of these consists in holding an appropriate number of the cycle parameters fixed, and then comparing the efficiencies, net work out, work per unit mass, and other figures of merit for each cycle. These are:

1) Fix the maximum and minimum cycle temperatures, the temperature at the end of the power stroke (or gasdynamic expansion process), and the maximum cycie pressure.

2) Fix the maximum and minimum cycle temperatures, the heat input per cycle, and the maximum cycie specific volume.

3) Fix the thermal efficiency, the heat input per cycle, the minimum cycle temperature, and the maximum cycle specific volume.

4) Fix the maximum and minimum cycle temperatures, the maximum gas volume, and the minimum gas pressure.

Each of these bases for comparison reflects the kind of constraints which could be placed on engine operation by materials, engineering design, and mission objectives. For example, cases 1, 2, and 4 constrain the peak and minimum cycle temperatures. One would choose these temperatures to be as far apart as possible so as to maximize the Carnot efficiency limit on the heat engine. It is worthwhile noting here that the real temperature cannot exceed that which can be obtained by the laser heating technique employed (e.g., resonant 
absorption heating). At the other extreme, the minimum cycle temperature cannot be too low or the size of the heat radiator will become too large. The other variables specified in cases 1, 2, and 4 have been chosen to jllustrate the type of constraint that heat rejection to a bottoming cycle would impose (i.e., the temperature at the end of the power stroke), that the limits of laser energy per pulse would impose (i.e., the heat input per cycle), or that the system size, weight, and working fluid power density would impose (i.e., maximum gas volume and minimum gas pressure). The type 3 comparison sets a premium on efficiency and allows the computation of peak cycle temperature as a figure of merit for each cycle. Excessive cycle temperatures raise a design and materials problem, as we 11 as enhancing thermal losses in the cycle at temperatures above the design waste heat rejection temperature.

The principal figures of merit for comparing different cycles are suggested by the need to achieve reliable operation in a space environment for long-time duration missions with greater than 50 percent overali efficiencies. Hence, high thermodynamic efficiency is essential, preferably in excess of 75 percent, in order to offset mechanical and electrical conversion losses in the system. Power per unit mass and unit volume are also significant measures of engine attractiveness, since both variables directly affect mission cost. Peak gas pressure is also important, since higher pressures will require heavier containing walls and windows, adding to the launch weight and inertia of the engine. An initial comparison of thermodynamic cycles will be carried out which considers the following measures:

- Ideal thermal efficiency

- Power-to-weight ratio

- Power-to-volume ratio

- Peak cycle pressure

We have chosen to use the fourth type of comparison, discussed previously in this section, because it offers an opportunity to optimize the work output for several of the cycles for approximately the same engine size and weight. The efficiency is welldefined for each of these cycles in terms of the work output optimization, and each of the other three parameters (e.g., power-toweight ratio, etc.) can be defined, too, once the efficiency has been determined.

Prescription of the maximum volume, minimum pressure, and minimum cycle temperature precisely determines a single point for 
the GP, Diesel, and 0tto cycles, but over-determines the Brayton cycle, since maximum cycle volume and minimum temperature are not achieved together at any single point in the Brayton cycle. Since the Brayton cycle is generally realized in a steady flow device, we relax the maximum volume requirement and assume that it has the same minimum cycle temperature as the other cycles. The peak cycle temperature is also prescribed for all of the.cycles. The optimization of net work output for each cycle follows:

Otto Cycle:

$$
\begin{aligned}
& \text { Net } W_{0}=c_{v}\left(T_{3}-T_{2}\right)-c_{v}\left(T_{4}-T_{1}\right) \\
& =c_{v} T_{3}(1-x)-c_{v} T_{1}\left(\frac{l}{x}-1\right) \\
& \text { (where } x=\frac{T_{1}}{T_{3}} r^{\gamma-1} \text { ) } \\
& \therefore \frac{\partial W_{0}}{\partial x}=0 \text { when } x=\sqrt{\left(T_{1} / T_{3}\right)} \text { or } r^{\gamma-1}=\left(\frac{T_{3}}{T_{1}}\right)^{1 / 2} \\
& * n_{0 \text { tto }}=1-\sqrt{\left(T_{1} / T_{3}\right)}
\end{aligned}
$$

Brayton Cycle:

$$
\begin{aligned}
& \text { Net } W_{0}=c_{p}\left(T_{3}-T_{2}\right)-c_{p}\left(T_{4}-T_{1}\right) \\
& =c_{p} T_{3}(1-x)-c_{p} T_{1}\left(\frac{1}{x}-1\right) \\
& \text { (where } x=\frac{T_{1}}{T_{3}}\left(\frac{p_{2}}{p_{1}}\right)^{\frac{\gamma-1}{\gamma}} \text { ) } \\
& \therefore \frac{\partial W_{0}}{\partial x}=0 \text { when } x=\sqrt{\left(T_{1} / T_{3}\right)} \text { or }\left(\frac{p_{2}}{p_{1}}\right)^{\frac{\gamma-1}{r}}=\sqrt{\left(T_{3} / T_{1}\right)} \\
& \therefore \quad n_{\text {Brayton }}=1-\sqrt{\left(\mathrm{T}_{1} / T_{3}\right)}
\end{aligned}
$$


Diesel Cycle:

$$
\begin{aligned}
& \text { Wet } W_{0}=c_{p}\left(T_{3}-T_{2}\right)-c_{v}\left(T_{4}-T_{1}\right) \\
& =c_{v} T_{3}\left\{\gamma(1-x)-\left(\frac{1}{x} \gamma-\alpha\right)\right\} \\
& \text { * } \frac{\partial W_{0}}{\partial x}=0 \text { when } x=\alpha^{\frac{1}{\gamma+2}} \text { or } r^{\gamma-1}=\left(\frac{T_{3}}{T_{1}}\right)^{\frac{\gamma+1}{\gamma+2}} \\
& \text { and } r_{c}=\left(\frac{T_{3}}{T_{1}}\right)^{\frac{1}{\gamma+2}} \\
& \therefore n_{\text {Diese } 1}=1-\left(\frac{T_{1}}{T_{3}}\right)^{\gamma+1}\left[\frac{\left(\frac{T_{3}}{T_{1}}\right)^{\frac{\gamma}{\gamma+2}}-1}{\left.\left(\frac{T_{3}}{T_{1}}\right)^{\frac{1}{\gamma+2}}-1\right\}}\right]
\end{aligned}
$$

The GP and Stirling cycles require no additional constraints, and their efficiencies are given by

$$
\begin{aligned}
& n_{G P}=1-\frac{\ln \left(T_{3} / T_{1}\right)}{\left(T_{3} / T_{1}\right)-\bar{T}} \\
& n_{\text {Stirling }}=1-\frac{T_{1}}{T_{3}}
\end{aligned}
$$

Figures $5 \mathrm{a}$ and $5 \mathrm{~b}$ show these optimized cycles in the pv and Ts planes, respectively. Figure 6 shows the efficiencies as a 


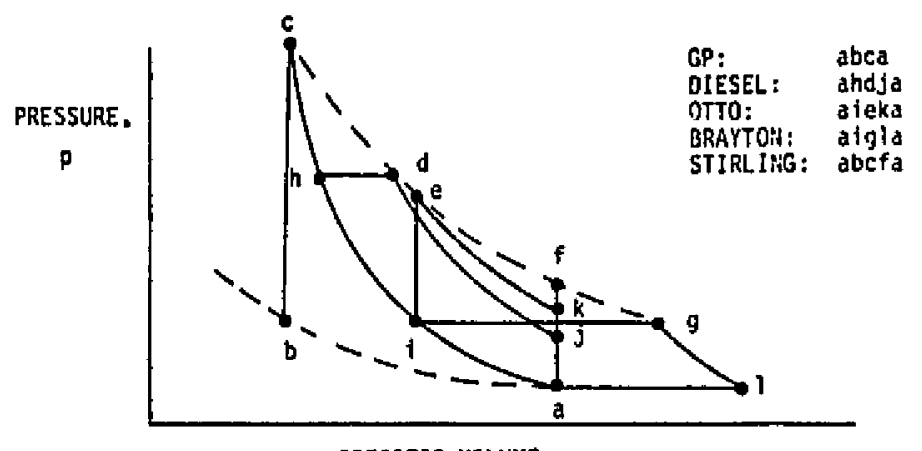

SPECIFIC VOLUME,

(a)

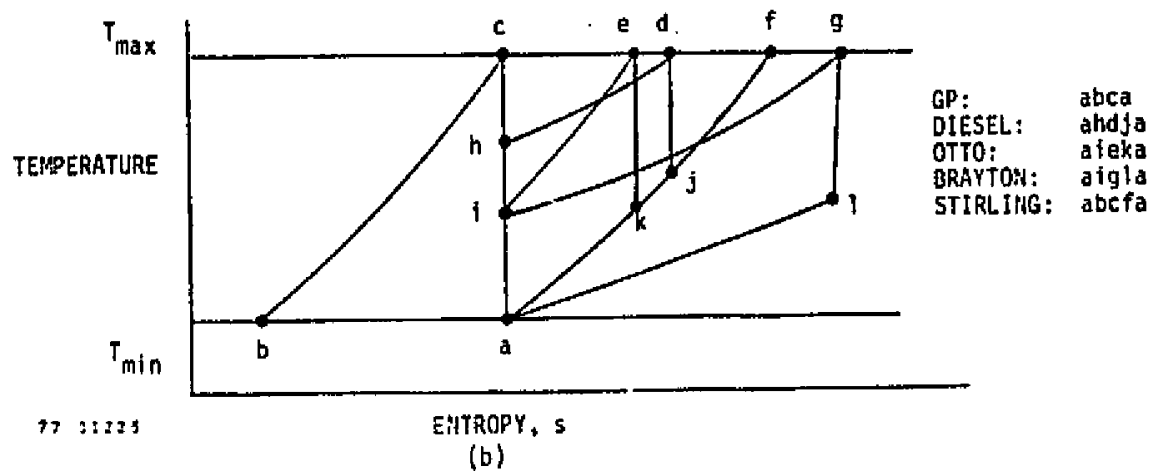

Figure 5. Cycle Comparison for Fixed Maximum and Minimum Cycie Temperatures and Hin imum Pressure

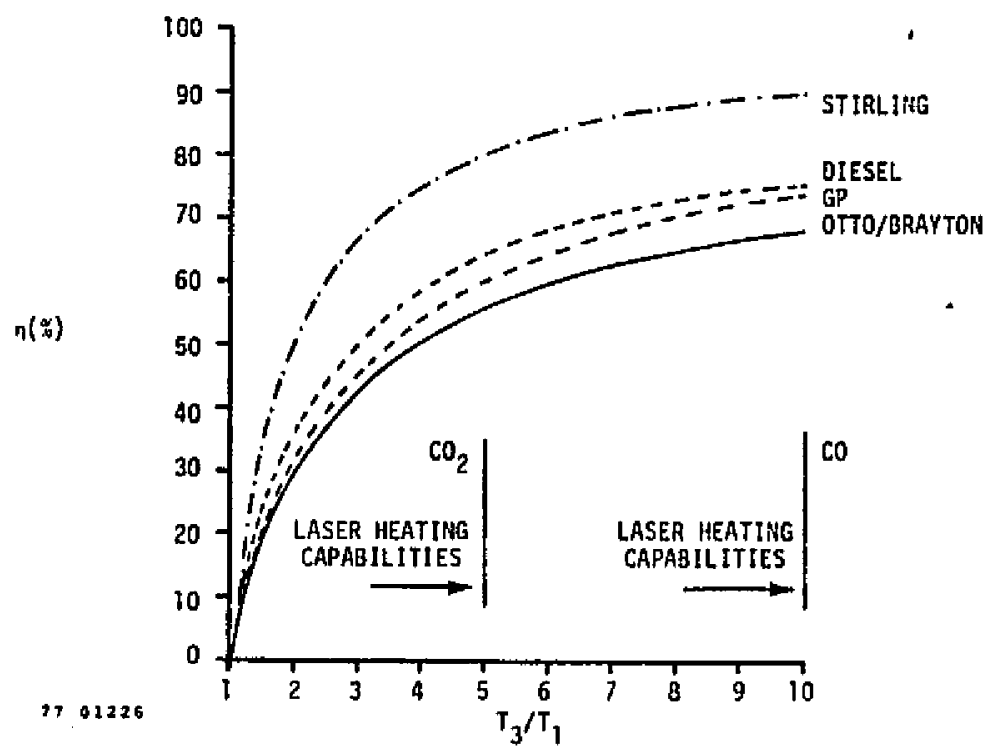

Figure 6. Comparison of Maximum Work Diesel, Otto, and Brayton Cycles with GP and Stirling Cycles (Minimum and Maximum Temperatures and Minimum Pressure HeTd Constant)

\section{ORIGINAL PAGE IS
OF POOR QUALITY}


function of $T_{3} / T_{1}$. Cleariy,

$$
n_{\text {Stirling }}>n_{\text {Diesel }}>n_{G P}>n_{\text {Otto }}=n_{\text {Brayton }}
$$

For temperatures $\left(T_{3} / T_{1}\right)>5$, it is a iso obvious that each of these cycles has a high ideal thermal efficiency. Since it appears feasible to achieve gas temperatures on the order of $3000^{\circ} \mathrm{K}$ by resonance absorption of laser radiation and to reject heat at temperatures on the order of $300^{\circ} \mathrm{K}$, temperature ratios $\left(T_{3} / T_{1}\right)$ up to 10 appear quite realistic. Therefore, other measures must be examined in order to sharpen this comparison and to allow a distinct choice of engine concepts. Table 3 lists the pressure ratio and intermediate temperatures required by each of these cycies, as well as the thermal efficiencies. For a one-atmosphere minimum cycle pressure, the GP cycle obviously requires extreme operating conditions (i.e., 316 atmospheres peak pressure). While not out of the question, this fact alone causes us to seek a lower pressure engine to reduce piston cylinder weight and laser window stresses, and to ameliorate seal losses of the working fluid. High compression engines are built routinely to withstand 1500 psia pressure. All of the other cycles lie within that constraint except the GP cycle. At this point, we eliminate the GP cycle from further consideration, especially since the Diesel cycle can attain even higher therma? efficiencies at lower peak pressures.

Table 3

Comparison of Maximum Work Cycles for $T_{3} / T_{1}=10$

\begin{tabular}{lccccc}
\hline & 0 TTO & DIESEL & BRAYTON & GP & STIRLING \\
\hline$\eta_{\text {Idea I }}$ & $68 \%$ & $76 \%$ & $68 \%$ & $74 \%$ & $90 \%$ \\
$\mathrm{P}_{3} / \mathrm{P}_{1}$ & 56 & 66 & 17.8 & 316 & 5.33 \\
$\mathrm{~T}_{2}$ & $949^{\circ} \mathrm{K}$ & $1600^{\circ} \mathrm{K}$ & $949^{\circ} \mathrm{K}$ & $3000^{\circ} \mathrm{K}$ & $3000^{\circ} \mathrm{K}$ \\
$\mathrm{T}_{4}$ & $949^{\circ} \mathrm{K}$ & $854^{\circ} \mathrm{K}$ & $949^{\circ} \mathrm{K}$ & $300^{\circ} \mathrm{K}$ & $300^{\circ} \mathrm{K}$ \\
\hline
\end{tabular}


In practice, it is difficult to distinguish the 0tto cycle from the Diesel cycle, since a continuous range of intermediate cycles exists in which the only variable is the timing of energy rel ease in the vicinity of top dead center. Thus, the three surviving cycles to be considered in greater detail are:

- Ctto/Diesel cycle

- Brayton cycle

- Stirling cycle

The major losses associated with each of these cycles witl be analyzed in order to get a more accurate measure of the actual thermal efficiency. That analys is is carried out in Section 2.2 for the specific engine types to which each of these cycles can be applied effectively. Also included in Section 2.2 is a discussion of scaling to Targe size which yiclds the specific power ratios for each engine.

\subsection{Engine Concepts}

High temperature operation of heat engines is required in order to attain high thermal efficiences for a given fixed heat rejection temperature. The temperature drop from peak temperature to heat rejection temperature can be taken in severa? stages, forming a combined cycle system, where the heat rejection temperature of one stage becomes the peak temperature of the next lower stage. This cascade of thermal cycles is largely necessitated by engineering considerations, for example, to avoid large thermal stresses in insulating walls and to avoid the flow losses which would occur if all of the work were taken out in a single stage (e.g., in a single turbine). Hence, combined cycle operation constitutes a natural form of high efficiency heat engines. Several different types of engines may be combined to extract the most work from the various stages. For instance, the topping cycle might use a piston engine or turbine engine with a high temperature exhaust to run a turbine engine bottoming cycle. In smaller engines, the added complications of additicnal thermal cycles usually negate the possibility of increased efficiency. In our considerations, combined cycle operation has been relegated to longer term laser heat engine development where scaling to larger sizes is warranted.

The following paragraphs describe several laser heat engine concepts, including their design and operation. Component efficiencies of the most promising engine/cycle combinations are presented and highi temperature design and materials constraints are outlined 
briefly. Because the working fluid may also act as the energy absorber, special attention is given to working fluids which perform both of these functions.

Three basic heat engine concepts have been investigated: energy exchanger/turbines, piston engines, and MHD channels. Schematics of these engines zre shown in Figures 7, 15, and 22 to illustrate some of the details of their design and operation. Their application to laser energy conversion is discussed below.

\subsubsection{Energy Exchanger/Turbine}

For all of the engines discussed here, we assume that the laser radiation is collected and focussed through a receiving window in a "radiation" cavity where it is volumetrically absorbed by the primary working fluid, heating it to a peak temperature, $T_{2}$. In some instances, the energy absorbing material is not the primary working fluid; this variation will be discussed separately later. In this particular engine concept, which is shown in Figure 7 , the hot exhaust gas from the absorption cavity is injected directly into an energy exchanger where it transfers its energy to a cooler, lower molecular weight, driven gas. This cooler gas serves as a secondary working fluid to drive the gas turbine. Because the driver and driven gas are in direct contact with each other in the energy exchanger and may intermix, a gas cleanup system may be necessary in order to maintain the proper molecular weight difference between these two gases. This cleanup stage may be located either on the driven gas loop or on the driver gas loop.

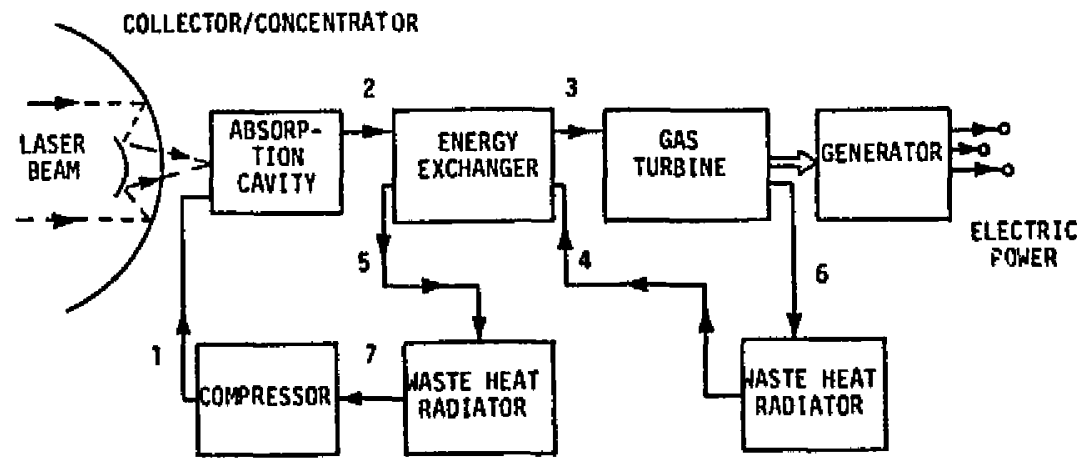

$n 0.1227$

Figure 7. Schematic of Energy Exchanger/Turbine Laser Heat Engine 
The driver gas at stage 5 may transfer more of its energy by heat conduction to a second closed-loop bottoming cycle before being recompressed at stage 6 for re-entry to the radiation boiler absorption cavity at stage 1. The bottoming cycle can be any closed cycle which operates efficiently between the temperatures $T_{5}$ and the low temperature limit imposed by the waste heat radiator. Under some circumstances, the driver fluid itself could be used as the bottoming cycle working fluid and the heat exchanger could be eliminated. Since the bottoming cycle is common to each of the engine concepts, it will be discussed separately later.

The major components in this engine are:

- Collector/concentrator and pointing controls

- Receiving window

- Radiation absorption cavity

- Energy exchanger*

- Topping cycle turbine*

- Gas separation stage*

- Compressor*

- Combined cycle heat exchanger (optional)

- Bottoming cycle (optional)

- Waste heat radiators

The starred items are specific to this engine concept and are discussed in more detail below. The other items, as indicated earlier, will be discussed separately later.

While all of the engine concepts have some form of radiation absorption cavity, some specific variations are important enough to warrant a description of the interaction of this cavity with individual engines. In the case of the energy oxchanger/turbine, the radiation cavity may be a separate component specifically designed to achieve high temperatures through the absorption of laser radiation. Limits to the temperature which can be achieved depend both on the ability of the working fluid to absorb radiation at high temperatures and on the heat losses from the cavity. 
Heat is lost from the cavity through the walls by heat conduction and radiation to space. Heat can also be lost by reradiation back through the receiving window. In some engines, the wall losses can be reduced by piping the incoming working fluid through the walls of the boiler so that the outer wall temperature of the boiler is kept low. Designs similar to this have been employed successfully in rocket engines to preheat the fuel and to avoid wa 11 damage from the high temperature exhaust gases. Gas temperatures as high as $3400^{\circ} \mathrm{K}$ have been achieved in continuous flow cavities with this type of design [12]. Losses from the window can be reduced by making the window smaller and reflective on the inside surface.

Heat is transferred from the working fluid to the cavity walls by conduction, forced convection, and radiation. Material heat transfer processes can be reduced by focussing the light at the cavity center away from the walls and window. Garbuny and Pechersky analyzed the feasibility of achieving localized heating in this way, and found that it was possible if the absorption coefficient is not much larger than the (absorption distance) ${ }^{-1}$ in the gas [5]. Minimizing the surface-to-volume ratio will also help to reduce conduction losses to the walls.

Forced convection heat transfer can be decreased by running the engine at low rpm or low flow speeds. However, these two approaches tend to require larger engines for the same power output.

Reradiation heat transfer from the working fluid/absorber gas to the cavity walls and window depends on the gas temperature and emissivity, and on the cavity wall and window temperature and reflectivity. The reradiated wavelengths will generally be quite different from the incident laser wavelength. The absorber and working fluid emission spectra at the peak cycle temperature must be integrated to obtain the proper emissivity. If helium or argon is used as the working fluid, then relatively little energy will be lost by radiation from that part of the mixture because the first excited states of these noble gases are at high energy levels. Tightly-bound, infrared laser absorbers, such as CO or NO, also have a small emissivity, but polyatomic absorbers, such as $\mathrm{SF}_{6}$ and $\mathrm{H}_{2} 0$, may readily reradiate energy. Unless the window and interior walls are reflective to the reradiated wavelengths, all of that energy will be lost and unavailable for doing work. Sample calculations will be presented for the evaluation of particular engine concepts in Section III.

Energy exchanger concepts have been developed which promise to increase substantially the thermal efficiency of turbine power generators. The energy exchanger is a compact device which transmits the work of expansion of a high temperature gas through a gas 
interface to a colder, lighter molecular weight gas which, in turn, drives a conventional turbine. Because this is a work transfer device, the overali thermal efficiency is determined by the high temperature of the driver working fluid and is independent of the tenperature of colder, driven gas [6].

Since the advent of shock tube technology, it has been known that gasdynamic compression processes are a flexible method of transferring energy from one gas to another, i.e., from a highpressure driver gas to a lower pressure driven gas. It can be shown that the transfer of energy across a gas interface by a compression wave is faci?itated by a condition called impedance matching, which requires that no acoustic wave be reflected from the interface, i.e., the original wave be transmitted in full strength. In terms of the state of the two gases, impedance matching means that the specific heat ratio, $\gamma$, and the product of the density and sound speed, pa, must be the same on each side of the interface. The combination of these two conditions guarantees that the ratio of the gas temperatures on either side of the interface is equal to the ratio of the molecular weights. That is, by choosing the molecular weight ratio of driver to driven gas (i.e., impedance matching), we can control within wide limits the temperature of the colder driven gas to be compatible with conventional turbine inlet temperature limits. Thus, a high efficiency thermal cycle can be designed using the energy exchanger to couple a high temperature source to conventional low temperature turbines.

Under ideal conditions of impedance matched gases and isentropic compression followed by isentropic expansion, the work transferred by the hot driver gas to the cold driven gases is a maximum, and the losses, in principle, can be zero. That is, a theoretical component efficiency of 100 percent is possible for the el.ergy exchanger. In actual operation, losses due to seal leakage, boundary layers, weak shock waves, and mixing at the interface may limit real component efficiencies to 85 or 90 percent [6].

Figure 8 shows the complete ideal gas compression-expansion cycle in a single tube of the energy exchanger. The horizontal axis refers to positions along the length of the tube. The tube is open at both ends and can be filled with driver gas and driven gas, separated by a narrow interface (dashed line in Fig. 8). The vertical axis indicates successive instants in time during one complete compression-expansion cycle of the energy exchanger. At any given time in this cycle, the solid and dashed 1 ines of Figure 8 show the instantaneous position of the expansion or compression waves and the gas interface in the tube. As time progresses, these waves and the gas interface move along the tube. This piston-like action transfers the work from the driver gas to the driven gas across the interface. 


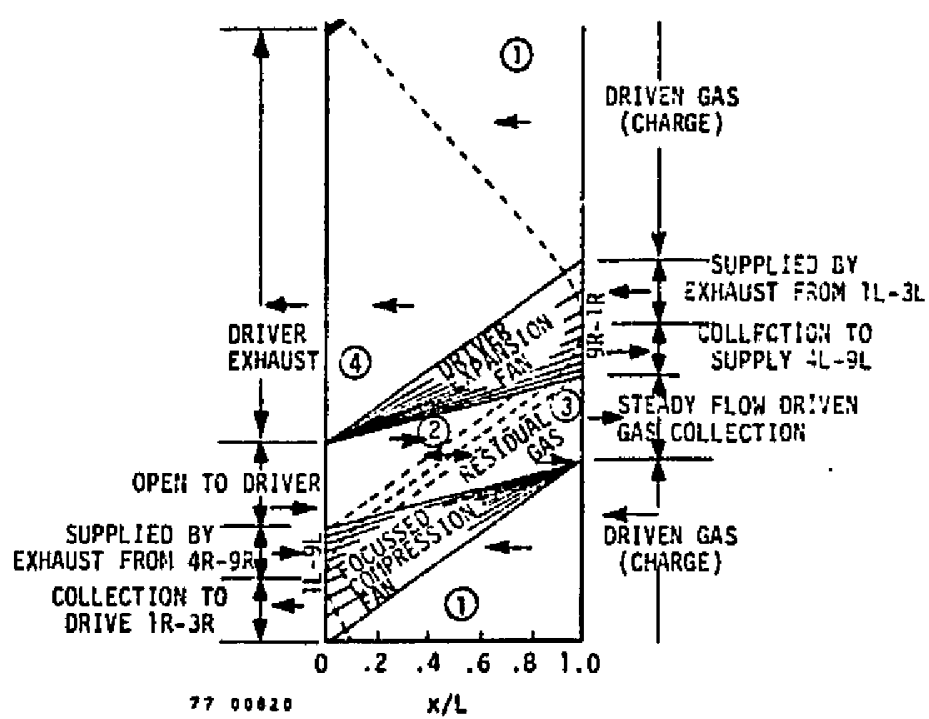

Smooth compression and expansion with no wave reflection from the ends of the energy exchanger tubes is accomplished by rerouting some of the driver and driven gases from one end of the energy exchanger to the other. The dashed jine shows the contact surface between the driver and driven gases. State 1 is the entrance condition of the driyen gas. This gas is compressed as close to isentropically as possible to the final discharge State 3. The compresston is accomplished by the driver gas entering in \$tate 2 ; the driver gas is then expanded to state 4 by opening the tube ends to a lower pressure. The process then repeats itself with driven gas charge in state 1. The temperature ratio between 5 tates 2 and 3 is directly proportional to the molecular weights: $T_{2} / T_{3}=m_{2} / m_{3}$.

Figure 8. x-t Diagram for an Ideal Energy Exchanger Cycle

In order to produce a steady flow process, multiple energy exchanger tubes are mounted on the rim of a rotating drum, such as shown in the configuration in Figure 9. As the drum rotates past a series of shaped nozzles and compression walls, the gasdynamic cycle is repeated consecutively in each tube, approaching a steady flow. High temperature materials problems are eliminated or mitigated to a large extent in the energy exchanger by the continual cycling of the low temperature driven gas through the tubes.

Energy exchanger devices have been built and operated for large, high temperature $\left(4500^{\circ} \mathrm{K}\right)$ application and in small sizes (10 kwatt), but these have not yet been optimized for high efficiency operation. Seippe] [13], of the Brown-Boveri Company, was the first to demonstrate an efficient gasdynamic energy exchanger, the "Cormprex," in which gas compression was accomplished by shock processes. The Comprex transferred 69 percent of the available work of expansion through a compression ratio of 2.5. Later, independent research was carried out by Kantrowitz et al. at Corne!1 University, Berchtold at ITE, and Zurich. At Cornell Aeronautics Laboratory (CAL, now CALSPAN), work continued on this type of machinery in the 1950s for proputsion, chemical processing, and a hypersonic wind tunnel. A prototype and a full-scale energy 


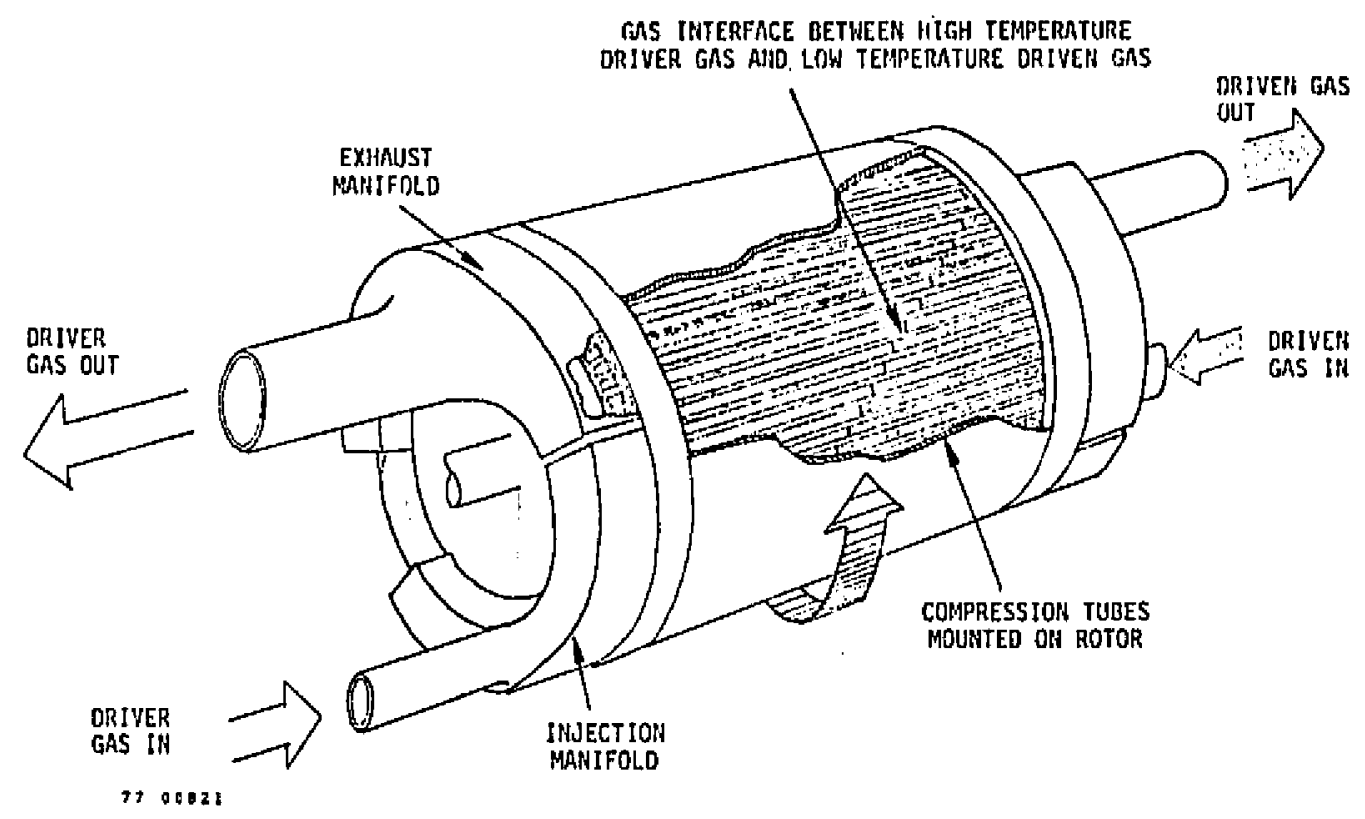

Figure 9. Cutaway View of Energy Exchanger with Rotating Tubes

exchanger device called a "wave superhea ter" were constructed at CAL and operated successfully for wind tunnel applications over a five-year period [14]. Temperatures as high as $4500^{\circ} \mathrm{K}$, with air flows of $5 \mathrm{lbs} / \mathrm{sec}$, were achieved in the full-scale wave superheater. It was not until 1966 that Weatherston and Hertzberg, utilizing their experience with the wave superheater, suggested the use of the energy exchanger as a means of circumventing the inlet temperature limitation of gas turbines [3].

The Cornell wave superheater was an important step towards the demonstration of high temperature, high mass throughput, energy exchanger operation. Figure 10 shows schematically how the multiple tube "gattling gun" design produced a quasi-steady stream of superheated air for flight simulation and re-entry studies. In this case, the compressed, heated air was withdrawn in a collector nozzle at its full temperature, effectively operating in reverse from an energy exchanger. Reversibility under ideal conditions is possible since all of the wave processes are isentropic; the $x-t$ wave diagram in Figure 8 can be reversed in time if the flow directions are also reversed.

The CAL wave superheater, utilizing air as a working substance (driven gas) and hel fum, preheated by pebble heaters, as the driver, produced a continuous jet of air at temperatures in excess of $4000^{\circ} \mathrm{K}$ with stagnation pressures of about $120 \mathrm{~atm}$. The only limit to the high temperature capability of this device proved 
to be the practical rotor tip speed which could be achieved. As a result of extensive preliminary design analysis and exploratory testing, the actual performance achieved agreed very closely with predictions, including maximum temperatures and pressures, interface mixing, and other losses. The machine was immediately put into use as an operating wind tunnel.

The straightforward structural design, utilizing broached tubes, proved to be exceptionally rugged, and few mechanical problems were encountered. Figure 11 shows a photograph of the wave superheater drum. The unqualified success of this device provides an important part of the foundation for renewed interest in wave machinery technology.

On a smaller scale (approximately $10 \mathrm{~kW}$ ), Brown-Boveri has started manufacturing a Comprex-Supercharger for Diesel engines,

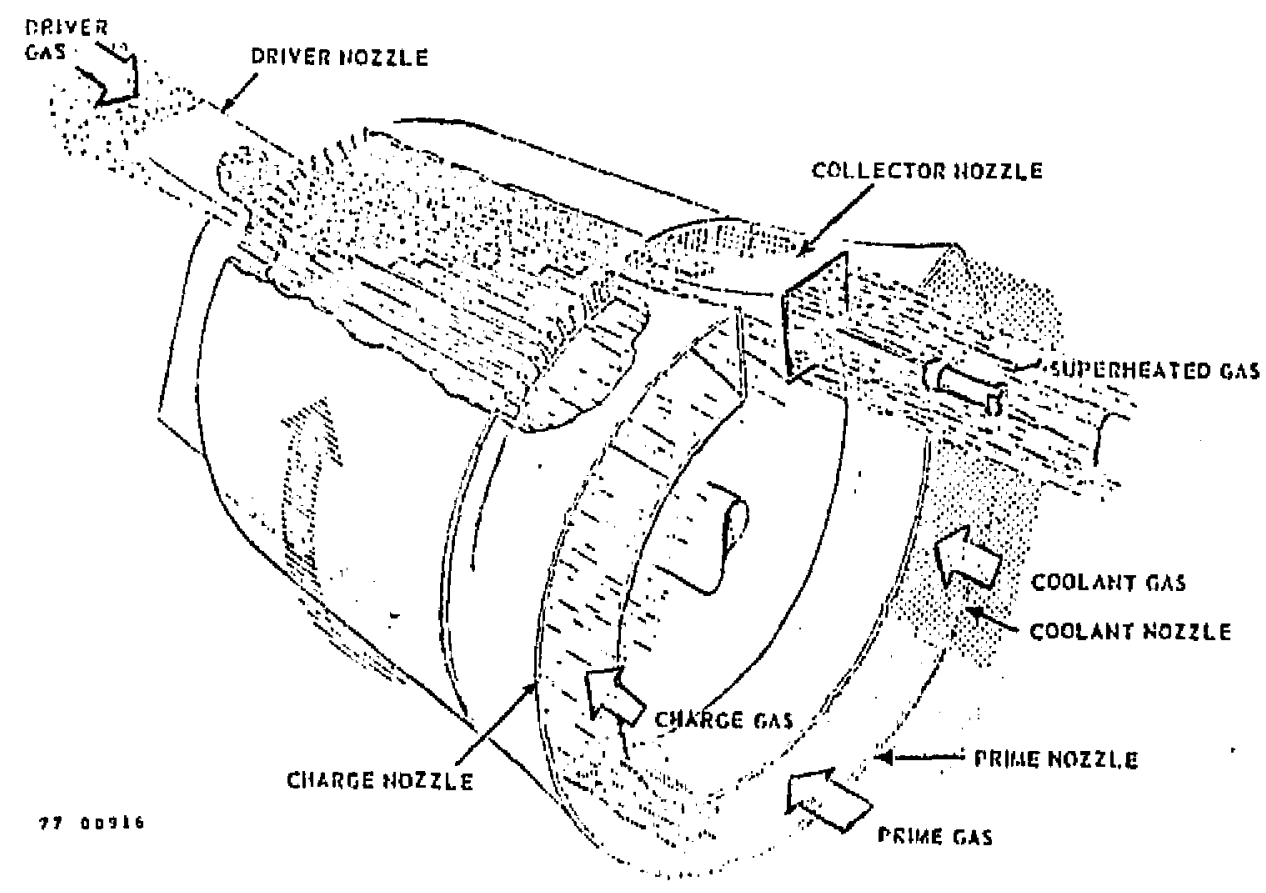

Figure 10. Isometric View of CAL Wave Superheater Used to Provide High Temperature Air for Re-entry Simulation 


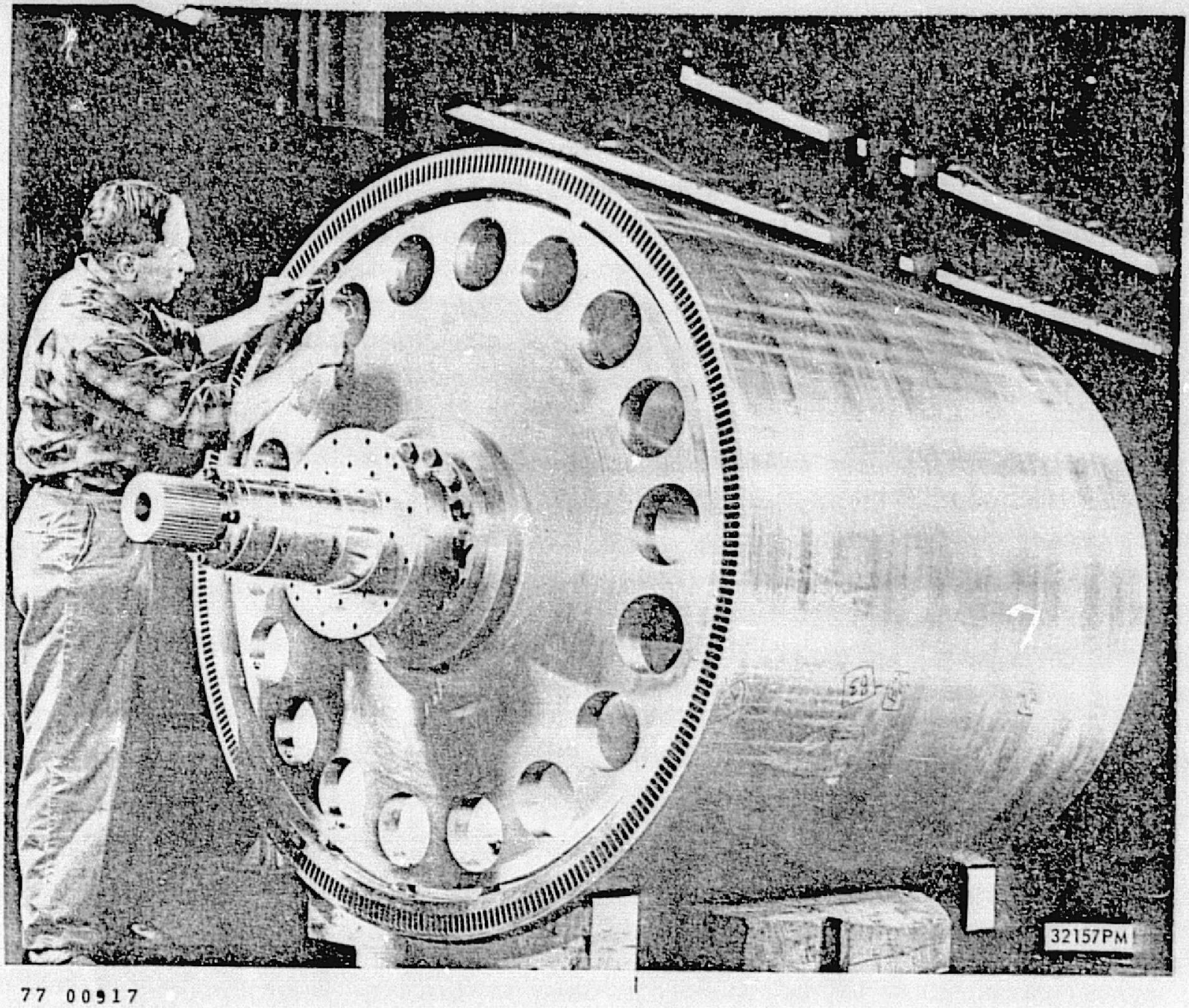

Figure 11. Photograph of the Full-Scale Gasdynamic Wave Heater Drum Constructed and Operated at CAL

shown schematically in Figure 12 [15]. Test data by BrownBoveri claim improvements in both power performance and economy over Seippel's original design, while also claiming beneficial effects on air pollution. In particular, the responsiveness of the Comprex-supercharger system is outstanding. This device is the first entry of a wave machine into the commercial market.

Additional work by Berchtold and his colleagues [16] on small energy exchanger applications to gas turbine automotive systems has demonstrated the increased thermal cycle efficiency which can be achieved by adding a Comprex-turbocharger to a rather low efficiency gas turbine (e.g., nTurb $\cong 20$ percent). No serious attempt was made in their studies to achieve high energy exchanger efficiency because the low turbine efficiency rapidly became the 


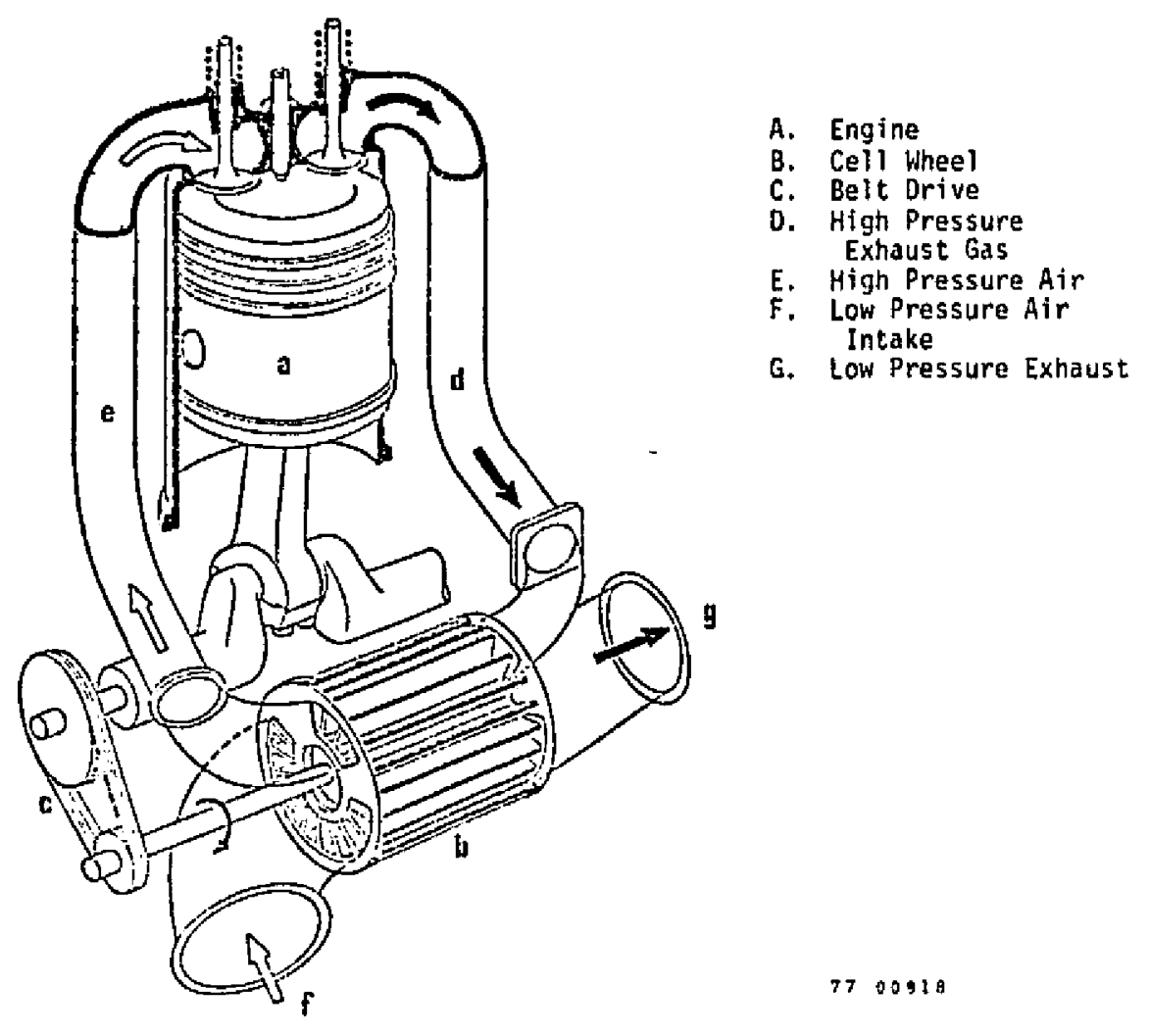

Figure 12. Schematic of the Supercharging Application of the Comprex Waveheater Currentiy Being Marketed by Brown-Boveri Company

limiting factor on total cycle efficiency. For the same reason, impedance matching was not an important consideration in their research. However, Berchtold et al. have addressed some of the practical design and engineering questions of the ducting and entrance/ exit nozzle manifolding required for all such machines.

The most recent study on energy exchanger technology, performed in 1977 at MSNW [17], explored the conceptual design, operation, material requirements, and peak cycle efficiency (on the order of 70 percent) for an energy exchanger used to boost the performance of an advanced fuels fusion reactor. The results of that study showed that the power conversion system employing an energy exchanger in a combined gas turbine/steam cycle would allow a relatively neutron-free fusion fuel to produce net electric power. No insurmountable technological barriers to developing that power conversion system were apparent. The potential for an efficient "hands-on" fusion reactor in facilitating long-term energy needs is clearly impressive. 
The principal loss mechanisms in the energy exchanger are leakage at the injection and exhaust nozzles, boundary layer losses in the tubes (e.g., heat transfer and viscous losses), mixing losses at the gas interface, and non isentropic shock compression [18]. Some of these losses are proportional to the inner surface area of the energy exchanger tubes; others are proportional simply to the circumference of the tubes or to the cross-sectional area of the tubes. A rather simple scaling of these losses with power throughput can be estimated by assuming that the only rotor parameters that change with power are the mass throughput, radius, length, and angular velocity. We assume that the tube length-to-tube diameter ratio stays fixed so that tube diameter also varies linearly with rotor length; the number of tubes also stays constant. Under these conditions,

$$
\begin{aligned}
& P \equiv \text { power } \propto r^{2} \quad(r=\text { rotor radius }) \\
& L=\text { rotor length } \propto r \\
& \dot{\theta}=\text { angular velocity } \propto r^{-1} \\
& \dot{m}=\text { mass flow } \propto r^{2} \\
& d=\text { tube diameter } \propto r
\end{aligned}
$$

With these relations, it can be shown that

$$
\begin{aligned}
& \text { leakage losses } \propto r \\
& \text { shock losses } \propto r^{2} \\
& \text { heat transfer losses } \propto r \\
& \text { viscous boundary layer losses } \propto r \\
& \text { mixing losses } \propto r^{2}
\end{aligned}
$$

Hence, those losses which scale as $r^{1}$ increase as $p^{\frac{1}{2}}$ with increasing power throughput; those losses which scale as $r^{2}$ increase as $P$. Only the former category of losses, therefore, permit an increase in efficiency with larger power devices. However, the shock and mixing losses can be reduced to much lower levels in larger devices by redesigning the rotor so that a greater number of compression waves are used, and so that the gas purification procedures demand a sinaller fraction of recirculated power from the system. Since 
the performance of a small energy exchanger will be diminished mostly by leakage and boundary layer losses, the scaling derived above is reasonably optimistic, assuring much better performance as the size and power output of the device increases.

The gas turbine is subject to somewhat unique conditions for high temperature cycles, since the working fluid entering the turbine from the energy exchanger can be at quite a low temperature. Consequently, the thermal sealing losses in relatively small gas turbines can be minimized because of low temperature operation (i.e., machined tolerances will be closer to actual tolerances during operation). Other problems will arise, however, since lighter molecular weight fluids, such as helium or helium/argon mixtures (monatomic gases), must be used as the driven gas in the energy exchanger to reach these low turbine inlet temperatures. That is, the impedance matching condition of the energy exchanger, which states that

$$
T_{\text {inlet }} \equiv T_{\text {driven }}=\frac{m_{\text {driven }}}{m_{\text {driver }}} \quad T_{\text {driver }}
$$

requires that the molecular weights satisfy $m_{d r i v e n}<m_{d r i y e r}$. For peak driver gas temperatures of $3000^{\circ} \mathrm{K}$ and turbine inlet temperatures limited to $1167^{\circ} \mathrm{K}$ (i.e., $2100^{\circ} \mathrm{R}$ ), the desired molecular wejght ratio is miven $/$ m driver $\cong 0.40$.

Low temperature, low molecular weight working fluids can approach Mach number limits in multi-stage turbines, and so shock losses will be important. In small systems, radial inflow turbines, with reduced sealing and shock losses, are the most efficient form of turbine to use [19]. Such turbines have been developed for cars, airplane air conditioning systems, hel icopters, and power plants. A 3-kW radial inflow turbine for SNAP 8 was developed by Garrett AiResearch [20], and there is a we11-documented history of space technology at the size level intended for the demonstration experiment presented in Section IV.

A variety of gas purification procedures exists for the energy exchanger/turbine combination. These are:

1) Distiliation

2) Chemical separation

3) Centrifuging

4) Membrane diffusion

5) Aerodynamic separation (e.g., turning flow) 
Membrane or porous plug diffusion typically requires large pumping power (i.e., low separative power rer stage), but has the advantage of large throughputs. Distillation requires either driver or driven working fluids be condensible. Only partial tiquefaction may be needed to achieve sufficient separation, if only a small quantity of impurity is introduced per unit time. Centrifuging and aerodynamic separation both utilize centrifugal forces, the latter requiring no moving parts in a high speed turning flow. Aerodynamic separation has heen included in previous designs for spacecraft power plants which utilized a liquid metal MHD system [21].

Natural centrifuging already occurs in the turbine itself. It is sufficient to bleed off part of the peripheral flow in a turbine at $60,000 \mathrm{rpm}$ to extract a portion of the heavier molecular weight components. For example, a mix of xenon and helium could be used both as the driver and as the driven working fluids; by varying the percentages of each of these gases, impedance matched conditions would give appropriate turbine inlet temperatures. With a mass difference of $131-4=127$, centrifugal separation in the driven gas loop would be very effective.

From a consideration of ideal regenerated and intercooled cycles, it was found that the best configuration for this engine consists of three intercooled compressors, and one or possibly two turbines fed by an equivalent number of energy exchangers, as shown schematicaliy in Figure 13. The expression for the actual cycle

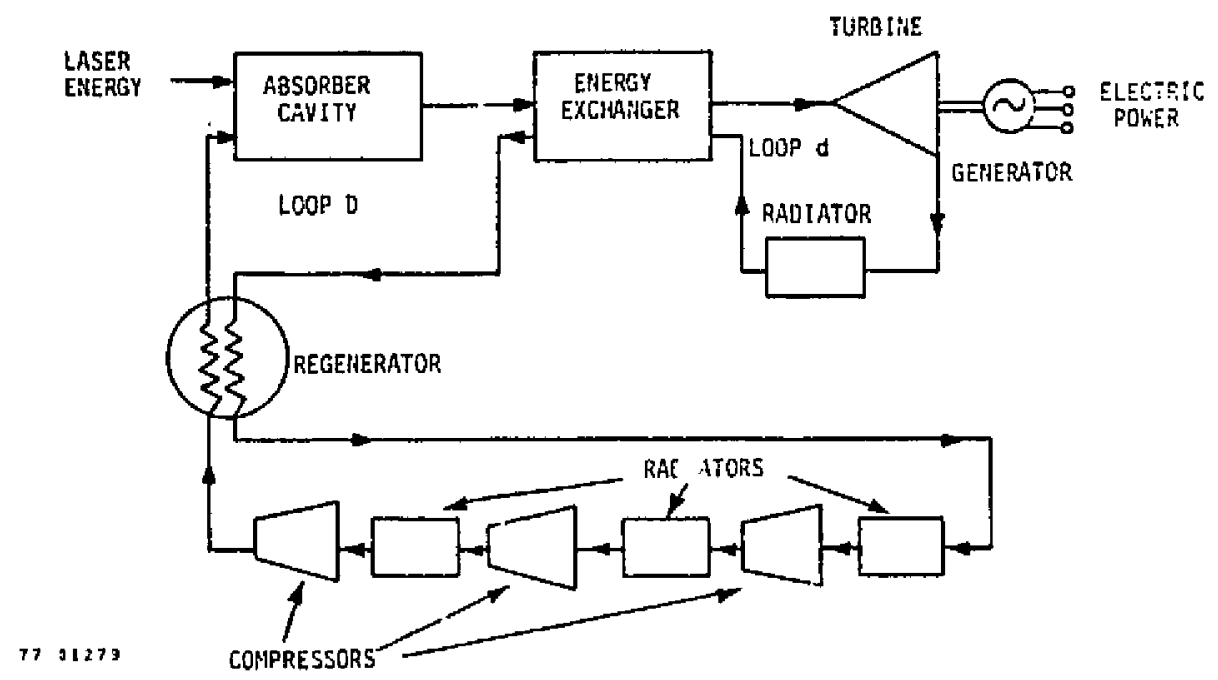

Figure 13. Schematic of Intercooled, Regenerated Brayton Cycle Utilizing an Energy Exchanger for High Efficiency Operation 
efficiency is

$$
\left.n=\frac{k^{-1}\left(1-\tau_{t d}\right)-3 \tau^{-1}\left(\tau_{c D}-1\right)}{1-\left[\varepsilon \tau_{t D}{ }^{+}(1-\varepsilon) \tau^{-1}{ }^{\tau} c D\right.}\right]
$$

where

$$
K=\left\{n_{t d}\left[1-\left(1-n_{c d}\right) \pi_{c d}^{-\frac{\gamma-1}{\gamma}}\right]\right\}^{-3}
$$

represents the effects of the energy exchanger component efficiency (which is equal to $\left(n_{t} n_{c d}\right)^{1 / 2}$ ), $n_{t}$ and $n_{c}$ are the turbine and compressor efticiencies for the $D$ (driver) and $d$ (driven) loops shown in Figure 13 and $\varepsilon$ is the regenerator effectiveness. The values of $n_{t D}$ and $n_{c d}$ are taken to be equal, and each is equal to the square root of the energy exchanger efficiency. The computation of the temperature ratios, $\tau_{D}$ and $\tau_{d}$, and pressure ratio, $\pi_{d}$, can be obtained by prescribing the peak-to-minimum cycle temperature ratio, $\tau$, and the pressure ratio, $\pi_{C D}$, for a single compressor. The equations relating these quantities are:

$$
\begin{aligned}
& \pi_{\mathrm{Cd}}=\pi_{0}\left(\pi_{\mathrm{CD}}\right)^{3} \\
& \tau_{\mathrm{td}}=1-n_{\mathrm{td}}\left[1-\left(\pi_{r} \pi_{\mathrm{cd}}\right)^{-\frac{\gamma-1}{\gamma}}\right] \\
& \tau_{\mathrm{CD}}=1+\frac{1}{n_{\mathrm{CD}}}\left(\pi_{\mathrm{cD}}^{\frac{\gamma-1}{\gamma}}-1\right) \\
& \pi_{\mathrm{tD}}=\pi_{\mathrm{cd}}^{-1} \\
& \tau_{\mathrm{tD}}=1-n_{\mathrm{tD}}\left(1-\frac{\gamma-1}{\gamma}\right)
\end{aligned}
$$

Using component efficiencies for actual turbines and compressors generating at a 3-kwatt power level, estimated energy exchanger efficiencies and pressure drops in radiators, heat exchangers, 
etc. of $0.97=\pi_{r}=\pi_{0}^{1 / 6}$ per component, we find a net overa 11 cycle efficiency of 56 percent for $\tau=10, \eta_{E E}=0.85$. Note from Figure 14 that the maximum efficiency for $\tau=F \delta$ could exceed 56 percent, but that the regenerator temperature $T_{5}$ becomes too high at that point (i.e., $1170^{\circ} \mathrm{K} \cong 2100^{\circ} \mathrm{R}$ is taken as an upper limit on current regenerator material technology). Lower energy exchanger component efficiencies (i.e., 70 percent), also shown in Figure 14 , do not lower the peak cycle efficiencies proportionately but do result in unacceptably high regenerator temperatures. These values suggest that with even the best component efficiencies, an adequate cycle efficiency can only be achieved with a CO laser (i.e., for $T_{\max }=$ $3000^{\circ} \mathrm{K}, \mathrm{T}_{\min }=300^{\circ} \mathrm{K} ; \tau=10$ ). Furthermore, energy exchanger efficiencies of 85 percent at the 1-kwatt level still need to be demonstrated, and are not available as near-term technology. In Section 3.7, it is shown that as the engine size increases, higher cycle efficiencies can be obtainad, even with energy exchanger component, efficiencies less than 85 percent, possibly as low as present Comprex efficiencies (i.e., about 70 percent).
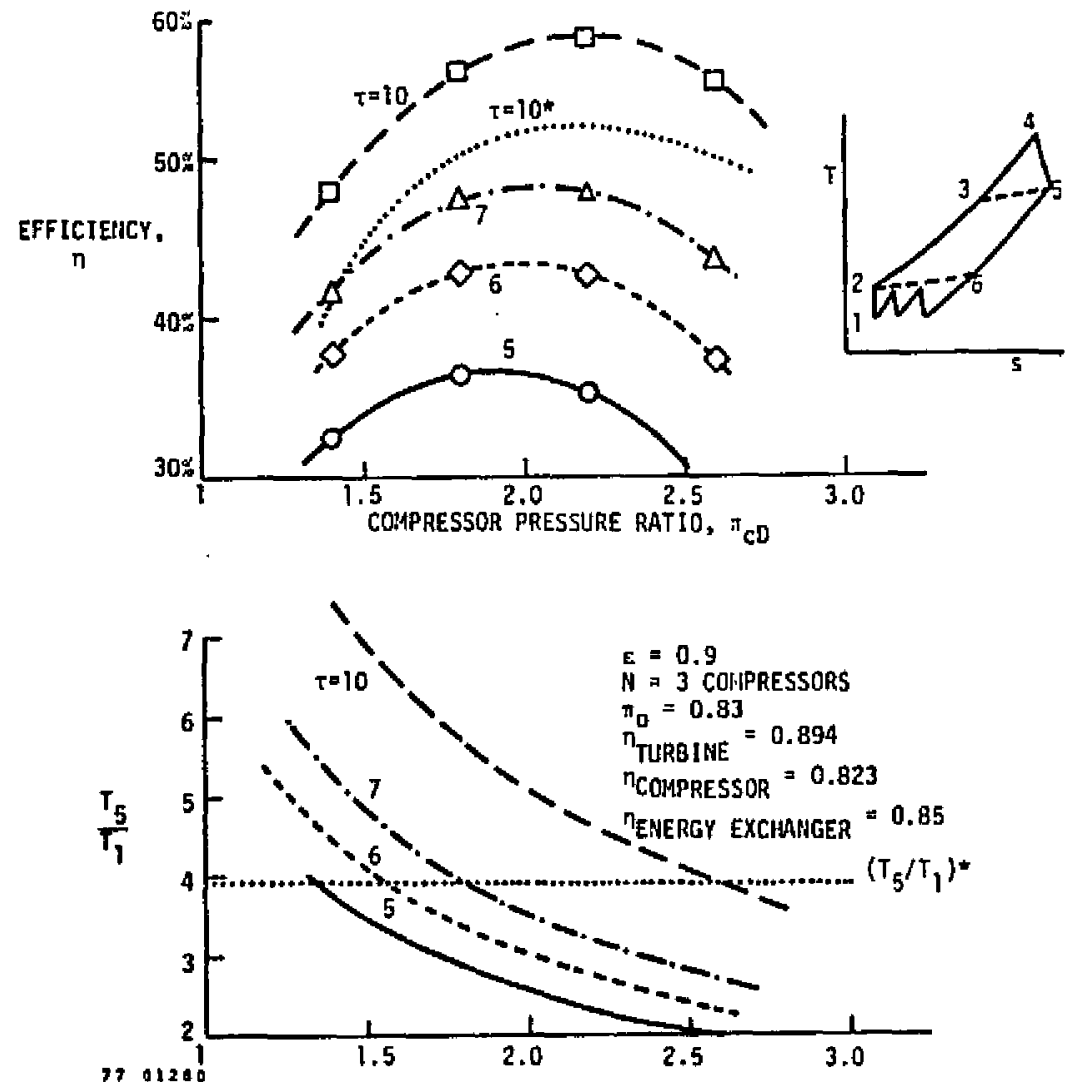

Figure 14. Energy Exchanger/Turbine Efficiency and Regenerator Peak Temperature $\left(T_{5} / T_{1}\right)$ as Functions of Maximum-toMinimum Temperature Ratio $\tau \equiv T_{4} / T_{1}$ and Compressor Ratio $\pi C D\left[\tau=10^{*}\right.$ for $n_{E E}=70 \%$ instead of $80 \%$; $\left(T_{5} / T_{1}\right){ }^{\mathrm{CD}}$ corresponds to $T_{5}=1170^{\circ} \mathrm{K}$ when $\left.T_{3}=300^{\circ} \mathrm{K}\right]$ 


\subsubsection{Piston Engines}

A variety of piston engines have been considered for space applications, including the Stirling cycle engine $[7,9]$ and the 0tto cycle engine $[7,22]$. These can be classified as internaliy or externally heated engines. Typically, internally heated engines have been associated only with chemical fuels and have therefore been restricted to short space missions. Longer missions have utilized externally heated engines supplied by solar or nuclear power. A prime example of an externally heated piston engine is the continuously heated Stirling engine. Unfortunately, externally heated engines are limited to low temperatures compatible with the temperature constraints imposed by operating the piston with a hot cylinder wall. This places a low Carnot limit on the engine thermal efficiency.

Laser energy has the special attribute that it can be delivered in pulses through a transparent window and absorbed directly in the engine working fluid, allowing an engine to be internaliy heated. Because the walls of an internally heated engine can be kept cool while the working fluid reaches very high temperatures, the thermal efficiency of such engines can be theoretically much higher than the externally heated engine.

By adjusting the laser heating pulse, either constant volume or constant pressure heat addition can be achieved, making it possible to use either 0tto or Diesel cvcles, respectively. 0ther thermodynamic cycles, such as the resonantly operated Stirling cycie or the hybrid cycle developed by Carbuny and Pechersky [5], can also be used by adapting the laser pulse characteristics. As pointed out by the latter authors, additional increases in efficiency are possible with laser driven engines because they can utilize monatomic instead of polyatomic working fluids. For example, the otto cycle efficiency is given by

$$
\eta_{\text {th }}=1-\frac{1}{r^{\gamma-1}}
$$

( $r$ is the compression ratio and $\gamma$ is the ratio of specific heats). For a given compression ratio, the efficiency will increase when a monatomic gas is used.

A schematic of a laser driven piston engine is shown in Figure 15. The basic components specific to this engine are: 


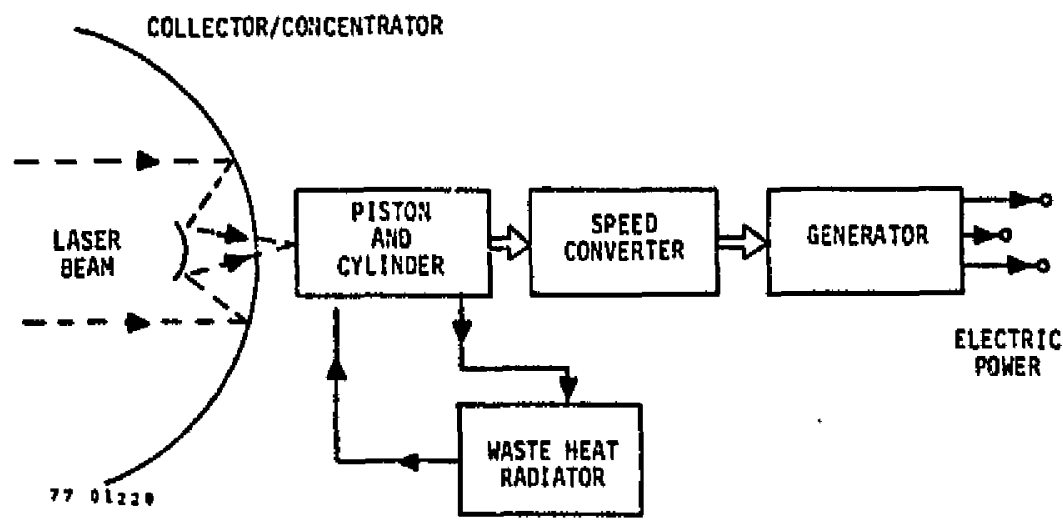

Figure 15. Schematic for a Piston Laser Heat Engine

- Piston

- Cylinder

- Orive shaft and flywheel (or linear extension)

- Speed converter (e.g., gear box)

- Valves and exhaust and entrance manifolds

The upper temperature limit on pistons and cylinders appears to be about $585^{\circ} \mathrm{K}$ when lubrication is required; unlubricated walls (e.g., for the externally heated Stirling cycle) can reach temperatures of $1120^{\circ} \mathrm{K}$ with current materials technology. Advances using refractory metals and ceramics may allow these temperatures to approach $1600^{\circ} \mathrm{K}$. However, even the most ambitious ground-based development program today is aiming at much more modest temperatures for the 1985 time period. Peak pressures in high compression piston engines can be as high as 1500 psi (i.e., Diesel engines).

Because this engine nust survive for long periods without maintenance, lubrication must be economical. For this reason, an engine cycle seems desirable where the crankcase oils are segregated from the working fluid as much as possible, or where dry lubrication is used. clearly, some contamination of the working fluid and upper piston chamber by liquid or solid lubricants will occur which may interfere with the energy absorption process by ccating the inner surface of the window and by chemically interrupting the abscrption of laser radiation by the "seed" molecules. The latter effect must be evaluated for each particular mix of seed and lubricants used. Gas cleanup is required so that the concentration of polyatomic molecules in the working fluid does 
not change the thermodynamic properties (e.g., $\gamma=5 / 3$ ) appreciably, and does not contribute too much to radiative losses.

The problem of window fouling can be approached by bringing part of the cold intake gas across the window face. This will help to wipe it clean and to cool the window. Also, the laser radiation may be absorbed preferentially by the lubricant, vaporizing it from the inner window surface. Careful design may heip to mitigate this problem.

Piston Engine Scaling. Rather general principles of similitude have been developed for internal cornbustion engines to scale engines with comparable design to Jarger sizes [23]. The basic assumption is that the characteristic dimension of each part of an engine scales upwards in size so as to preserve the ratios of size between each part of the engine. Then, the stroke-to-bore ratios are equal, the compression ratios are equal, the volumetric efficiencies (and mean flow velocities) are equal for equal mean piston speeds, and the specific gas pressures are equal. Under these conditions,

$$
\begin{aligned}
& \text { Rpm } \propto d^{-1} \\
& \text { Displacement } \propto d^{3} \\
& \text { Woight } \propto d^{3} \\
& \text { Bearing areas } \propto d^{2}
\end{aligned}
$$

where $d$ is any characteristic dimension, such as the cylinder bore. Thus, power which depends on compression ratio (i.e., mean effective pressure) and displacement per unit time, is proportional to $d^{-1} \cdot d^{3}=$ $d^{2}$.

The specific power of similar engines therefore scales as

$$
\text { Power/Weight } \propto d^{2} / d^{3} \propto d^{-2}
$$

implying an advantage for small cylinder engines. The ideal efficiency of similar engines is fixed by the compression ratio for the otto cycle, by both the compression ratio and the constant pressure expansion ratio for the Diesel cycle, and by peak and minimum temperatures for the Stirling cycle. Hence, the ideal thermal efficiency for each of these cycles is constant for similar engines.

$$
\begin{aligned}
& \text { ORIGINAL PAGE IS } \\
& \text { OF POOR QUALITY }
\end{aligned}
$$


Frictional losses occur between che piston and cylinder walls and in all of the bearings. The piscon losses depend on oil viscosity, the shearing area, oil-film thickness, and the piston speed. To achieve the same bearing aperating conditions in similar engines requires that the product of $\mu \dot{\theta} / p$ be constant $(\dot{\theta}=$ engine rpm). Since the specific bearing load, $p$, is constant (for equal piston speeds), this implies that $\mu \dot{\theta}=$ const, or that $\mu \sim \mathrm{d}^{1}$ for the same bearing temperature. Thus, we may assume a different lubricant for larger engines to accomodate the shift in $\mathrm{rpm}$ with size. If we are constrained to the same lubricant because of other conditions, such as material compatibility or mission reliability, then a different class of friction loss scaling will obtain. Both are examined next.

First, for $\mu \sim d^{l}$, we have the piston friction loss proportional to $\mu \cdot A \cdot \delta^{-1} \cdot v_{p}\left(A=\right.$ shearing area $\propto d^{2}, \delta \propto d, v_{p}=$ const piston speed), or tb $\mathrm{dd}^{2} \mathrm{~d}^{-1}=\mathrm{d}^{2}$; for a fixed lubricant, the piston friction is proportional to $d^{2} d^{-1}=d$, but the bearing operating conditions will become more severe with increasing size. The heat losses are

$$
Q=\text { rate } \cdot \text { area } \cdot \Delta T
$$

The: temperature conditions should be the same for similar engines so that the heat transfer rate coefficients are the same, and therefore,

$$
\oint_{\text {cycle }} \alpha \text { const } \cdot d^{2}
$$

Thus, both heat and frictional losses are proportional to $\mathrm{d}^{2}$. The ideal horsepower is proportional to the compression ratio and the displacement per minute, and hence, depends on const $\cdot d^{3} \cdot d^{-1} \propto$ $d^{2}$. Thus, the mechanical efficiencies are independent of $d$ for $\mu \propto d^{d}$. If $\mu$ is independent of $d$, then the mechanical efficiency will increase with $d$.

Heat transfer losses are the dominant effect in decreasing the efficiency of piston engine operation. Heat conduction calculations for laser heated piston engines were presented by Martini [7] for both Otto and GP cycles showing a qualitatively correct reduction in available work. We have extended Martini's calculations to include heat transfer through a convective boundary layer. For both helium and argon working fluids, the Reynolds number at relatively low piston $\mathrm{rpm}$ (e.g., 100 to $500 \mathrm{rpm}$ ) is still in the 
laminar region so that a laminar boundary layer heat transfer calculation is appropriate. Macroscopic convection in the cyl inder due to gas intake and exhaust, particularly for two-cycle engines, will probably be strong enough to thermally mix the gases shortly after laser heating so that a nearly uniform temperature profile is achieved in a short time compared to the downstroke. Hence, we have assumed a uniform gas temperature which is changed by expansion or compression and by heat transfer through the boundary layer.

In order to obtain an accurate measure of an 0tto cycle operating with a monatomic gas, that is, similar in every other respect to the combustion case, we have chosen to scale experimental data available for a combustion otto cycle to conditions appropriate to a monatomic gas. For a $6.2: 1$ compression ratio, the heat loss through the cylinder, the availability of that heat to do work, and the net loss in available work (product of column 1 and column 2) are shown in Table 4 [24].

Table 4

Distribution and Avajlability of Heat Loss

$(\gamma=1.27, \quad C R=6.2)$

\begin{tabular}{lccc}
\hline PROCESS & $\begin{array}{c}\text { HEAT LOSS } \\
(\%)^{*}\end{array}$ & AVAILABILITY & $\begin{array}{c}\text { LOSS DF } \\
\text { AVAILABLE WORK (\%) }\end{array}$ \\
\hline Compression & 0.5 & 0.143 & 0.07 \\
Combustion & 7.0 & 0.392 & 2.74 \\
Expansion & 9.0 & 0.143 & 1.29 \\
Exhaust** & 17.0 & 0.0 & 0.0 \\
& 33.5 & & 4.1 \\
& TOTAL & Expressed as a percent of total heat input for the ideal cycle. \\
** Not the heat in the exhaust gases; that is 27.3 percent.
\end{tabular}

In actual operation, work is being done against friction. This work turns up as heat both in the cylinder wall and in the combustion gases. In addition, heat is lost from the combustion gas before it can be used to do work, and energy is lost through pumping work. For the present example, with $\gamma=1.273$, the energy is distributed as follows: 


$$
\begin{gathered}
\left(W_{\text {out }}\right)_{\text {ideal }}=n Q_{\text {in }}=\left(1-\frac{1}{r r-1}\right) Q_{\text {in }}=0.392 Q_{\text {in }} \\
W_{\text {pump }}=0.016 Q_{\text {in }} \\
Q_{\text {out }}=0.608 Q_{\text {in }} \\
W_{\text {frict }}=0.08 Q_{\text {in }} \\
\left(W_{\text {out }}\right)_{\text {actual }}=0.296 Q_{\text {in }}
\end{gathered}
$$

We shall assume that the monatomic otto cycle has the same compression ratio, uses the same piston, cylinder, and crank-topiston assembly (that is, the displacement, $\chi$, has the same functional relationship to the crank angle, $\theta$ ), operates at the same minimum cycle pressure, utilizes the same minimum and maximum cycle temperatures, and delivers the same power as the combustion 0tto cycle. The monatomic cycle therefore has the following properties (see Fig. 16 comparing both cycles),

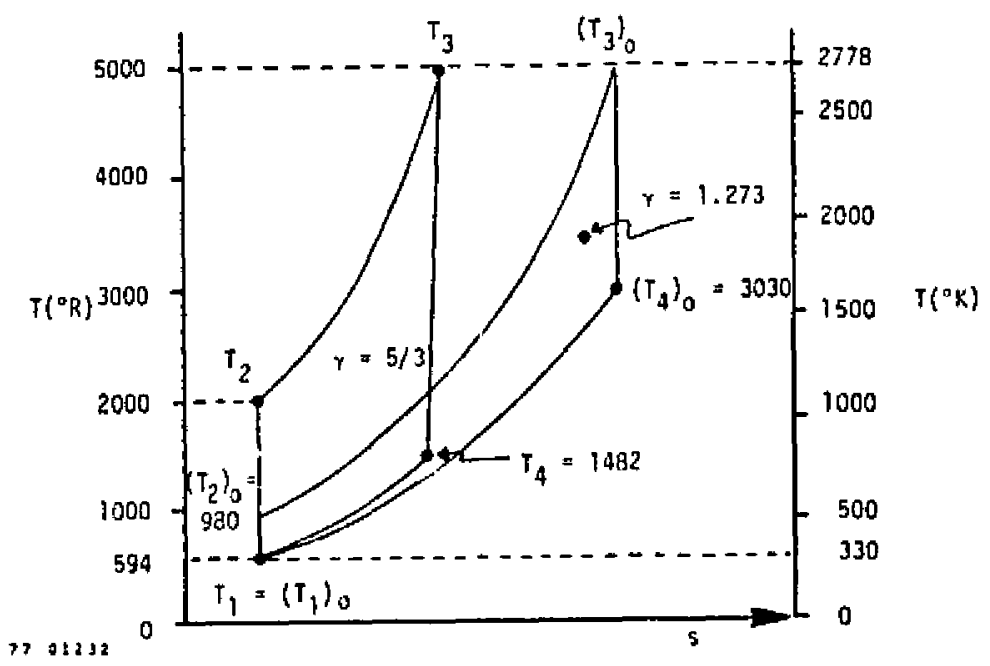

Figure 16. Comparison of Combustion $(\gamma=1.273)$ and Monatomic Gas $(\gamma=5 / 3)$ Otto Cycles 


$$
\begin{gathered}
\eta_{\text {ideal }}=1-\frac{1}{(6.2)^{0.067}}=0.704 \\
T_{2}=3.37 \mathrm{~T}_{1} \\
T_{4}=0.296 \mathrm{~T}_{3} \\
Q_{\text {exh }}=\frac{c_{p} T_{4}}{\left(c_{p} T_{4}\right)_{0}} Q_{\text {exh }(0)}=\frac{\gamma-1}{\gamma}\left(\frac{\gamma-I}{\gamma}\right)_{0} \frac{T_{4}}{T_{0}}\left(Q_{\text {exh }}\right)_{0}=0.072 Q_{\text {in }} \\
W_{\text {pump }}=\left(\frac{\gamma_{0} R_{0} T_{0}}{\gamma R T_{1}}\right)^{1 / 2}\left(W_{\text {pump }}\right)_{0}=0.014 Q_{\text {in }}
\end{gathered}
$$

where the subscript " 0 " refers to the combustion 0tto cycle. To maintain the power outputs the same, with possible differences in the energy per cycle, requires a change in the engine rpm in these two cases. Specificalty, $P \equiv$ power $\propto W_{c} \dot{\theta}$, where $\theta$ is the rpm and $W_{c}$ is the work per cycle. Therefore,

$$
\dot{\theta}=\frac{\left(W_{c} \dot{\theta}\right)_{0}}{W_{c}}=1.818 \dot{\theta}_{0}
$$

The frictional work is proportional to the rpm (or piston speed), so that

$$
W_{\text {frict }}=1.818\left(W_{\text {frict }}\right)_{0}=0.15 Q_{\text {in }}
$$

The heat transfer losses can be estimated for each part of the cycle shown in Table 5 by prorating the losses according to the mean temperature in the appropriate part of the stroke (see Fig. 16). 
Table 5

Distribution of Availability Losses

$$
(\gamma=5 / 3, C R=6.2)
$$

\begin{tabular}{|c|c|c|c|}
\hline PROCESS & HEAT LOSS $(\%)$ & AVAILABILITY & WORK LOSS $(\%)$ \\
\hline Compression & $\frac{1297}{787}(0.5)=0.8$ & 0.20 & 0.16 \\
\hline $\begin{array}{l}\text { Combustion } \\
\text { (Heat Addition) }\end{array}$ & $\frac{3500}{2900}(7)=8.2$ & 0.70 & 5.74 \\
\hline Expansion & $\frac{3240}{4015}(9)=7.3$ & 0.20 & 1.46 \\
\hline Exhaust & $\frac{1038}{1812}(17)=9.7$ & 0.0 & 0.0 \\
\hline TOTAL. & 26.0 & & 7.36 \\
\hline
\end{tabular}

where the availability is given by the formula

$$
A=n\left\{\frac{\left(\frac{x_{\max }}{x}\right)^{\gamma-1}-1}{\left(\frac{x_{\max }}{x_{\min }}\right)^{\gamma-1}-1}\right\}
$$

where $x$ is the piston position. Clearly, A depends on $\gamma$ and will be larger at top dead center and fall off more precipitously as the piston is extended. The availability for the compression and expansion strokes is shown in Table 5, and is taken as the simple integrated mean of the availability over the entire piston extension. Thus, $Q_{\text {exh }}+Q_{\text {heat trans }}=0.332 Q_{\text {in }}$.

Real Cycle Analysis. It is now possible to estimate the effect of heat transfer, friction, and pumping work losses on the ideal efficiency for the monatomic gas cycle, as follows. The actual work performed will be 


$$
W_{\text {act }}=W_{\text {ideal }}-W_{\text {ht.red }}-W_{\text {frict }}-W_{\text {pump }}
$$

where $W_{\text {ht. red }}=0.074 Q_{\text {in }}$ is the reduction in available work due to heat transfer, and $W_{\text {frict }}=0.15 Q_{\text {in }}$, and $W_{\text {pump }}=0.014 Q_{\text {in }}$, to give

$$
W_{\text {act }}=W_{\text {idea }}-0.238 Q_{\text {in }}
$$

Also, the actual heat input will differ from the ideal heat input because the cycle diagram has been altered by the loss processes. Thus,

$$
\left(Q_{\text {in }}\right)_{\text {act }}=\left(Q_{\text {out }}\right)_{\text {act }}+W_{\text {act }} \text { (Conservation of Energy) }
$$

Since

$$
\left(Q_{\text {out }}\right)_{\text {act }} \cong\left(Q_{\text {out }}\right)_{\text {idea }}+Q_{\text {ht.trans }}
$$

and

$$
\left(Q_{\text {out }}\right)_{\text {ideal }}=\left(Q_{\text {in }}\right)_{\text {idea } 1}-W_{\text {idea } 1}
$$

We have

$$
\begin{aligned}
\left(Q_{\text {in }}\right)_{\text {act }} & =\left(Q_{\text {out }}\right)_{\text {idea }}+Q_{\text {ht.trans }}+w_{\text {ideal }}-W_{\text {ht.red }}-W_{\text {frict }}-W_{\text {pump }} \\
& =\left(Q_{\text {in }}\right)_{\text {ideal }}+Q_{\text {ht.trans }}-W_{\text {ht.red }}-W_{\text {frict }}-W_{\text {pump }} \\
& =\left(Q_{\text {in }}\right)_{\text {ideal }}(1+0.26-0.074-0.15-0.014) \\
& =1.02\left(Q_{\text {in }}\right)_{\text {ideal }}
\end{aligned}
$$

and

$$
n_{\text {act }}=\frac{W_{a c t}}{\left(Q_{\text {in }}\right)_{a c t}}=0.45
$$


By comparison, for the combustion Otto cycle, $\eta_{\text {ct }}=0.21$. Thus, the use of $\gamma=5 / 3$ rather than $\gamma=1.273$ has molet than doubled the actual thermal efficiency of the Otto cycle for the same power output. The reduction from 70.3 percent to 45 percent due to real cycle processes amounts to a $70.3-45 / 70.3=36$ percent decrease in ideal efficiency. It is worth noting that this monatomic 0tto cycle is nearly the maximum work cycle for the maximum and minimum temperatures prescribed. The heat transfer results obtained by scaling here have been corroborated by more detailed calculations using the convective boundary layer method described earlier for helium; smaller heat losses will occur if argon is used instead, as shown in Figure 17, where only heat losses are accounted for.
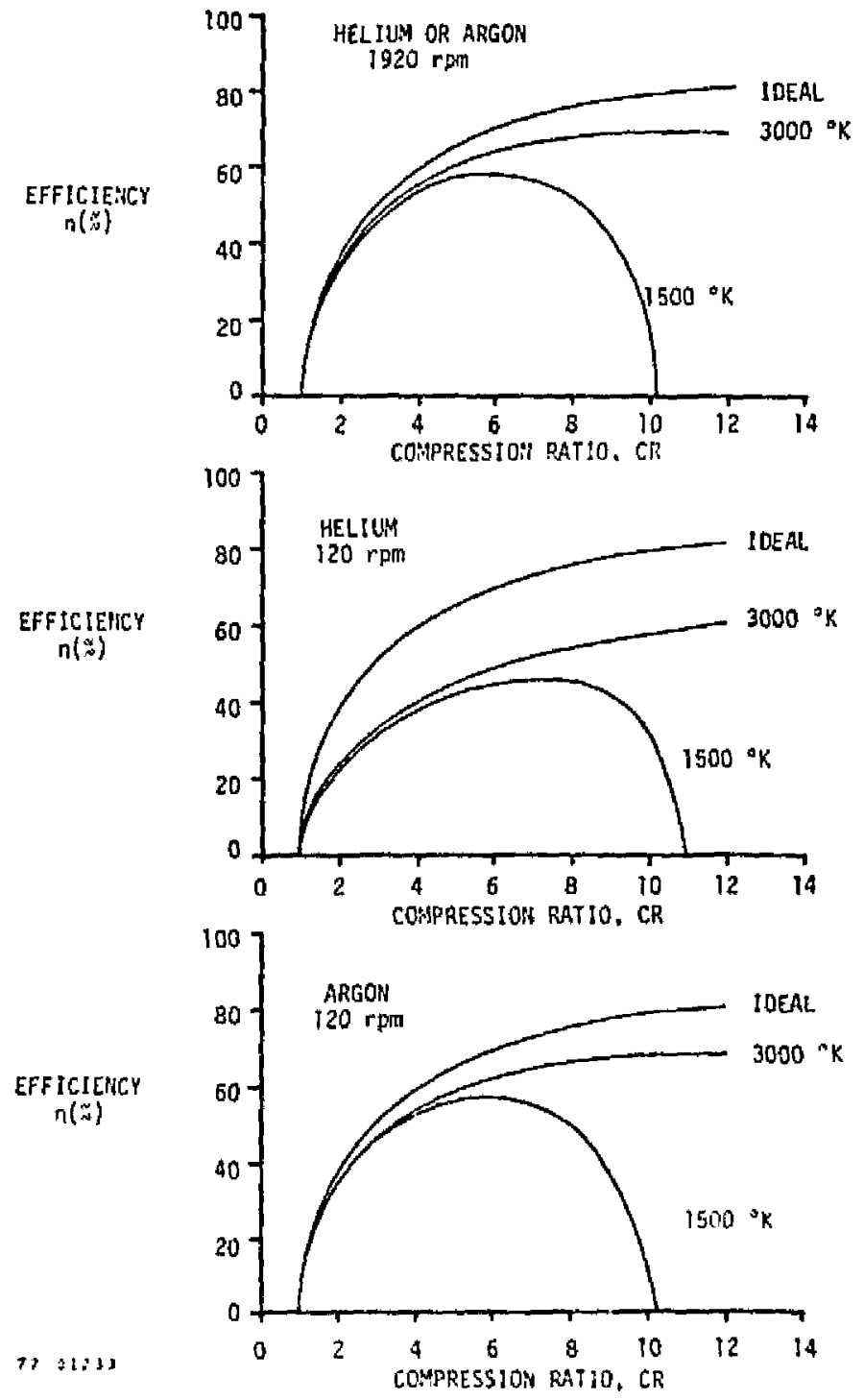

Figure 17. Effects of Heat Transfer on Otto Cycle Efficiency for Different Peak Temperatures and Working Fluids 
If we consider a comparable Diesel cycle, operating between the same maximum and minimum temperatures and yielding the maximum work for the same minimum cycle pressure and maximum volume, a compression ratio of approximately 12 is required, and the ideal efficiency rises to 76 percent.

Suppose that a limit of 1500 psi is placed on the cylinder peak pressure, commensurate with current technology. Starting with a minimum pressure of $14.7 \mathrm{psi}$, a total pressure rise of 102 occurs in the engine. For an otto cycle, the adiabatic compression achieves a factor of 64 increase in the initial pressure, and an additional factor of 1.6 increase in pressure occurs during the constant volume heating. Thus, a compression ratio of $(64)^{1 / \gamma} \approx 12$ is required, and the ideal otto cycle efficiency is 81 percent. The actual efficiency for this cycle can be scaled from the results of the 6.2 compression ratio 0tto cycle engine for $\gamma=5 / 3$, by assuming that the maximum volume, minimum cycle pressure, and rpm are the same, but that the displacement volume is increased because the minimum volume in the cycle is decreased. Under these conditions, the work output per cycle is reduced (i.e., this is no longer the maximum work cycle), so that the power output is also reduced. The results of this calculation are summariced in Table 6 . The actual efficiency is 55 percent, to within \pm 5 percent. Tin is is quite an acceptable engine cycle, judging from she actual thermal efficiency, pressure, and temperatures resulting from our calculations.

A survey of Stirling technology was performed to evaluate the potential for incorporating either resonant or black-body absorption of laser radiation into the engine cycle. Major efforts are currently underway to develop Stirling engines for automotive, remote power, and agricultural uses. Engine sizes vary from multicylinder, 200-kW units to small, free-piston versions in the 1-kW range.

The Phillips Company of Eindoven, The Netherlands, has been the leader in Stirling engine development. An American subsidiary of Phillips, in conjunction with the Ford Motor Company, is working on development of high efficiency, automotive power plants for future application. A parallef program by the team of United Stirling of Sweden and Mechanical Technology, Inc. (MTI) is also underway. Both efforts are funded by the Department of Energy and are being supervised by NASA-Lewis research center. General Electric is the systems contractor with American Phillips, the principle subcontractor for a l-kW radioisotope heated Phillips Stirling engine driven remote power unit. The target efficiency for this unit is $3 \mathrm{C}$ percent (heat to electricity) by 1979. MTI is also developing a 1-kW remote power unit. This system involves a linear Beale free piston engine driving a linear alternator. An overall conversion efficiency of 35 percent by 1985 is the goal of this program. JPL is currently investigating a free piston Stirling engine with a window in the hot space. The application will utilize solar energy to pump water for agricultural uses. 
Table 6

Comparison of $r=12$ to $r=6.2$ for $a$

Monatomic Gas Otto Cycle Engine

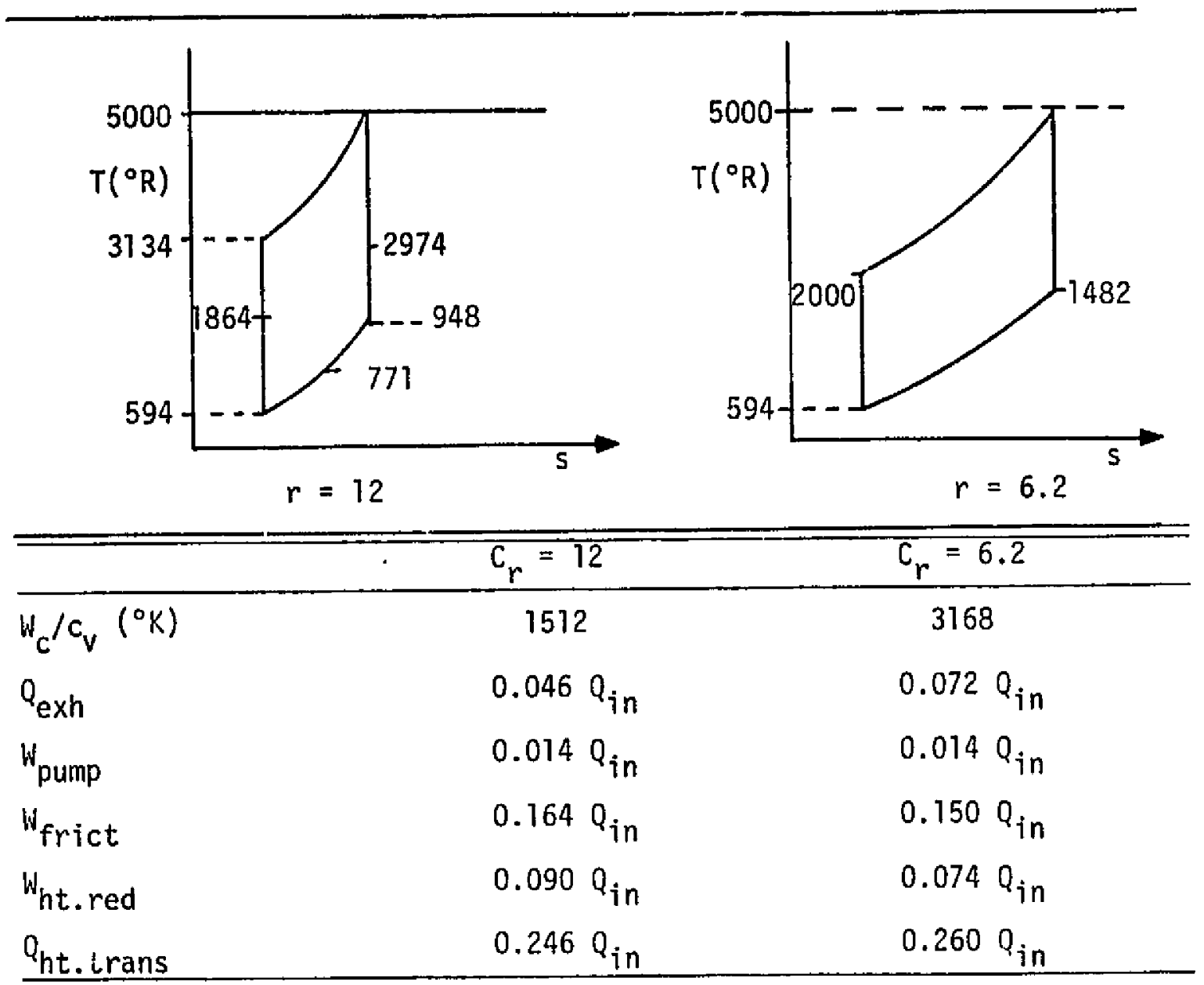

For $r=12, \bar{A}=\frac{A\left(60^{\circ}\right)+A\left(90^{\circ}\right)}{2}=\frac{0.224+0.096}{2}=0.160$

\begin{tabular}{|c|c|c|c|}
\hline PROCESS & HEAT LOSS $\left(\begin{array}{l}0 \\
0\end{array}\right)$ & AVAILABILITY & WORK REDUCTION (S) \\
\hline Compression & 1.2 & 0.16 & 0.19 \\
\hline Heating & 9.5 & 0.81 & 7.70 \\
\hline Expansion & 6.7 & 0.16 & 1.07 \\
\hline \multirow[t]{2}{*}{ Exhaust } & 7.2 & 0.0 & $\underline{0.0}$ \\
\hline & AL $\quad 24.6$ & & 8.96 \\
\hline
\end{tabular}

For $r=12, \eta_{\text {actual }}=\frac{0.81-0.014-0.164-0.090}{1+0.246-0.014-0.164-0.090}=55$ percent 
In contrast with the nonideal thermodynamic cycles, such as the 0tto, Brayton, or Diesel, no advantage can be gained in the Stirling from utilizing a high specific heat ratio fluid. Any gain in efficiency therefore must result from higher working fluid temperatures or a more efficient heat addition process. In the ideal cycle, heat absorption is by isothermal transfer to the working fluid in the cylinder. In the actual cycle, heat addition occurs in heater tubes as the gas passes back and forth between the regenerator and the hot space. In principle, this heater section could be replaced with a chamber of comparable volume, and the laser radiation could be admitted and absorbed through a suitable window. In practice, the advantage of such an innovation awaits the development of high temperature hot space and regenerator components. An additional complication which would be introduced by higher temperature operation is that most of candidate refractory materials have thermal conductivities which are appreciably :Iigher than current stainless steel components. Any increase in the thermal efficiency gained from higher operating temperatures ilould tend to be counterbalanced by the increase in direct heat transfer losses between hot and cold regions of the engine.

Actual performance data for Stirling engines from a recent survey [25] relating efficiency to power on a per cylinder basis are shown in Figure 18. Most of this data is taken from combustion heated Stirling engines. Generally, for small devices, the

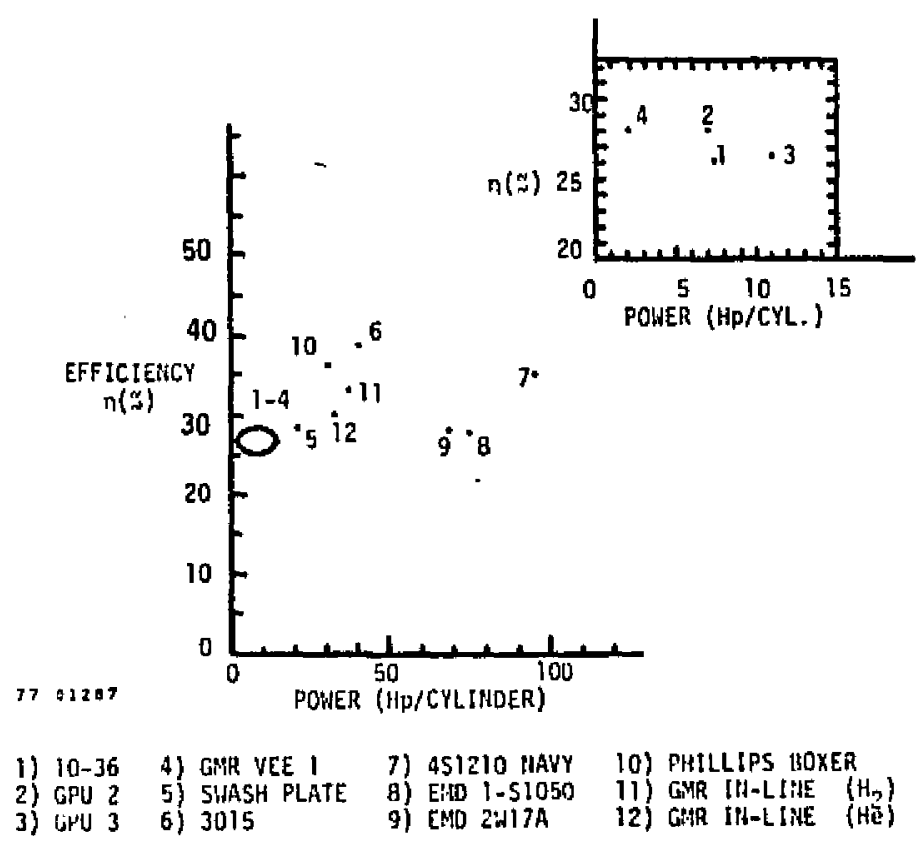

Figure 18. Stirling Engine Test Data for Operating Efficiency Engines (see Ref. 25 for detailed data)

ORIGINAL PAGE IS

OF POOR QUALITY 
efficiency does not exceed 30 percent. The most efficient, complete Stirling engine ran at 39 percent, with 40 horsepower per cylinder. A higher value of efficiency ( 52 percent at 138 horsepower per cylinder) was for coriditions under which combustion gas temperature losses were not properly accounted for; the total engine size was 690 horsepower for this case. It would appear that some gain in efficiency is available by going to larger machines. It is also worth noting that the 39 percent observed efficiency for small engines and 52 percent for large engines refer to no-combustion-loss cases, which probably represent the limits for internally laser heated Stiriing engine efficiencies. The scaling of piston engines with size is discussed in more detail in Section 3.7. Some gain in efficiency may also be obtained by heating with a hot liquid instead of hot combustion gases, since heat transfer would be more effective. This gain can be estimated to be on the order of 5 percent by assuring combustion gas source temperatures of $1370^{\circ} \mathrm{K}$ [25], compared to liquid energy source temperatures of $1050^{\circ} \mathrm{K}$, to obtain Stirling cycle peak temperutures of $1000^{\circ} \mathrm{K}$. Even with this increment, the Stirling cycle efficiencies appear to fall short of the 0tto or Diesel cycle efficiencies estimated previously in this section.

Some attention was given to devising a regenerative cycle using a piston displacer scheme with a laser heat addition spike superimposed. Figure 19 shows TS-PV for such a cycle. This
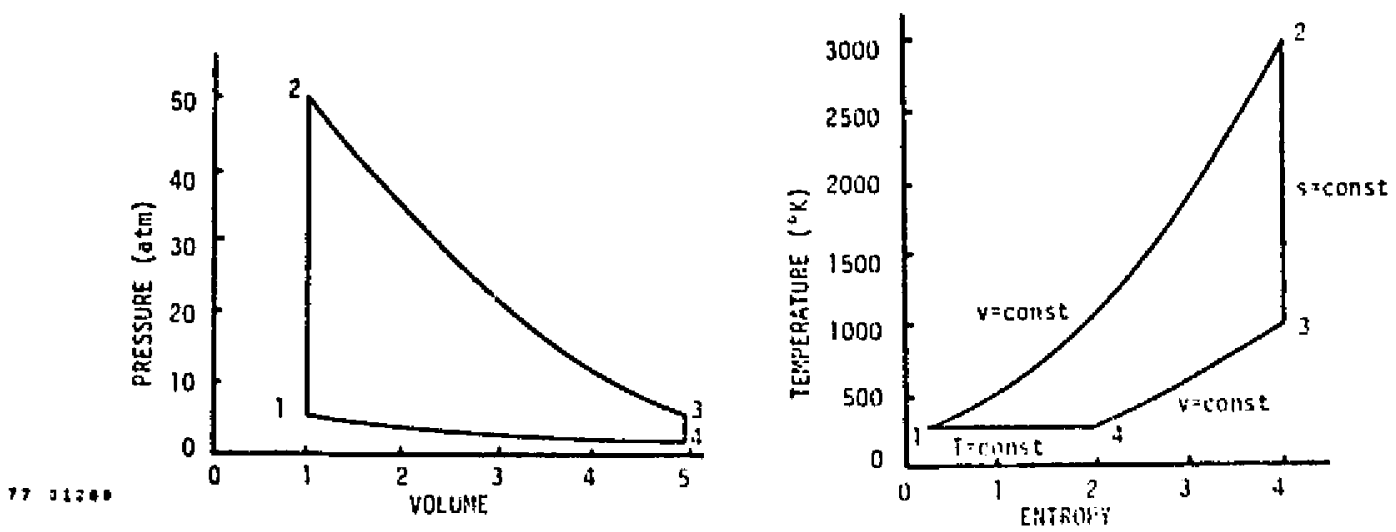

Figure 19. Stirling Cycle with Laser Heat Addition 
scheme has the advantage that it does not require high temperature component development other than the window and hot space absorber section. There are several drawbacks to this approach. The heat conduction problems alluded to earlier are also present in this scheme. The approach also represents a fairly drastic departure from the design envelope of conventional Stirling devices. Nomal Stirling live-to-dead space ratios are on the order of two-to-one. The ratio for this alternate scheme is five-to-one. The performance of such a device is also difficult to predict. The heat transfer and flujd flow processes in the regenerator and heat exchangers are particularly affected by changes in dead space ratio. Extensive developilent would be required to demonstrate the concept.

Extension of the cavity absorption concept to higher efficiencies would also await development of higher temperature components, or demonstration of new Stir]ing technology [7].

In general, for the small size demonstration devices being considered, the 35 percent efficiency (40 to 45 percent engine efficiency), 10-kW, remote power unit represents a limiting efficiency using near-term tecinology.

\subsubsection{MHD Power Generator}

In the conversion of laser radiation to electric power via MHD, the sequence of process steps undergone are the following. The radiation must be absorbed and/or transferred to a gaseous working fluid which rises in temperature to levels where the electron density of the fluid is sufficiently large to achieve conductivities of the order 10 to 100 mhos $/ \mathrm{m}$. The one-dimensional energy equation relating enthalpy gain to radiative power absorbed, electric power removed, and losses incurred is

$$
\rho u \frac{d h_{t}}{d t}=\alpha I+\bar{j} \cdot \bar{E}-\text { losses }
$$

where

$$
\begin{aligned}
& \alpha=\text { absorption coefficient } \\
& I=\text { local radiative intensity }
\end{aligned}
$$




$$
\begin{aligned}
& \bar{j}=\text { current density } \\
& \bar{E}=\text { electric field } \\
& h_{t}=\text { specific total enthalpy }
\end{aligned}
$$

Each of these terms is a power density which may be characterized by a length scale, such as the absorption length and the MHD interaction length.

The absorption length depends primarily on the absorption mechanism, which may be

1) Resonance absorption by the gas

2) Surface absorption on dust particles in the gas

3) Absorption on solid surfaces of the device

4) Inverse bremsstrahlung absorption by free electrons in an alkali metal seed vapor

In the case of resonance absorption, the length scale is determined by the absorber density. Absorption is governed by that portion of the laser radiation spectrum covered by the pressure broadened resonance lines. Absorption on dust is controlled by the dust density and particle size, and enjoys the advantage of absorbing all of the incident radiation it intercepts. Inverse bremsstrahiung absorption length is governed by the collision cross-section and by the electron density.

The absorption of radiative power by these means yields a power input distribution in the direction of the beam which is of an exponential decay type, resulting in high temperature where absorption begins and relatively lower temperature further along. In either class of devices shown in Figure 20, this absorption will lead to spatial temperature nonuniformities, which may be severe enough to degrade the performance of the MHD generator [26].

The MHD interaction length depends on magnetic field strength, gas flow parameters, and the electrical conductivity. The magnetic field strength, $B$, is usually constant at the technically largest value to minimize device size. Gasdynamic parameters are relatively constant when compared to the very strong dependence of conductivity on temperature. 


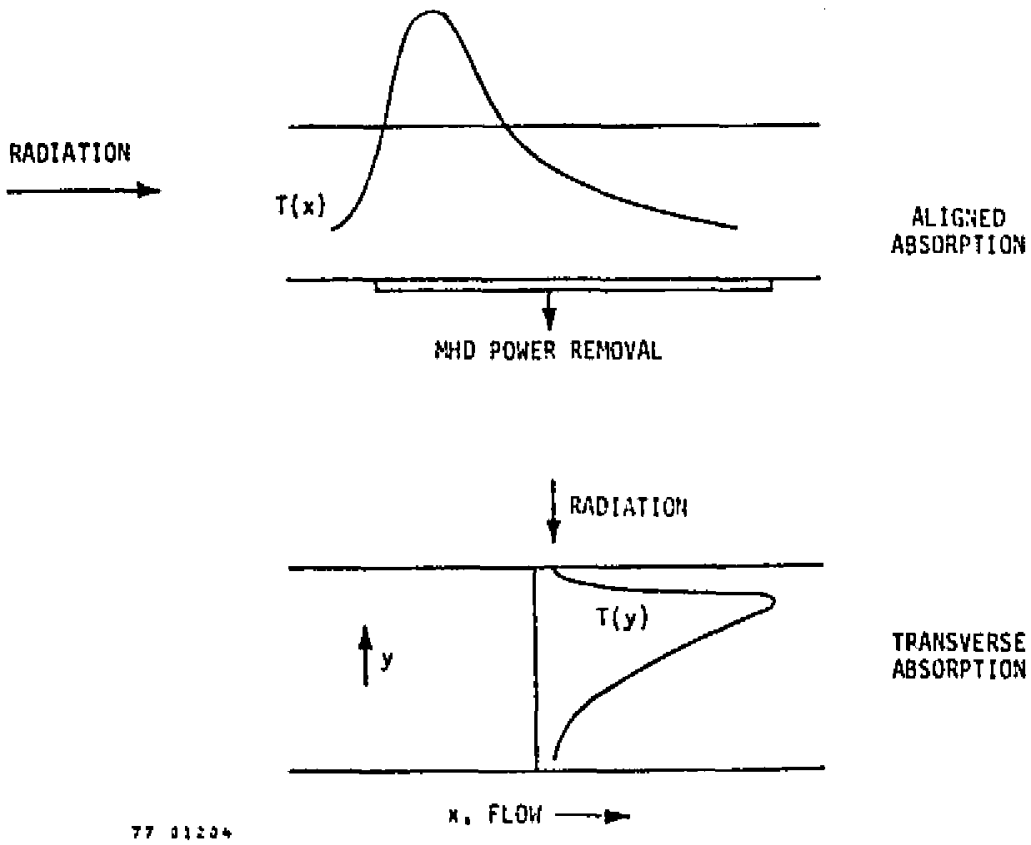

Figure 20. Temperature Nonuniformities in a Laser Heated MHD Channel

Radiation energy sources also offer a unique opportunity to enhance the efficiency of the mechanical-to-electrical conversion by achieving a themodynamic cycle which closely approximates a Carnot cycle. For example, laser radiation can be absorbed down the entire length of an MHD channel to provide nearly isothermal heat addition during the conversion of mechanical-to-electrical energy. While this technique of producing a "hybrid" Carnot cycle has not been fully conceptualized in existing devices, it has sufficient potential for enhancing the thermal efficiency for a given peak temperature that it deserves attention here.

Isothermal absorption requires that the MHD interaction length be constant and that the thermal power input must balance the power required. This is not strictly possible when the absorber is a component of the gas, because the conductivity will vary with the themlal power input to the gas. The best practical means of implementing isothermal absorption is to stage absorption and MHD power removal, as shown in Figure 21. Here, a transverse absorption is used. Tungsten or a similar high temperature material, may be used in an array, as shown in Figure 22, where a diffuser and nozzle between stage elements are used to minimize decay losses associated with the fins. 


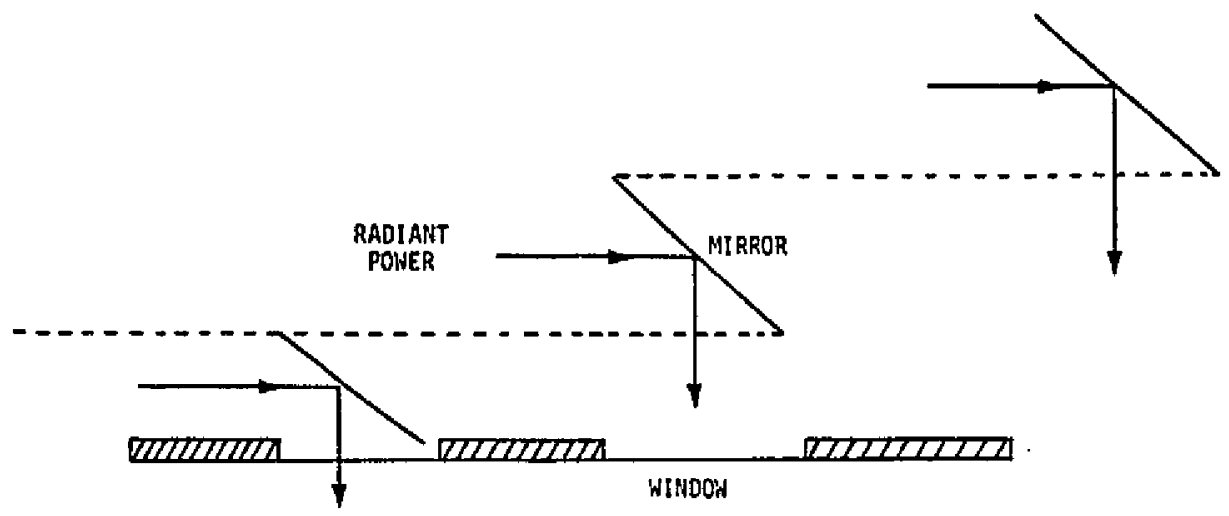

$\stackrel{\text { FLOW }}{\longrightarrow}$

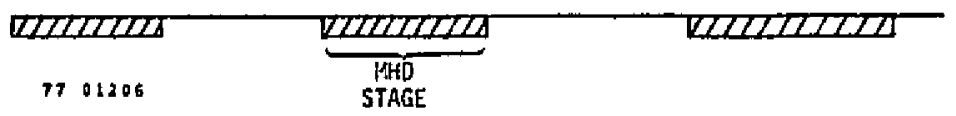

Figure 21. Geometrical Arrangement for Staged Laser Heating of an MHD Channel

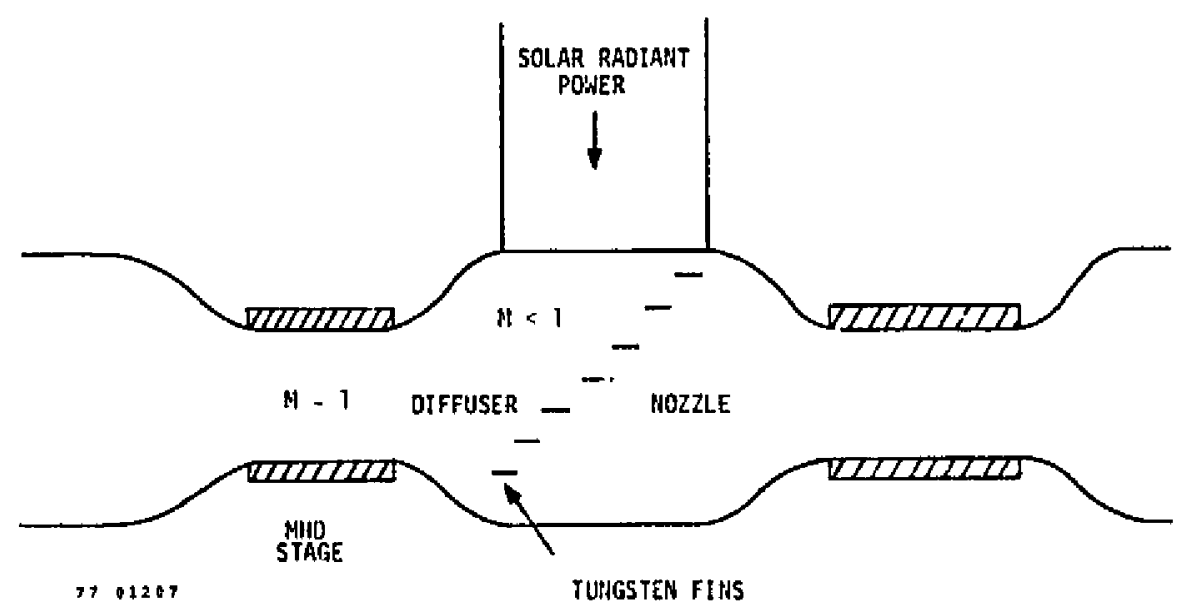

Figure 22. Staged Transverse Converter Using Solid Absorption Surfaces 
Thus, isothermal absorption is approximated by a number of stages, as shown in the Mollier diagram of Figure 23. Typically, the use of three to four such stages offers a reasonable compromise between efficiency and complexity.

A vast body of knowledge exists in which the best MHD systems for use for space power generation were considered looking forward to the day when nuclear reactors operating near $2000^{\circ} \mathrm{K}$ might be available. This effort led to identification of cesium-seeded helium as an attractive medium to be used with an AAHD generator in a closed Brayton cycle. Successful operation of such systems required plasmas with extra thermal ionization to achieve reasonable electrical conductivity. Typically, electron temperatures in the range of 2500 to $3000^{\circ} \mathrm{K}$ are required. If significant enthalpy removal by MHD is to be achieved in a single stage, the stagnation temperature must fall to levels near $2000^{\circ} \mathrm{K}$, and nonequilibrium ionization is required.

The T-S diagram for one stage in the cycle might be as shown in Figure 23. The choice of minimum cycle temperature is somewhat arbitrary, and requires consideration of system weight. Note also that regeneration between the $2000^{\circ} \mathrm{K}$ MHD exhaust and heater inlet

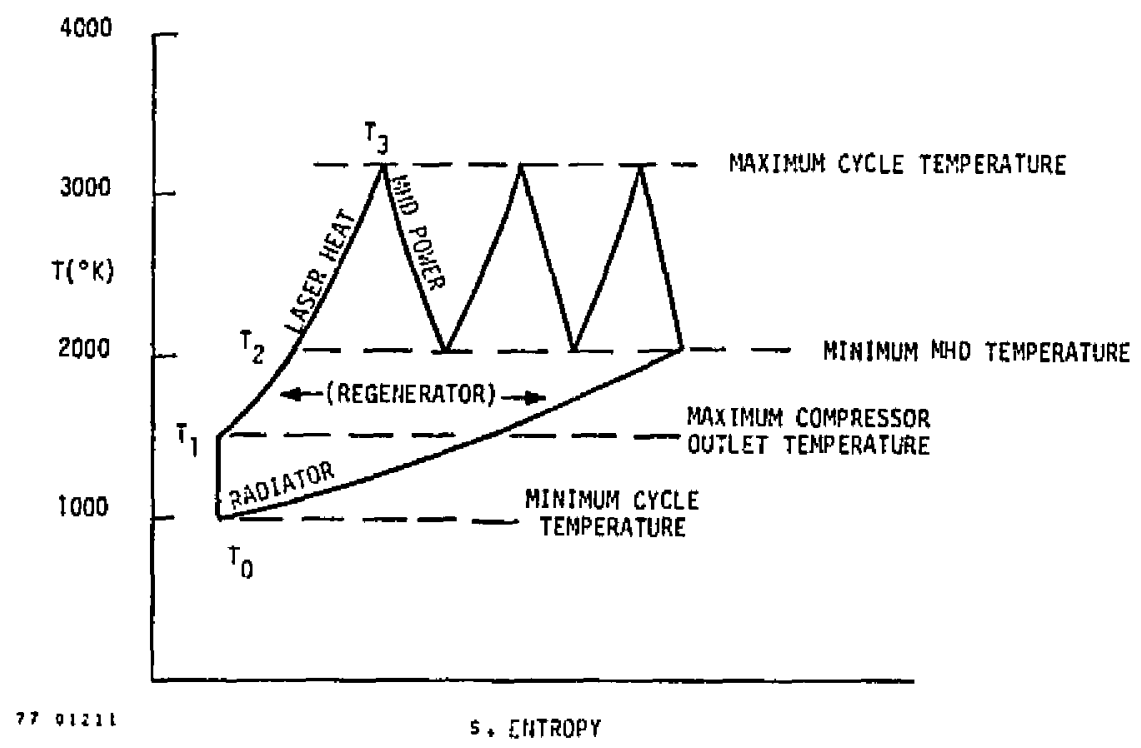

Figure 23. Mollier Diagram for Staged MHD Generator 
requires the use of very high temperature materials. There is no technology in readiress today to accomplish that task. Such a cycle could achieve an enthalpy extraction fraction of $1200 / 3200=38$ percent. The variation of stagnation, static, and electron temperatures through an MHD stage is shown in Figure 24 . Note that near the inlet, the stagnation temperature is greater than the required electron temperature, and near the exit of the generator, it is less. This translates to a more severe electron temperature elevation requirement near the exit.

Electron temperature elevation is possible if the energy input to the electron gas, that is, the joule heating experienced, balances the losses from the gas at an elevated temperature. For
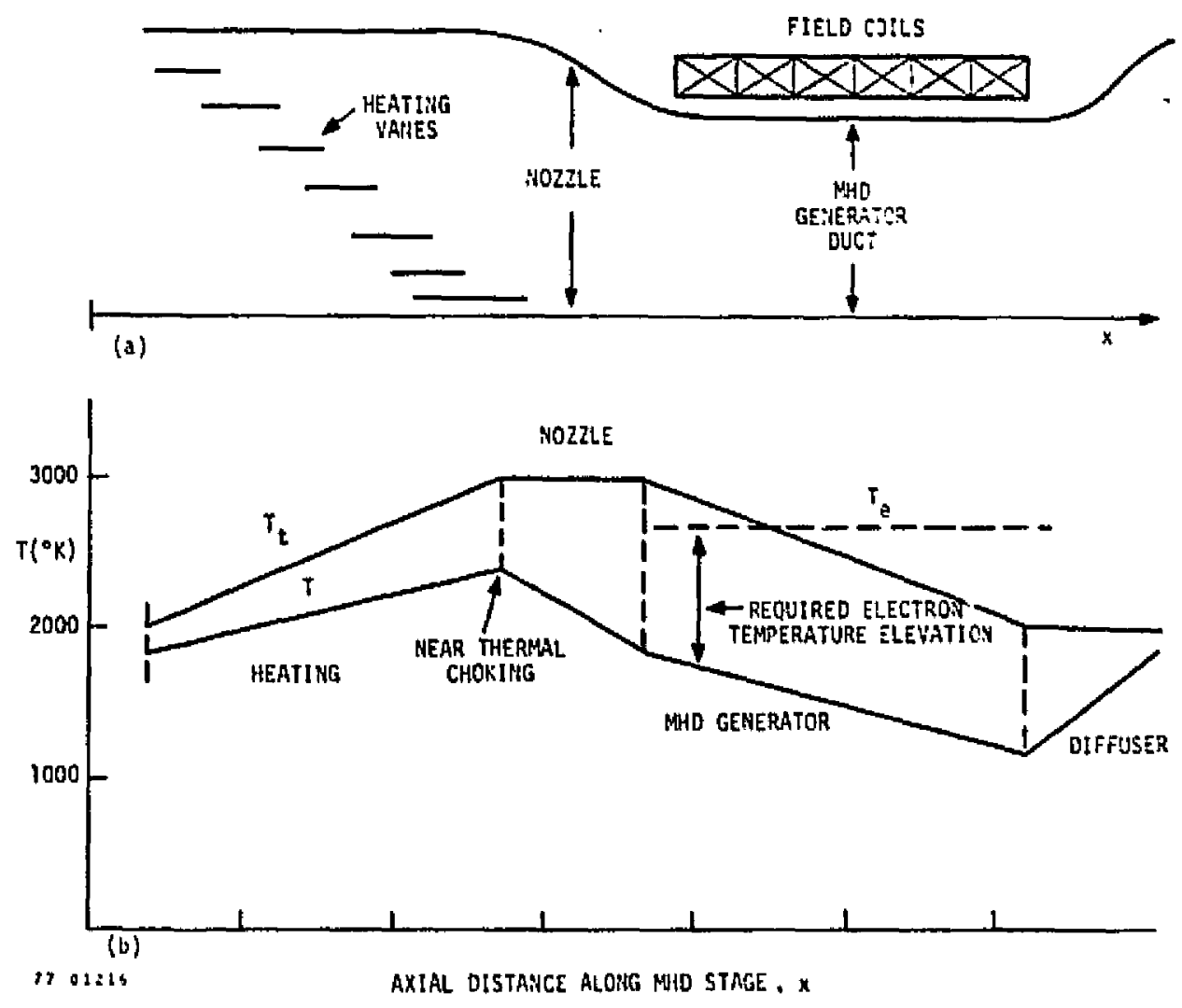

Figure 24. (a) Cross-Section of MHD Duct witi Heating Vanes and Nozzle, and (b) Stagnation and Fluid Temperatures versus Axial Distance 
large-scale devices, the primary electron energy loss is the collisional loss which is proportional to the difference between mean thermal electron and gas energies, and to a parameter characterizing the elasticity of electron-gas atom collisions. With no internal degrees of freedom available, electron collision with noble gases are elastic, and hence small energy transfers occur. Thus, the use of noble gases offers the largest potential for extrathermal ionization. When the electron heating is by $\bar{U} \times \bar{B}$ induced electric fields, the electron temperature is given by

$$
\frac{T_{e}}{T}=1+\frac{5}{9} \beta^{2} M^{2}(1-K)^{2}
$$

where

$$
\begin{aligned}
& T=\text { gas static temperature } \\
& \beta=\text { Hall parameter } \\
& M=\text { flow Mach number } \\
& K=\text { load factor }=E / U B
\end{aligned}
$$

The validity of this relation has been demonstrated in various smal1-scale experiments $[27,28]$, but requires limitation to values of the Hall parameter under two so that wave instabilities cannot grow in the plasma $[29,30]$. One may note here that the load factor, $K$, is a measure of the polytropic efficiency of the generator, so that operation with $K$ close to unity is desirable from the point of view of high efficiency, and detrimental from the point of view of a large electron temperature elevation.

Assuming that the Hall parameter is fixed at a value of two, the load factor achievable may be obtained for various values of $M$ and the ratio of electron to stagnation temperature, $T_{\rho} / T_{\rho}$. Figure 25 shows this relationship. Near the exit of the generator, $T_{0}$ will be on the order of $2000^{\circ} \mathrm{K}$. Reasonable conductivity dictates $T_{e}$ near $2600^{\circ} \mathrm{K}$. Hence, $T_{e} / T_{o} \approx 1.3$, which means that a flow Mach number of 2 is required to obtain a value of $K=0.54$. It is therefore evident that each MHD stage will operate with variable load factor and efficiency; a value near 0.7 may be a more reasonable average value for $K$ to keep the overall efficiency high. 


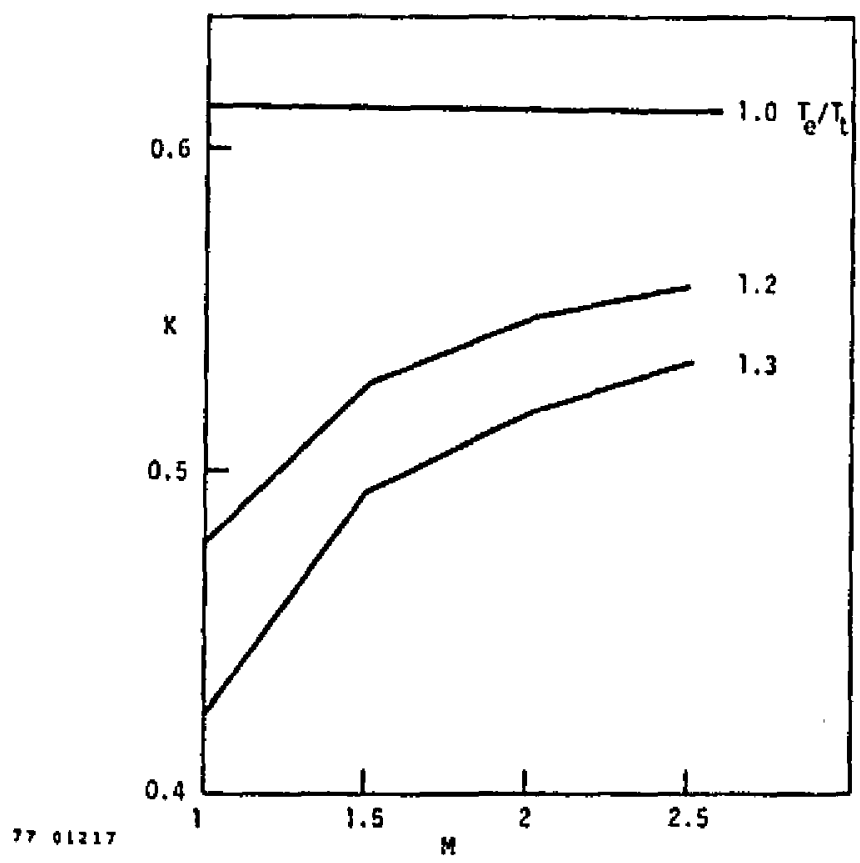

Figure 25. Variation of Maximum Load Factor with Flow Mach Number for Fixed $\beta(=2)$ and $T_{e} / T_{t}$

Size Considerations. With enthalpy transfers of the magnitude considered above, one may calculate the device size required for a given pressure level. For the production of one $k W$ from a single stage operating at a peak pressure of ten atm, the flow area required is such that the duct diameter is on the order of one mint. If the efficiency is only one percent, this diameter increases to one $\mathrm{cm}$. This duct dimension is so small that the boundary layer on the interior surface occupies 40 percent of the flow area. Viscous losses dominate and are large. Viscous losses only become an insignificant fraction of other losses when the size scale approaches that of one MW.

In addition, the electron temperature elevation discussed above requires that joule heating balances only collisional energy losses from the electron gas. In size scales of five $\mathrm{cm}$ or less, resonance radiation of the seed vapor escapes from the electron gas to the walls $[27,31]$ because of the low opacity of the gas.

These two factors predicate against small-scale experiments being realistic as a demonstration for the performance of larger units. Furthermore, the theory required for the extrapolation is complex and possibly inadequate to establish the difference in performance between large and small devices. 
Cycle Efficiency. A preliminary examination of potential cycles using MHD conversion reveals the fact that the temperature ratio $T_{3} / T_{2}$ (as identified in Fig. 23) plays a role similar to the design pressure ratio usually considered in cycle analysis. Thus, with regeneration, cycles with $\mathrm{T}_{3} / \mathrm{T}_{2}$ close to unity give highest efficiency. Larger ratio of $\mathrm{T}_{3} / \mathrm{T}_{2}$ reduces efficiency but increases the specific work, i.e., reduces the size and cost of a system for a given output power Tevel. Note that the specific work for this cycle is

$$
\frac{W}{\grave{n}}=N C_{p}\left(T_{3}-T_{2}\right)-C_{p}\left(T_{1}-T_{0}\right)
$$

For the regenerated cycle shown in Figure 23 with a single stage of compression, the efficiency of the system is shown in Figure 26. Here a polytropic turbine efficiency of 0.7 and a polytropic compressor efficiency of 0.8 were assumed. On this plot, we note that the lowest $T_{3} / T_{2}$ ratio gives highest efficiency, consistent

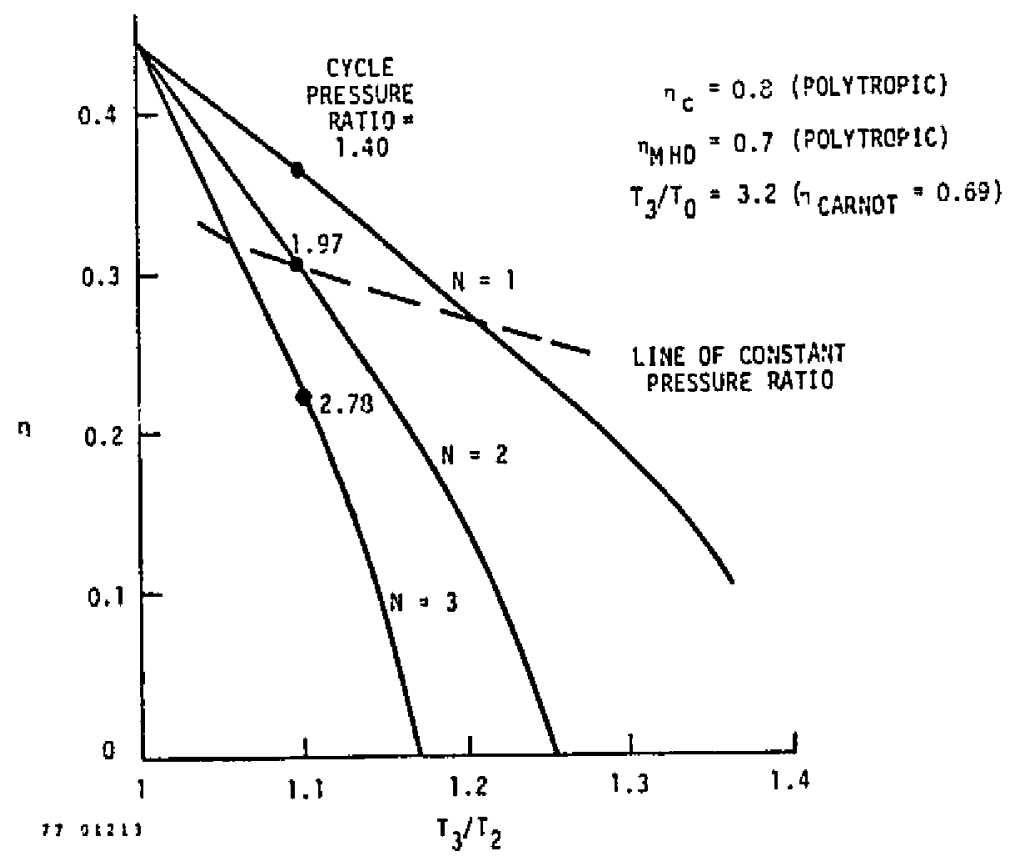

Figure 26. MHD Generator Efficiency vs. Stage Temperature Ratio for it Stages 
with heat input at the highest average temperature. A line of constant cycle pressure ratio is also shown, indicating that for a chosen cycle pressure, the highest efficiency is obtained for the larger number of stages.

The specific work for this cycle is shown in Figure 27 as a function of the $T_{3} / T_{2}$ temperature ratio. Maximum specific work is obtained at modest values of $T_{3} / T_{2}$, indicating that the MHD generator will operate tast with small ratio of power extracted to power recirculated. This ratio is $1-\left(T_{3} / T_{2}\right)^{-1}$, and has values in the neighborhood of 0.1 .

Omission of the regenerator leads to increased heat requirements, which results in reduced efficjency, as shown in Figure 28 (compare to Fig. 27). The cycle without regeneration has the same specific work characteristics as does the regenerated cycle.

Components. The components required to assemble a number of MHD/heater stages cons ist of:

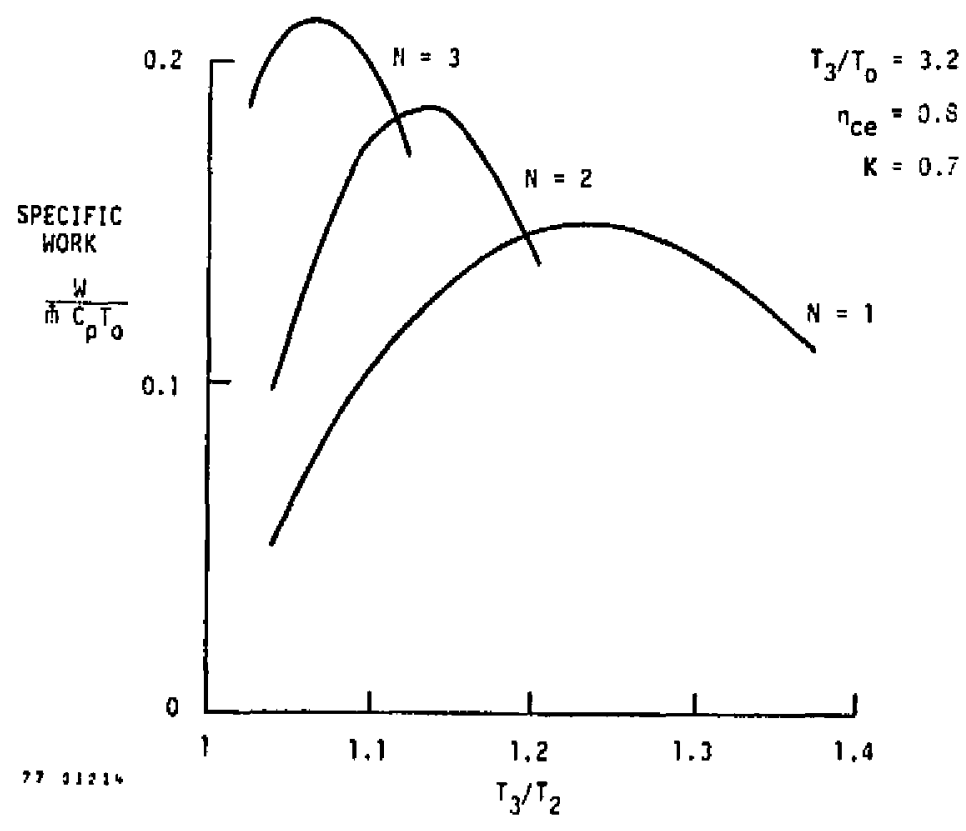

Figure 27. Specific Work vs. Stage Temperature Ratio for a Regenerated Multi-Stage MHD Generator 


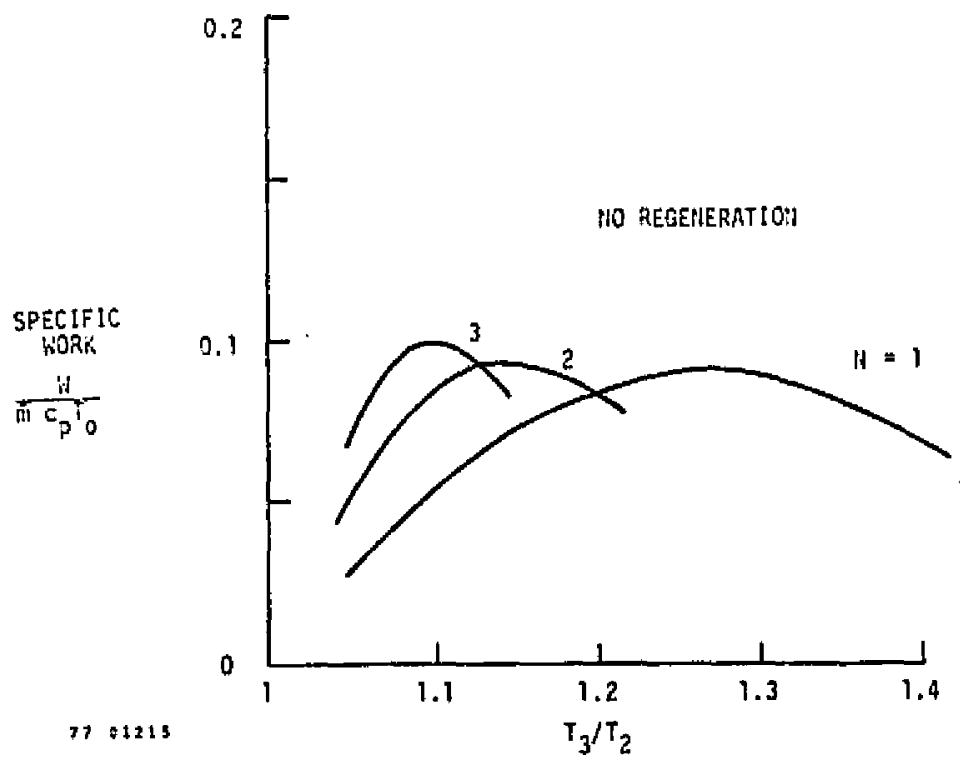

Figure 28. Specific Work vs. Stage Temperature Ratio for MHD Generator' with No Regeneration

1) The MHD generator duct and electrodes.

2) A magnet of sufficient volume to contain the duct. This magnet could be superconducting, in which case makeup power requirements would be smali.

3) Provision ahead and behind the MHD generator to inject and remove alkali metal seed if the cycle reaches low temperatures where condensation may interfere with radiator or compressor operation. In the device discussed here, temperatures may be sufficientiy high that the seed may remain in the gas mixture throughout the cycle. In this case, separate seed handling would be required only for makeup and removal of accumulated seed in portions where buildup may occur.

4) Compressor to close the cycle.

5) A radiator for rejection of thermal power to space.

6) A regenerator capable of operating between 1000 to $3000^{\circ} \mathrm{K}$.

7) Power conditioning equipment to convert MHD generator output to useful form (e.g., AC power).

ORIGINAL PAGE IS
OF POOR QUAIII 
Technology Status. Nearly 15 years of development work have gone into the understanding of the performance limitations of nonequilibrium MHD generators. This work has identified the critical role played by the Hall parameter in the effect of temporal and spatial nonuniformities which are present in the flow. These limitations are particularly difficult to deal with where stagnation temperatures under $2000^{\circ} \mathrm{K}$ are involved. In the work proposed here, temperatures nearer $3000^{\circ} \mathrm{K}$, with a variation to $2700^{\circ} \mathrm{K}$ (approximately 10 percent), are involved. Extrathermal ionization may not be required at all, or if it is, the small electron temperature elevation associated with $\beta \sim 2$ will be adequate. Hence, from the MHD generator point of view, as well as the heat absorber and the regenerator, the difficulty centers around design of high temperature ducts which have to contain the flow and manipulate it. $\mathrm{Clever}$ schemes for adding heat to a conducting gas at $3000^{\circ} \mathrm{K}$ may be required. For example, a solid absorber, such as tungsten, intercepts che entire radiation flux and transfers it to the working medium.

The choice of materials available to handie $3000^{\circ} \mathrm{K}$ gas is obviously limited. The MHD generator, or the regenerator and possibly the heat absorber, have the advantage of operating at relatively low stress levels, hence the demand on the materials is not for strength but for corrosion-resistance in a chemically aggressive environment.

Active cooling widens the list of possible materials. Suitable materials for electrodes (such 2 - graphite-doped refractory oxides, etc.) and for insulators (sucn as boron-nitride, segmented metals, etc.) are available.

In large systems, cooling internal wal is of ducts becomes a smaller penalty, and therefore can be considered for such systems. Such losses scale as the boundary layer thickness $\div$ flow length, which decreases with increasing size.

The chemical properties of hel ium are very benign, and chemical corrosion/reaction problems are limited to the interaction with cesium and any radiation absorbers used in the gas (in preference to a solid absorber). Specific problems associated with corrosion are readily apparent and require appropriate testing of the relevant materials.

Absorption of the laser radiation is also a serious problem in this device. If gas regeneration up to $2000^{\circ} \mathrm{K}$ temperature is possible, then sufficient electron density may be present for inverse bremsstrahlung to occur. This technique has been used successfuliy to ieat plasmas with a $\mathrm{CO}_{2}$ laser at MSNW and elsewhere [32]. However, if regeneration at lower temperatures occurs, 
then there appears to be no simple way to absorb the radiation without incurring large losses through reradiation, as in the use of aosorption on a solid surface.

\subsection{Laser Power Sources}

Among the multitude of lasers that have been discovered in the past 15 years, three gas lasers stand out as candidates for power transfer applications in space. These are: $\mathrm{CO}_{2}$ at $9.2,9.6,10.2$, or $10.6 \mu \mathrm{m}, \mathrm{CO}$ at 5.0 and $5.4 \mu \mathrm{m}$, and $\mathrm{HF}$ at 2.7 to $3.0 \mathrm{\mu m}$. Each of these lasers has been operated at laser power levels in excess of $100 \mathrm{~kW}$, and are scalable in power level over the full range required for space power transfer. Each of these lasers has been operated in both pulsed and $\mathrm{CW}$ modes, and in subsonic and supersonic flow configurations. Generally, both closed and open loop systems are feasible. Each of these lasers has been operated at high electrical efficiency ( 30 to 60 percent for $C 0,20$ to 30 percent for $\mathrm{CO}_{2}$, approximately 100 percent for pulsed $\mathrm{HF}$ ), and low laser gas mass utilization. In the future, it is anticipated that efficient, high average power visible lasers may be developed for this application, but at present, there are none that have been operated at the kilowatt average power level, and none has shown an efficiency that exceeds a few percent.

In the following paragraphs, a brief discussion is given of the principal characteristics of these lasers that need to be considered.

\subsubsection{Wavelength Characteristics}

The wavelength of the laser determines the diffractionlinited beam spread during propagation from source to receiver. The beam spread in turn determines the transmitting and collecting mirror diameters required for efficient power transfer, given the range between sending and receiving stations, as shown, for example, in Section 2.4. If fixed mirror diameters are selected, the laser power received is proportional to $\mathrm{I} / \lambda^{2}$ in the limit of very long distance between source and receiver. Thus, HF would yield about 3.5 times more power than $\mathrm{CO}$, and $\mathrm{CO}$ would yield about 4 times more power than $\mathrm{CO}_{2}$, for the same total laser power emitted from the source for a fixed collector diameter.

A second wavelength issue concerns the output spectrum produced by these lasers. The efficiency of the $\mathrm{CO}_{2}$ laser usualiy is not affected by the number of Iines emitted, and the number of lines can therefore be chosen on the bas is of the requirements of 
the absorber fluid carried in the receiver. On the other hand, $\mathrm{CO}$ lasers are based on partial inversion and cascade coupling of adjacent vibration-rotation bands. At least three, and as many as ten, principal lines are present in the output of an efficient co laser. Similarly, for HF, the bulk of the output appears on five to ten principal lines. Thus, for $\mathrm{CO}$ and $\mathrm{HF}$, the absorber must be capable of absorbing a number of lines; specifically, at least one dominant rotational line from each coniributing vibrational level. At least in the case of $C O$ lasers, there is an important opportunity for high efficiency operation by tailoring both the laser output and the absorbing medium to each other.

\subsubsection{Modes of Operation}

All three of the infrared lasers noted here can be operated efficientiy in either pulsed or CW modes; therefore, the selection can be made on overall power transfer system considerations. In the pulsed mode, the range of pulse durations available is limited and varies from one laser to another as a result of kinetics and the laser operating pressure. For example, $\mathrm{CO}_{2}$ lasers can be operated in a self-sustained mode at short pulse length (i.e., 100 nsec), as externally-sustained discharges (e-beam or (UV) at pulse lengths from 1 to $100 \mu \mathrm{sec}$ (with the longer pulses only for large lasers with many $\mathrm{kJ}$ per pulse), and finally, in a quasi-CW mode with any pulse length by tuning a CW power supply on and off appropriately. Co laser pulses can further be controlled by changing the gas injxture; 5 to $10 \mu \mathrm{sec}$ pulses have been obtained at MSNW in a cryogenic pulsed e-beam system while much longer pulses, greater than 100 usec, have been produced in nitrogen containing mixtures at Northrop. Long laser pulses in electric discharge lasers, particularly if good, stable beam quality is required, are frequently limited by the so-called cathode wava, a shock wave traversing the laser cavity created by the energy deposited $i_{i}$ the cathode fall region. Thus, small lasers have shorter pulse lengths. Pulsed HF chemical lasers have pulses on the order of 5 to $10 \mu \mathrm{sec}$. However, the state-of-the-art of pulsed chemical lasers is still very elementa'y and they are not a serious contender.

The laser optical system must provide a diffraction-limited beam. Tr: is can be achieved either by an oscillator/amplifier configuration, by an unstable resonator configuration, or by a specially designed stable resonator configuration. We will assume that a diffraction-limited laser source can be provided at any of the three wavelength regions noted above. 
The demonstration will probably require a laser power in the range of 10 to $50 \mathrm{~kW}$. For example, a 50 percent efficient, 1-kilowatt output piston engine will require a laser input power during the heating pulse of

$$
P_{L}=\frac{1 \mathrm{~kW}}{(0.5)}\left(\frac{\tau_{\text {cycle }}}{\tau_{\text {heat }}}\right)
$$

where $\tau_{\text {heat }} / \tau_{\text {cycle }}=\alpha$ is the fraction of the engis:e cycle devoted to laser heat addition; i.e., the heating duty cycle. For an otto cycle, $\alpha \leqslant 0.05$, requiring $\mathrm{PL}_{L} \geq 40 \mathrm{~kW}$ and for a Diesel cycle, $\alpha \approx 0.15$ requiring $P_{L} \cong 13 \mathrm{~kW}$.

The heating pulse length, $\tau_{L}$, needed to drive a piston engine also depends on the heating duty cycle and on the rpm as follows:

$$
\tau_{L}(\text { secs })=\alpha\left(\frac{60}{r p m}\right)
$$

Thus, for the example above, $\tau_{L}$ (0tto cycle) $\leqslant 6 \mathrm{msec}$ and $\tau_{L}($ Diesel $)=18 \mathrm{msec}$. These pulse lengths can be achieved by chopping a CW laser beam or by manufacturing a pulsed laser wi th the appropriate characteristics. In the otto cycle example, the laser pulse can be considerably shorter than the limit computed above because a constant volume heat addition is required. By contrast, the Diesel cycle employs a constant pressure heat addition so that rather little deviation from the laser pulse length shown above is al lowed. Lower liml to the laser pulse length are imposed if the energy per cycle is dolivered in too short a time. Then the instantaneous power may be high enough to "bleach" the absorbers and makp the gas transparent so that not enough energy can be absorbed. Bleaching limits are computed in Section 2.6.

From a practical point of view, only the $\mathrm{CO}_{2}$ laser is avajlable today in the power ranges and run durations required for the near-ten demonstration project. Carbon dioxide lasers in the 1 to $10 \mathrm{~kW}$ range are commercially avallable (at a cost of approximately $\$ 40$ to $\$ 50 /$ watt) from Photon Sources, CILAS, AVC0, GTE Sylvania, BOC Ltd., and United Technologies. MSNW has also built and operated a number of lasers in this class. A somewhat larger $\mathrm{CO}_{2}$ laser, in the 50-kW size range, exists within NASA at the Ames Research Center; the Lewis Research Laboratory has a closed cycle $\mathrm{CO} 2$ EDL operating at a power level of $15 \mathrm{~kW}$. Purchase, rental or construction of a $\mathrm{CO}_{2}$ laser $\mathrm{fc}$. use in the demonstration program would not be a serious 
technical problem, but the costs may be too high. Table 7 shows a summary of available large $\mathrm{CO}_{2}$ lasers and their status.

Few CO lasers exist in this performance range, particularly truly $\mathrm{CW}$ devices. Much development of CO laser technology has been performed in a quasi-CW (milliseconds) mode. MSNW has been a leader in the development of supersonic $C 0$ lasers operating in this quasisteady mode (Ludwieg tube operation). We have produced over 100-kW powers and over 37 percent efficiencies, however for run times of the order of milliseconds. The Boeing Company has a high power, pulsed $\mathrm{CO}$ laser that has a run time of 10 msecs. Calspan has a 1-kW supersonic expansion $C O$ laser that has a run time of 50 secs. Garrett/AiResearch has a 1- to 10-kW, cooled, subsonic $\mathrm{CO}$ laser that has operated for 15 secs. In most of these cases, the run times are too short for meaningful experiments. Further, for the $\mathrm{CO}$ laser, a problem exists in correctly matching the multiline output with the absorption spectrum of the working fluid (see Section 2.5). This problem would require modification of the existing Co laser facilities or construction of new facllities, for any optimized experiment. Consequently, the CO laser is considered as a longer range option, not for the early demonstrations. Table 8 is a summary of the status of available co lasers with the necessary high power capability.

Few HF lasers exist in the 1- to 10-kW range of average output power. There is a $1-\mathrm{kW}$ supersonic mixing, continuous wave HF laser at Aerospace Corporation. TRW has a number of high power, supersonic mixing, continuous wave HF lasers with output powers of tens of kilowatts. Pulsed HF lasers are available commercially from Lumonics Ltd.; however, they do not produce high enough average output to be used in this demonstration program. Generally, HF lasers are unsatisfactory from the point of view of requiring substantial new chemical energy supplies to the laser. Closed loop HF Taser operation does not seem feasible at present, and so the overall system aspects of developing a laser heat engine to work with an HF laser appear initially unattractive. Table 8 also includes a sumary of the available HF lasers and their status.

\subsection{Collector/Concentrator}

The basic properties of optical transmitting and receiving antennas [33] are well known; however, we shall briefly review these properties in order to direct our attention to the particular problems or constraints imposed on the engine concepts uncer consideration. 
Table 7

Existing High Power $\mathrm{CO}_{2}$ Lasers

\begin{tabular}{|c|c|c|c|}
\hline $\mathrm{CO}_{2}$ LASERS & POWER (kW) & RUN TIME & STATUS \\
\hline NASA-Ames (GDL) & 50 & seconds & operational \\
\hline NASA-Lewis (EDL) & 15 & hours & operational \\
\hline P\&W-Florida (GDL) & $>200$ & seconds & $\begin{array}{r}\text { operationa } \\
(\text { military })\end{array}$ \\
\hline $\begin{array}{l}\text { AVCO/Everett } \\
\text { Humdinger (EDL) }\end{array}$ & $>200$ (pulsed) & seconds & $\begin{array}{c}\text { operational? } \\
\text { (military) }\end{array}$ \\
\hline Thumper (EDL) & $>200 \quad$ (pulsed) & seconds & $\begin{array}{c}\text { operational } \\
(m \neq 1 \text { tary })\end{array}$ \\
\hline $\begin{array}{l}\text { Hughes } \\
\quad \text { Peacemaker (EDL) }\end{array}$ & $>200$ & -- & $\begin{array}{l}\text { operational } \\
\text { (military) }\end{array}$ \\
\hline MIT/Lincoln Lab (EDL) & pulsed & hours & deveTopment \\
\hline USAF/Kirtland (GDL) & $>200$ & seconds & $\begin{array}{l}\text { uperational } \\
\text { (military) }\end{array}$ \\
\hline GTE/Sylvania & 5 & hours & $\begin{array}{l}\text { commercial } \\
\$ 200 \mathrm{~K}\end{array}$ \\
\hline AVCO/HPL- 15 & 15 & hours & $\begin{array}{l}\text { commercial } \\
\$ 500 \mathrm{~K}\end{array}$ \\
\hline UTL & 10 & hours & $\begin{array}{l}\text { commercial } \\
\$ 400 \mathrm{~K}\end{array}$ \\
\hline
\end{tabular}


Table 8

Existing High Power $\mathrm{CO}$ and HF/DF Lasers

\begin{tabular}{|c|c|c|c|}
\hline CO LASERS (EDL) & POWER (KW) & RUN TIME & STATUS \\
\hline $\begin{array}{l}\text { Northrup } \\
\text { Big Shot }\end{array}$ & $>200$ & msecs & non-operational \\
\hline Ecole & $>200$ & minutes & $\begin{array}{l}\text { deve lopment } \\
\text { (military) }\end{array}$ \\
\hline $\begin{array}{l}\text { Boeing } \\
\text { Ludwieg Tube }\end{array}$ & $>100$ & msecs & non-operationa] \\
\hline $\begin{array}{l}\text { MSNW } \\
\text { Ludwieg Tube }\end{array}$ & 100 & msecs & non-operational \\
\hline Calspan & 1 & minutes & operational \\
\hline Garrett/AiResearch & 10 & seconds & non-operational \\
\hline \multicolumn{4}{|l|}{$H F / D F$ LASERS (CL) } \\
\hline $\begin{array}{l}\text { TRW } \\
\text { NACL }\end{array}$ & $>200$ & seconds & $\begin{array}{c}\text { operational } \\
\text { (military) }\end{array}$ \\
\hline Miracle & $>200$ & seconds & $\begin{array}{c}\text { non-operational } \\
(\text { military) }\end{array}$ \\
\hline
\end{tabular}




\subsubsection{Transmitter and Receiver Mirror Dimensions}

The minimum size of the transmitter and receiver mirrors is constrained by the diffraction properties of the transmitter optics. A beam of uniform intensity will have a half-width in the far field (measured to the first minimum) given by

$$
\text { half-width }=1.22 \lambda R / 0_{t}
$$

where $R$ is the range between the transmitter and receiver, $\lambda$ the wavelength, and $D_{t}$ the diameter of the transmitter mirror. If the receiver mirror is constructed to have a diameter, $D_{c}$, equal to the full far-field beam width, we will collect 84 percent of the transmitted energy,

$$
D_{c} \equiv \frac{2.44 \times R}{D_{t}}
$$

To illustrate the relative size of $D_{c}$ and $D_{t}$, this equation has been plotted in Figure 29 . The ranges for a low earth orbit $(185 \mathrm{~km})$ and a geostationary orbit $(38,000 \mathrm{~km})$ have been used, along with wavelengths of 5.0 and 10.6 microns.

It is clear from Figure 29 that for a geostationary orbit, the transmitter and receiver mirrors must be approximately 15 and 30 meters in diameter at 5.0 and 10.6 microns, respectively. One can, of course, pick a smaller transmitter mirror at the expense of a larger receiver mirror, and vice versa. Just what relative sizes should be chosen is not inmediately clear, as it depends on several other factors, including:

1) Obtaining the optimum intensity profile in the far field. This includes picking an appropriate configuration and near-field intensity profile.

2) Weight restrictions.

3) Maximum intensity limits on the optical eleinents.

\section{ORIGINAL PAGE IS
OF POOR QUALITY}




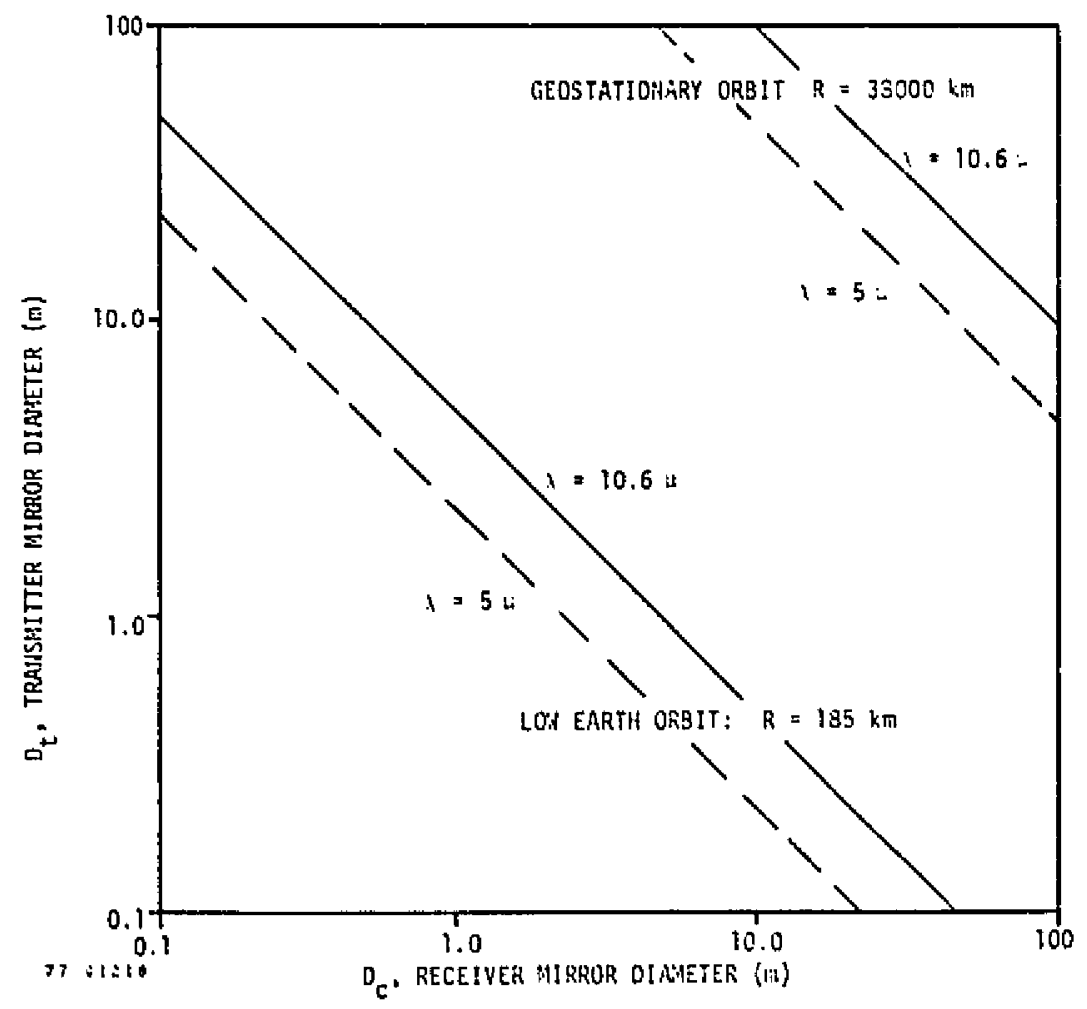

Figure 29. Transmitter Mirror Diameter, $D_{t}$, versus Receiver Mirror Diameter, $D_{C}$, for Various Wavelengths and Ranges

4) Cost trade-off between constructing a small, "high quality" transmitter inirror and a large, "poor quality" collecting mirror versus a large, "high quality" transmitter mirror and a small, "poor quality" collecting mirror.

To be more precise, in regard to item 4 , we should comment that a transmitter optical system that generates a wavefront with a RMS deviation of only $\lambda / 14$ from an ideal spherical wavefront will cause a 20 percent drop in the far-field peak intensity. Associated with 
such a reduction will be a loss in the total energy received by the collection optics. Thus, by "high quality" mirror, we mean one that introduces less than a $\lambda / 14$ RMS deviation in the beam wavefront. On the other hand, the receiver mirror does not require such severe tolerances because the beam does not need to be focussed to a diffraction-limited spot. We shall consider what tolerances are required of the collection mirror in a later section. As a consequence, it may be less costly overall to construct a transmitting mirror that is smaller than the receiver mirror. In regard to item 1, one should note that the far-field intensity profile for a variety of near-field beam intensity distributions have been considered by a host of investigators. In particular, Nored [34] reports that NASA-Lewis has studied the encircled energy for gaussian and uniform beams of the same total power. Their results indicate that a gaussian beam truncated at its $\mathrm{e}^{-2}$ intensity point maximizes the amount of power into a collector of a given size. Recentiy, the general problem of finding an optimum intensity profile for an optical system to achieve a desired intensity distribution over a given receiving plane, or to maximize the energy into a given area, has been solved analytically by Vero and Asakura [35]. Their results can be exploited to find the most desirable intensity profile for a given telescope aperture. In addition, they remark that the addition of aberrations in the near field can never lead to a higher degree of coliected energy in the far field for any intensity profile [36]. Therefore, we are led to the conclusion that aberrations must be removed from the transmitted beam to achieve an acceptable efficiency.

If the laser output beam has a sufficiently good phase front, and the beam is to be used outside the earth's atmosphere, we can assure a diffraction-limited beam by simply constructing a sufficiently good telescope to expand the laser beam.

If the atmosphere must be negotiated, and/or the telescope optics cannot be constructed with the desired tolerances, and/or the laser output wavefront is of poor quality, one is forced to use an adaptive optics scheme to correct for these effects. The coherent optical adaptive techniques [37] (COAT) has other advantages: it greatly eases the pointing and tracking requirements for the transmitter mirror mounting system.

\subsubsection{Receiver: Optical Design}

As mentioned earlier, the shape of the receiver mirror need not be of high optical quality because a diffraction-limited focal spot is not required. The maximun size of the focal spot is limited only by the window diameter on the absorption cell. 
The variety of collector optics can be grouped into two categories which we call imaging and non-imaging systems. The nonimaging systems would be similar to many types of solar energy collector-concentrators. These systems could be attractive, since they may reduce the requirements on pointing accuracy; however, the size of these collectors and the degree of concentration required will tend to be quite large. Furthermore, they do not strongly control the direction of the collected beam as it enters the engine absorption volume. Because of this, we will restrict our attention, at present, to imaging systems.

\subsubsection{Single El ement Collectors}

The single element collector consists of a receiving mirror with the engine absorption cell located near the focal point. The mirror diameter wili be specified by the range to the transmitter, the transmitting mirror diameter, the beam quality, and the wavelength. Once the diameter is fixed by these parameters, we have only the focal length and the quality of the receiving mirror surface to specify. This can be accomplished by considering the constraints imposed by:

1) Our ability to accurately point the receiving system towards the incoming beam.

2) The physical size of the window at the absorption cell.

3) The physical size of the entire collectorabsorption cell system.

The pointing accuracy required can be estimated by defining the maximum angular deviation, $\alpha$, to be

$$
\alpha \leq \frac{D_{W}}{8 f}
$$

where $D_{w}$ is the absorption cell window diameter and $f$ is the collection mirror focal lenyth. 
The optical quality of the mirror surface can also be specified in terms of the absorption cell window diameter, $\mathrm{D}_{W}$. Here we require that the diameter of the blur circle, caused by geometric aberrations of the mirror surface, to be less than the absorption cell window diameter. Only geometrical effects are considered, since the diffraction spot size is several orders of magnitude smaller than any reasonable window diameter. In order to calculate representative blur circle diameters, and to estimate the degree of mirror deformation that is allowable, we consider a specific mirror deformation and compare it with an ideal parabolic mirror. The two mirror surfaces are described by the following equations.

$$
\begin{gathered}
Y_{\text {parabolic }}=\frac{x^{2}}{4 f} \\
Y_{\text {aberrated }}=\frac{x^{2}}{4 f}+a x^{4}+b x^{6}+\ldots
\end{gathered}
$$

By specifying the constants $a, b, c, \ldots$, we specify the magnitude of radialiy symmetric surface discortions measured from a parabola (in this case, we have neglected rocal shifts that go like $x^{2}$ ). For simplicity, we consider only the first non-parabolic term, $\mathrm{ax}^{4}$, which causes third-order spherical aberration. The deformation, $\delta$, of the mirror at its edge is just

$$
\delta \equiv Y_{\text {aberrated }}\left(D_{c} / 2\right)-Y_{\text {parabolic }}\left(D_{c} / 2\right)=a \frac{D_{c}^{4}}{16}
$$

where $D_{C}$ is the diameter of the collection mirror. It is easy to show that the blur diameter for this aberration at the gaussian image plane is given as

$$
\text { blur diameter }=2 \mathrm{aD}_{\mathrm{c}}^{4} f
$$

The minimum blur diameter is found not to be at the gaussian image plane, but at a distance $3 / 4$ (LSA) from this plane. LSA is the longitudinal spherical aberration. 
At this position, the minimum blur diameter is $1 / n$ of the result given in Equation (5). Using this result, we can set a 7 imit on the absorption cell window diameter as

$$
D_{w} \geq \frac{a D_{c}^{3} f}{2}
$$

Using Equations (4), (5) and (6), we obtain the desired relationships,

$$
\begin{gathered}
D_{W} \geq 8 f\left(\delta / D_{C}\right) \\
\alpha \leq \delta / D_{C}
\end{gathered}
$$

Then, Equations (7) and (8) are plotted (using an equality sign) in Figure 30 to illustrate the limitations placed on the mirror focal length once values of $D_{C}, D_{w,}$, and $\alpha$ are fixed.

From Figure 30 , we note that as we increase $\delta / D_{C}$ and hold the focal length fixed, the minimum window diameter increases as expected. Holding $\delta / D_{C}$ fixed and increasing the focal length a Tso requires an increase in the window diameter; this is because the angular error of the rays remains fixed while the observation distance increases.

The laser engine absorption cells being considered for this program are quite small (a few cubic inches); as an example, we can pick the corresponding values of $\delta / D_{C}$ and $f$ by moving along the line plotted for a $3-\mathrm{cm}$ diameter window. Table 9 tabulates the relevant parameters for two systems:

1) Low earth orbit

2) Geostationary orbit.

The tolerances on the mirrors seem rather severe. One should recall, however, that a good optical mirror would have a maximum edge deformation, $s$, of less thar $\lambda / 4$, or a $\delta / D_{c}$ for a $30-m$ micror of only $8.3 \times 10^{-8}$ with $\lambda=10.6 \mu$. Parabolic collectors 


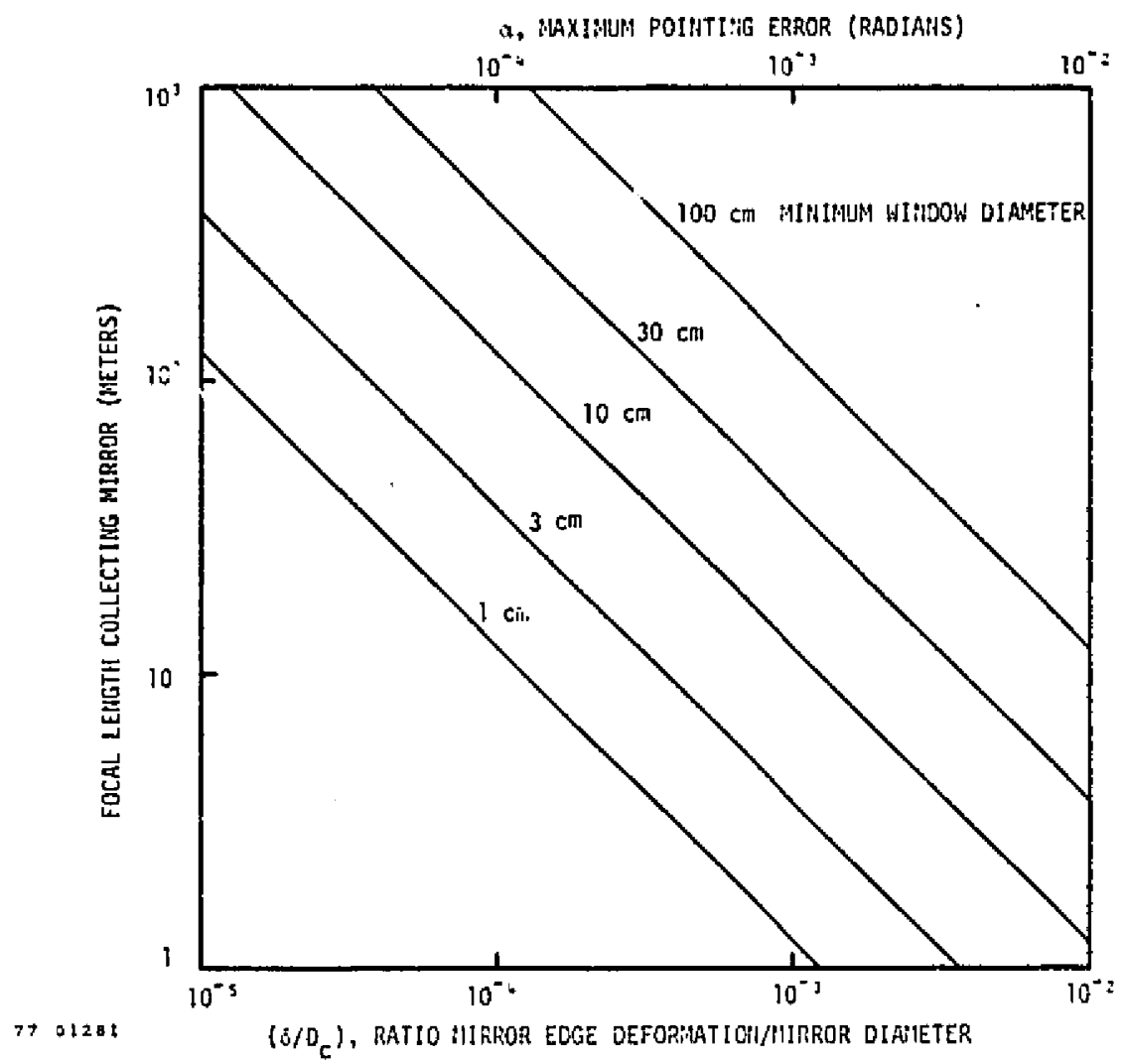

Figure 30. Mirror Deformation and Pointing Error Limits as Functions of Focal Length and Window Diameters

for solar-powered heat engines were constructed at NASA-Lewis Research Center in 1967 with $\left(\delta / D_{C}\right)=3 \times 10^{-4}$, where the mirror diameter was 12 meters [38]. 
Table 9

Relevant Parameters for Low Earth and Geostationary Orbits

$$
(\lambda=10.6 \mu)
$$

\begin{tabular}{|c|c|c|c|c|}
\hline CASE 1 & \multicolumn{4}{|c|}{$\begin{array}{ll}\text { Low Earth Orbit: } & \text { Range }=185 \mathrm{~km} \\
& D_{c}=D_{t}=2.0 \mathrm{~m} \\
& D_{w}=3.0 \mathrm{~cm}\end{array}$} \\
\hline $\mathrm{F} / \#$ & $f(m)$ & $\delta / D_{c}$ & $\delta(\mathrm{cm})$ & $\alpha \leq$ (mrad) \\
\hline 1 & 2 & $2 \times 10^{-3}$ & 0.4 & 2.0 \\
\hline 2 & 4 & $1 \times 10^{-3}$ & 0.2 & 1.0 \\
\hline CASE 2 & \multicolumn{4}{|c|}{$\begin{array}{ll}\text { Geostationary Orbit: } & \text { Range }=38,000 \mathrm{~km} \\
& D_{c}=D_{t}=30 \mathrm{~m} \\
& D_{w}^{c}=3.0 \mathrm{~cm}\end{array}$} \\
\hline$F / \#$ & $f(m)$ & $\delta / D_{c}$ & $\$(\mathrm{~cm})$ & $\alpha \leq$ (mrad) \\
\hline 1 & 30 & $1.2 \times 10^{-4}$ & 0.36 & 0.12 \\
\hline 2 & 60 & $6 \times 10^{-5}$ & 0.18 & 0.06 \\
\hline
\end{tabular}

\subsection{Receiving Windows}

Previous discussions of the laser heat engine window problems have been conducted on the conceptual level, and several promising design ideas have emerged $[5,7]$. Constraints on possible window materials arising from optical, thermal, and stress considerations have also been discussed. In light of this previous work, a detailed examination of the window problem was performed, and various solutions were evaluated on the basis of probable performances under realistic design conditions. Of the window concepts examined, the double window scheme proposed by Garbuny and Pechersky [7] with actively cooled inner faces is a particularly attractive approach having general applicability.

Two important conclusions emerged from the detailed study of this window design. The first is that even under the most severe operating conditions, thermal stresses within the window material can be kept small, and secondly, the side in contact with the working fluid can be maintained at any desired temperature with a minimal investment in coolant pumping power. The basis for these conclusions and their engineering design implications are discussed below. 


\section{5.?. Double Window Configuration}

The double window mounting scheme is shown in Figure 31. This approach will work well in both piston engine or continuous wave application. The ma in features include exterior and interior gas-tight seals, and a perforated spacer, which separates the windows without severely restricting the coolant flow. The seals may be plated with ductile material, and should protrude slightiy above the seafing groove to approximate a simply supported edge condition.

Although a clamped edge leads to lower stress per unit thickness than does a simpiy supported condition, in practice the latter configuration is frequently preferred. This is particularly true in the case of crystalline window materials where local clamping stresses might produce cracking. The simply supported configuration has an additional advantage in that in the presence of a 1 inear temperature gradient, across the thickness, there is no thermal stress.

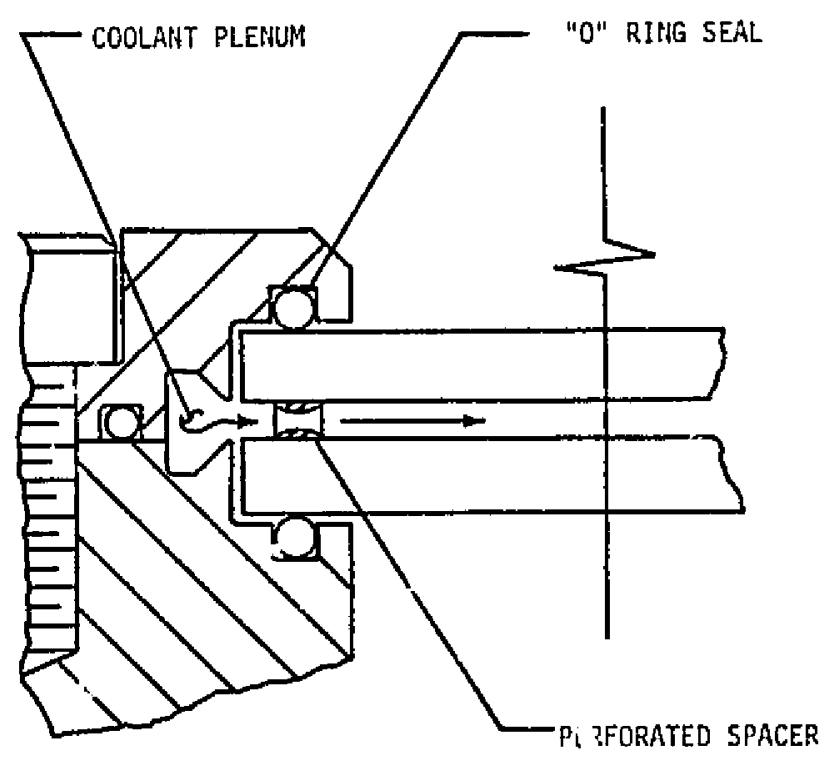

7701290

Figure 31. Face-Cooled Double Window Mounting Configuration

ORIGINAL PAGT IS

OF POOR QUALITI 


\subsubsection{Thermal Stresses}

The most severe thermal stress condition occurs at the startup of either a pulsed or a CW device. The magnitude of the surface tumperature excursion and the accompanying tinermal stress can be obtained from the following considerations.

The temperature profile in the wall and the boundary layer fr.llowing a gas temperature excursion are governed by the unsteady heat conduction equation:

$$
\frac{\partial T}{\partial t}=-k \frac{\partial^{2} T}{\partial x^{2}}=+\frac{\partial}{\partial x} \Phi
$$

where

$$
\phi=-k \frac{\partial T}{\partial x} \cong h\left(T_{g}-T_{w}\right)
$$

and

$$
\Delta T \cong \frac{\Delta t \Phi}{\Delta x}
$$

is the temperature excursion at the window surface. The heat wave penetration distance into the window in time $\Delta t$ is

$$
\Delta x=\sqrt{\alpha \Delta \bar{t}}
$$

where $\alpha$ is the thermal diffusivity. Then, representing $T_{W_{0}}$ as the temperature average in the window material,

$$
T_{W}-T_{w_{0}}=h\left(T_{g}-T_{w}\right) \sqrt{\Delta t / \alpha}
$$

where $T_{w}$ is the instantaneous tempe. ature of the window surface. Let

$$
B=h \sqrt{d t / c}
$$


then,

$$
T_{w}-T_{w_{0}}=\frac{\beta\left(T_{g}+T_{w_{0}}\right)}{1-\beta}
$$

A boundary layer heat transfer coefficint of the order of 5 to 50 $W / \mathrm{m}^{2}{ }^{\circ} \mathrm{K}$ is a representative value for the conductance likely to be found in either pulsed or CW devices. A pulse duration of 0.025 seconds is characteristic of a piston engire compression heat addition time. Table 10 gives the properties of various window materials, including values corresponding to conditions given above.

\section{Table 10}

Mechanical and Thermal Properties of Various

Window Materials (taken from Refs. 39,65 )

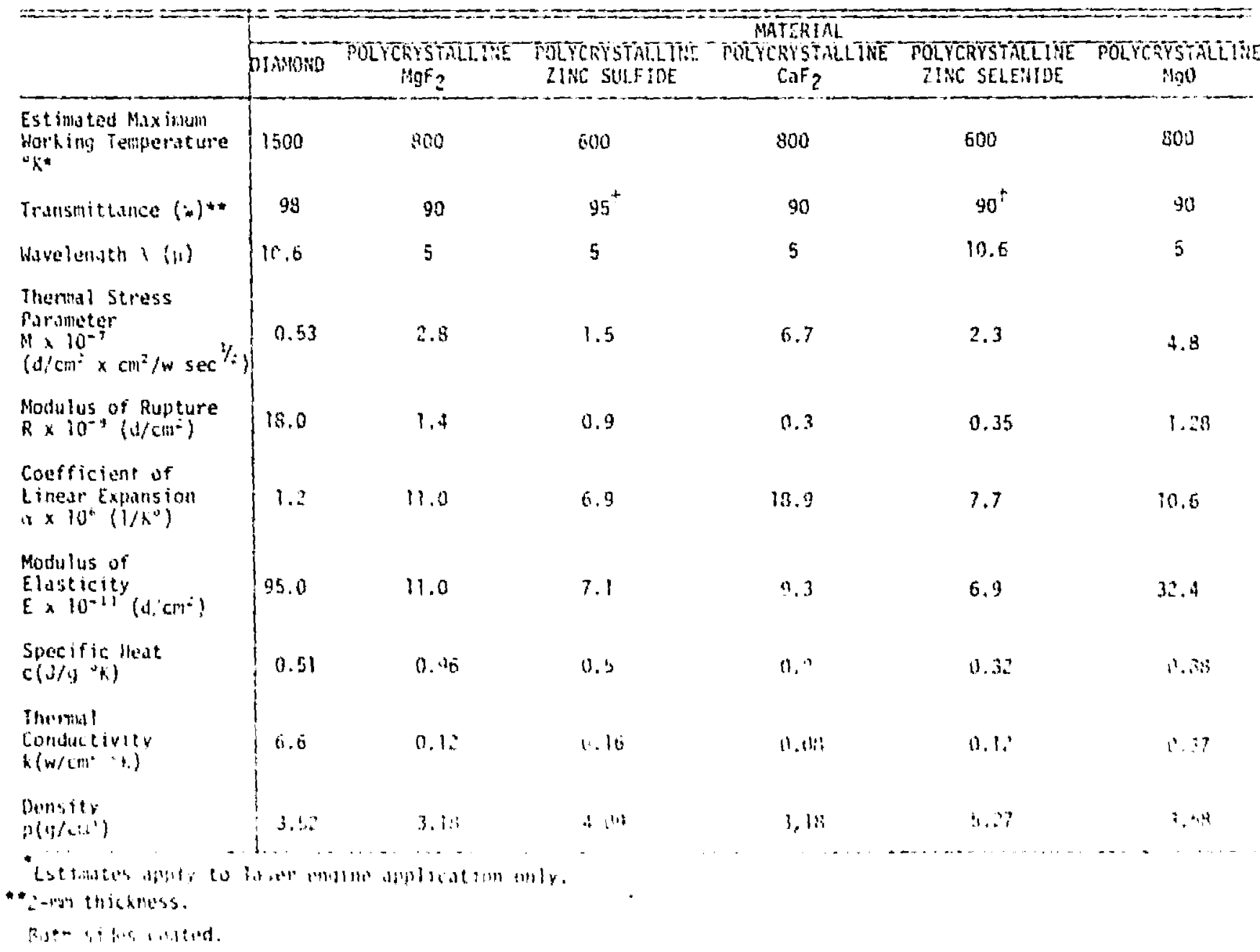


The temperature excursions in the surface of a typical window material, like polycrystalline $\mathrm{MgF}_{2}$, with an initial temperature of $300^{\circ} \mathrm{K}$ and a gas temperature jump of $2700^{\circ} \mathrm{K}$, is

$$
\Delta T=\frac{0.003(3000+300)}{1-0.003}=9.9{ }^{\circ} \mathrm{K}
$$

The expression for the resulting thermal stress is

$$
\sigma_{\text {th }}=\frac{\alpha E^{*}}{T-v}\left(\frac{4}{\pi \rho p^{k}}\right)^{1 / 2} \cdot \Delta t^{1 / 2}=M s t^{1 / 2}
$$

Table 10 also gives the themal stress parameter, $M$, for each material. For $\operatorname{ligF}_{2}$, the stress is

$$
\begin{gathered}
\sigma_{\text {th }}=\frac{2.8 \times 10^{7}}{0.7}(0.006)(3000-300)(0.025)^{1 / 2} \\
=6.8 \times 10^{7} \mathrm{~d} / \mathrm{cm}^{2}(1020 \mathrm{ps} i)
\end{gathered}
$$

Since this is always compressive stress and window materials are much stronger in compression than tension, the thermal stress in this representative case is small. The materials selected for Table 10 are the result of a more widespread survey of suitable window materials conducted for this study, in which other materials have been eliminated because of low temperature limits, low strength, lower optical transmittance at the desired wavelengths, and/or because of chemical incompatibilities with working fluid/absorber combinations.

\subsubsection{Pi*essure Stresses}

The maximum stress for a simply supported circular disk with a uniformly distributed pressure load is

$$
\therefore=1.24 \frac{\mathrm{pr}^{2}}{\mathrm{t}^{2}}
$$

where $r$ is the plate radius, $t$ is the thickness, and $p$ is the appljed pressure. A safety of four is consistent with engineering 
design practice for window material. For $\mathrm{MgF}_{2}$, Table 10 gives the modulus of rupture as

$$
R=1.4 \times 10^{9} \frac{\mathrm{d}}{\mathrm{cm}^{2}}(21,000 \mathrm{psi})
$$

For a 20-atm differential across a single window, the diameter to thickness ratio would be

$$
\frac{\mathrm{D}}{\mathrm{t}}=\sqrt{\mathrm{R} / 1.24 \mathrm{p}}=7.5
$$

indicating that for engine operating conditions, reasonable window thicknesses can be expected with normal window materials.

\subsection{Radiation Absorbers and Working Fluids}

\subsubsection{Absorption Mechanisms and Working Fluids}

Once the radiation has been collected and focussed, the energy must be absorbed and converted to thermal energy in the working fluid. There are two types of conversion processes that can be considered. The first involves the absorption of laser radiation by a gaseous absorbing specie distributed throughout the working gas and the subsequent thermalization of that energy to provide a volume heat addition to the working gas. The second involves the thermal absorption of the laser radiation by a solid and subsequent heat transfer to the working gas. Because of temperature limitations on solid heat exchangers (e.g., $F_{\max }=\left(100^{\circ} \mathrm{K}\right)$ this second approach has not been considered further.

In the first conversion process, the laser energy is absorbed in the volume of the working gas. Two absorption processes fit into this category: inverse bremsstrahlung and molecular resonance absorption. The laser energy is converted directly to translational energy of the working gas through inverse bremsstrahlung absorption. The absorption process is also spectrally continuous so that there is no specific laser wavelength required. However, the presence of free electrons is required and the absorption coefficient is therefore very small in an equilibrium gas at low temperatures. The addition of an easily ionized seed such as cesium would raise the absorption to the point that the process might be viable in a high temperature topping cycle. For operation at lower temperatures, some means of nonequilibrium ionization must be provided. This could be accomplished by an electric discharge or by the production of local gas breakdown with a focussed laser beam. Generally, inverse bremistrahlung appears sufficiently more complicated compared to resonance absorption that it has been considered only in the case of advanced engine concepts such as MHD generators where very high temperatures are required. 
Resonance absorption of laser radiation into the vibrationalrotational bands of molecular gases requires an absorber gas with a resonance coincident with the wavelength of a high power laser. The potential exists for heating to very high maximum cycle temperatures using this process since the equivalent blackbody temperature of the laser is very high. If its absorption cross section is large, the absorber gas may form only a small portion of the total working gas, thus allowing the properties of the working fluid to be chosen in a manner that optimizes the overall cycle efficiency. If the absorption is from the ground state of a molecule, the process can be efficient in a cold gas. Several diatomic gases have large absorption cross sections at wavelengths provided by high power lasers. For example, calculations by Caledonia have shown that NO has a $0-1$ vibrational level absorption cross section of approximately $5 \times 10^{-20} \mathrm{~cm}^{2}$ at the wavelength of severai CO laser lines near 5.3 microns [42].

There are several limitations to this process however, imposed by thermal saturation, bleaching, and dissociation. Also, the absorbing molecules exhibit large cross sections over limited wavelength ranges, thus equiring accurately matched lasers. Each of these limitations is described below and solutions are presented.

Thermal saturation decreases the absorption coefficient with rising temperature as the upper level of the absorbing molecule, which is in equilibrium at the translational temperature of the working gas, becomes highly populated. Thermal saturation occurs at a characteristic temperature $\mathrm{TS}_{S}=h \mathrm{~h} / \mathrm{k \lambda}$ at which the absorption coefficient has decreased enough to seriously affect engine performance. However, hot bands, which involve absorption from lower states above the ground state, exhibit an absorption maximum at $T=T_{S}$. This effect, which counters thermal saturation, can be utilized in the case of a $\mathrm{CO}$ absorber. Since the ground state transition is very difficult to obtain in a co laser, a higher transition can be used to drive the hot band absorption in co molecules that possess appreciable population of the appropriate lower level. Detajled absorption calculations for $\mathrm{CO}$ are presented below in Section 2.6.2.

Bleaching is a nonequilibrium phenomenon similar to thermal saturation. It can occur in a gas with a relatively low translational temperature and is dependent upon the rate of energy deposition in the absorbing gas. When the upper and lower level populations in the absorbing gas are equal (for equal degeneracy values), absorption vanisnes. This can occur if absorption takes place in a time that is short compared to the therma 1 relaxation time of the levels. The bleaching phenomenon establishes an upper limit to the rate that energy can be punped into the absorber (i.e., the absorbed power). The primary concern is in the case of cyclic laser engines. It has been shown that to avoid bleaching, the laser pulse length should be greater than twice the thermal relaxation time in the gas [5]. This thermal relaxation time is considerably shortened in the presence of a light, monatomic gas such as helium, which is also a very good working fluid in a cyclic engine. 
Dissociation of the absorber gas at the maximum cycle temperature will remove absorbing molecules and reduce the absorption coefficient. In addition, the resulting constituents may chemically react with their surroundings before they are able to recombine. This would result in the contamination of the working gas, the corrosion of the engine materials, and the degradation of the absorbing medium. The extent of dissociation is related to the heat of formation of the molecule. This heat can be expressed as a characteristic temperature for dissociation $T_{0}=$ $\Delta h / k$. Since the maximum temperature in the cycle will approximate the thermal saturation temperature of the absorbing gas, the importance of dissociation in a cycle can be assessed from the ratio To/Ts. This ratio is given for several absorbing gases below:

\begin{tabular}{|c|c|c|c|c|}
\hline Absorber & Wavelength (micron) & $\mathrm{T}_{\mathrm{S}}\left({ }^{\circ} \mathrm{K}\right)$ & $\mathrm{T}_{\mathrm{D}}\left({ }^{\circ} \mathrm{K}\right)$ & $\mathrm{T}_{\mathrm{O}} / \mathrm{T}_{\mathrm{S}}$ \\
\hline $\begin{array}{l}\mathrm{D}_{2} \mathrm{O} \\
\mathrm{SF}_{6} \\
\mathrm{NO} \\
\mathrm{CO} \\
\mathrm{HCl} \\
\mathrm{HF}\end{array}$ & $\begin{array}{r}10.6 \\
10.6 \\
5.2 \\
4.7 \\
3.5 \\
2.8\end{array}$ & $\begin{array}{l}1380 \\
1380 \\
2740 \\
3100 \\
4100 \\
5100\end{array}$ & $\begin{array}{r}33,550 \\
36,300 \\
75,500 \\
129,000 \\
51,400 \\
68,500\end{array}$ & $\begin{array}{l}24 \\
26 \\
28 \\
42 \\
13 \\
13\end{array}$ \\
\hline
\end{tabular}

Both $\mathrm{NO}$ and $\mathrm{CO}$ represent good absorbers for $\mathrm{CO}$ laser radiation. It is very difficult to operate a $\mathrm{CO}$ laser on its 1-0 vibrational transition at 4.7 microns, however. The 5.2-micron wavejength represents a readijy obtainable 8-7 vibrational transition. The table above gives only an indication of the potential for problems with dissociation of the absorber.

The final limitation on resonance absorption process, imposed by the finite bandwidth of the absorbing molecules, can be eased by pressure broadening. Absorption linewidths of diatomic gases depend upon the pressure. Higher pressure raises the collision frequency of the absorbing molecule which increases the linewidth of a collisionally-broadened spectrum. When the pressure is high enough, the linewidth exceeds the line spacing and continuous absorption occurs over the entire rotational band, representing a given vibrational transition. This effect not only allows more freedom in the matching of the laser and absorber but also minimizes the effects of themal saturation and bleaching [42]. This discussion has presented several aspects of the absorption and energy transfer process that must be examined when the choice of a suitable absorber is being considered for each thermodynamic cycle.

The best working fluids are gases with high values of the ratio of specific heats. These gases are structurally uncomplicated and the largest fraction of absorbed laser energy appears as translational energy which can be converted to pressure work during the cycle. This effect can be seen in the expression for the efficiency of the cold gas standard otto cycle, $n=T-r^{1-k}$, where for the same fixed compression ratio, $r$, of 8 , a diatomic working fluid $(k=1.40)$ gives an efficiency of 56 percent and a monatomic working gas $(y=1.67)$ 
gives 75 percent efficiency. For example, the laser heated engine can utilize a monatomic gas such as helium that is volumetricaliy heated through a dilute dispersed absorber, such as NO; the gas mixture has a high ratio of specific heats. Helium is very efficient in vibrational deexcitation and is not adversely affected by high temperatures, retaining its chemical stability and inertness. This example illustrates how well suited the jaser heating concept is to the case in which an otherwise efficient engine cycle is limited by materials constraints to low peak cycle temperature (e.g., Stirling, Ericsson cycles). With additional materiali development, these cycles may approach the maximum efficiency possible within their temperature constraints. To boost cycle efficiencies further, the energy exchanger can be utilized to deliver available work from a very high temperature gas that has been heated by laser absorption to conventional cycle hardware at a lower temperature level. The overall system efficiency will be determined by the high temperature of the absorbing gas.

\subsubsection{Absorption of Radiation from CO Lasers}

CO lasers produce infrared radiation on several lines near 5 microns. Any absorbing molecule therefore has a characteristic vibrational temberature near $2900^{\circ} \mathrm{K}$, thus providing appreciable absorption up to the high temperature levels that allow efficient heat engine operation. Furthermore, there is considerable data on the spectral output capability of high powered c0 lasers, as described elsewhere in this report. For these reasons, a search was undertaken for a suitable absorber molecule of radiation from a $\mathrm{CO}$ laser.

There are two basic types of high power co lasers that could supply power to the heat engine: the cooled subsonic flowing laser and the supersonic flowing laser. The emission spectra of these lasers can differ somewhat. Measurements of fractional output power appearing on the emission lines of two existing laser devices representing these types are plotted against output wavelength in Figure 32 . The data for the subsonic flow laser were measured for a low pressure longitudinal discharge laser cooled with liquid nitrogen at Northrop [43], and will be assumed to be typical of this class of lasers. The data for the supersonic flow laser were measured for a Mach 4 pre-excited laser at Calspan [44]. These spectral distributions span the range of wavelength typically available in co lasers. The specifi: output spectrum is very sensitive to temperatures of the gas mixture and to pumping rate. The data show that although two of the subsonic lines contain more than 20 percent each of the total power, the supersonic laser lines are much more numerous and extend to shorter wavelengths. The two intense lines of the subsonic laser are 7-6 P16 and 9-8 P14 and the shortest wavelength emitted is 5.12 microns. The power is more evenly 


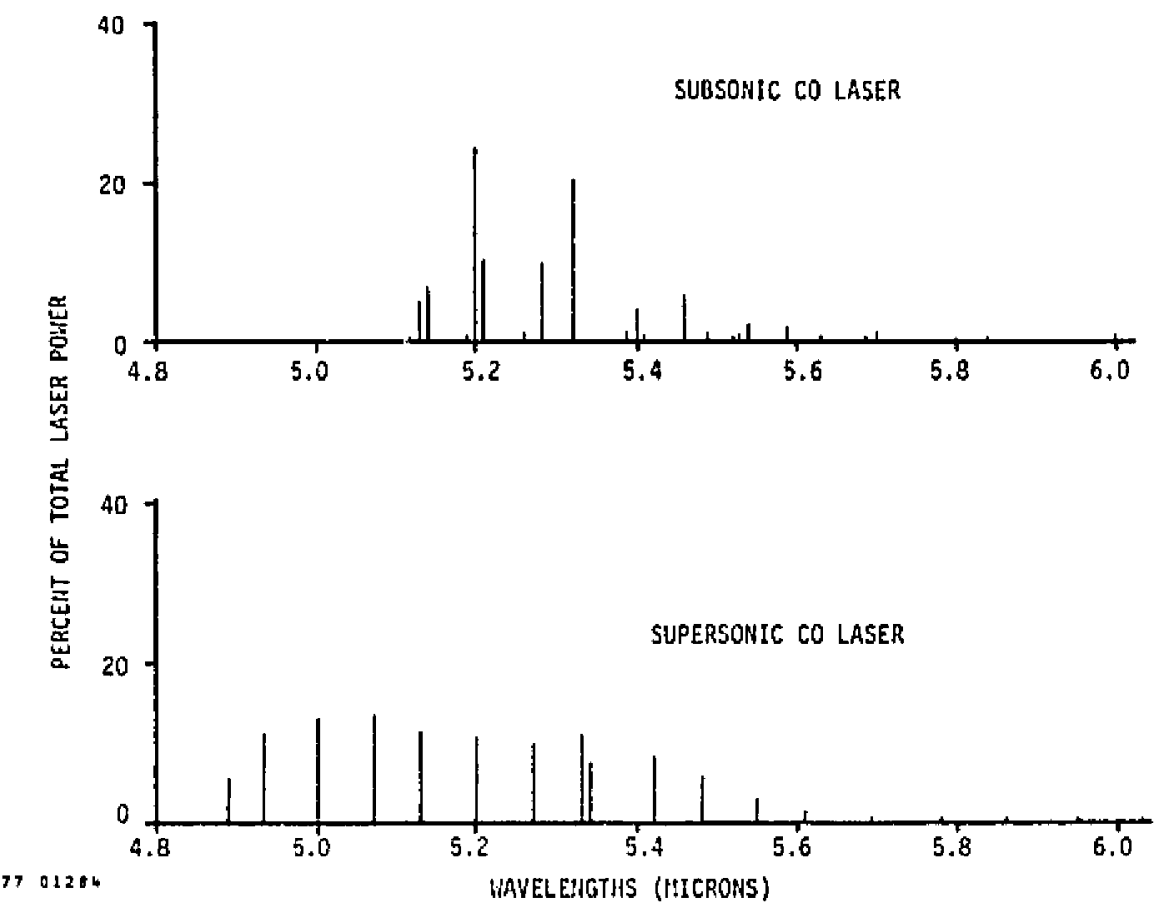

Figure 32. Output Spectra of Typical Co Lasers

distributed over the emission lines from the supersonic laser with a maximum near 5 microns (4-3 P10), and power emitted at wavelengths as short as 4.9 microns.

The absorber gas must be chosen with appreciable absorption on as many laser lines as possible. The process of resonant infrared absorption involves the elevation of molecules from one vibrational energy state to a higher vibrational energy state. The energy difference between these two states is equal to the energy of the infrared photon absorbed in the process. The absorber molecule must possess energy level separations equivalent to the wavelengths of the laser used. In addition, the lower level from which absorption occurs must be well populated in the absorber molecule. The use of $\mathrm{CO}$ as an absorber gas fulfills the prior requi ement well since it possesses the same energy levels as the lasing gas. At temperatures below $1000^{\circ} \mathrm{K}$, however, the high vibrational levels that serve as reservoirs for absorption are not well populated and the absorption coefficient is very small. During the initial phases of the heat input portion of the engine cycle, the temperature of the absorbing medium will be less than $1000^{\circ} \mathrm{K}$ and the use of $\mathrm{CO}$ as an absorber would not allow adequate absorption at such 
temperatures. An absorbing molecule that exhibits resonance with the 1 aser radiator (near 5-microns wavelength) for transitions from lowlying vibrational Tevels is needed. The above spectral outputs will be used to show that such matching is possible.

Absorption calculations have shown that NO is well suited to the absorption of radiation from co lasers, especialiy at high pressures where the absorption lines are pressure broadened to a linewidth comparable to the line separation. Preliminary calculations [45] showed that a mixture of one perceni $\ldots$ in the monatomic working fluid of the heat engine would provide enough absorption to allow deposition of the laser energy in a reasunably sized absorber volume. However, the question of thermal stability of the NO molecule at the high cycle temperatures anticipated has not been adequately considered. In the following discussicn, a system of chemical reactions is described for the decomposition of NO at high temperatures. The equilibrium concentration of NO is a 99 percent inert gas bath at $2700^{\circ} \mathrm{K}$ and 20 atms total pressure is calculated. Since this concentration is found to be very smal1, the rate equations are solved to determine the overall rati of decomposition of the NO. This rate is compared with a typical cycle rate and conclusions are drawn concerning the use of NO as an absorber in such a high temperature laser heat engine.

The system of chemical reactions describing the decomposition of NO have been derived in Reference 46 . The four most important reactions are:

$$
\begin{aligned}
& \mathrm{NO}+\mathrm{NO}=\mathrm{N}_{2} \mathrm{O}+\mathrm{O} \\
& \mathrm{NO}+\mathrm{O}=\mathrm{O}_{2}+\mathrm{N} \\
& \mathrm{NO}+\mathrm{N}=\mathrm{N}_{2}+0 \\
& \mathrm{O}+\mathrm{N}_{2} \mathrm{O}=\mathrm{O}_{2}+\mathrm{N}_{2}
\end{aligned}
$$

Reaction (a) provides the initial 0 atom concentration that starts the chain reaction represented by reactions (b) and (c), which deplete the concentration of NO. Reaction (d) represents the major loss process for the 0 atoms. The reaction rates at $2700^{\circ} \mathrm{K}$ were calculated from the data given in Reference 46 . 


$$
\begin{aligned}
& k_{1}=1.4 \times 10^{-17} \mathrm{~cm}^{3} / \text { molecule } / \mathrm{sec} \\
& k_{2}=4.8 \times 10^{-15} \mathrm{~cm}^{3} / \text { molecule } / \mathrm{sec} \\
& k_{3}=2.62 \times 10^{-11} \mathrm{~cm}^{3} / \text { molecule } / \mathrm{sec} \\
& k_{4}=2.3 \times 10^{-12} \mathrm{~cm}^{3} / \text { molecule } \mathrm{sec}
\end{aligned}
$$

The individual reverse reaction rates were calculated from these rates using the appropriate equilibrium constant from the JANAF tables [47].

The equilibrium concentration of $N 0$ in the presence of $\mathrm{N}_{2}, \mathrm{O}_{2}, \mathrm{~N}$, and 0 at $2700^{\circ} \mathrm{K}$ and $20 \mathrm{~atm}$ total pressure (99 percent inert dilue, it) was calculated as 0.000385 , so that a vanishingly small amount of the original 0.01 concentration of NO will remain if the reactions are able to proceed to equilibrium. The equilibrium concentrations of the other species would be:

$$
\begin{aligned}
& {\left[\mathrm{N}_{2}\right]=0.0048} \\
& {\left[\mathrm{O}_{2}\right]=0.0046} \\
& {[0]=0.00054} \\
& {[\mathrm{~N}] \cong 2 \times 10^{-\mathrm{B}}}
\end{aligned}
$$

so that if the reactions have time to proceed to equilibrium under these conditions, virtually all of the original NO will be decomposed to $\mathrm{N}_{2}$ and $\mathrm{O}_{2}$. This fact alone is insufficient to disallow the use oi No as an absorber in this application, however, because NO is unstable even at room temperature, and it is the rate at which the equilibrium is attained that will determine whether NO will be sufficiently stable in the cyclic process of the heat engine.

A typical cycle time in a $7-\mathrm{kW}$ heat engine, whether it be steady flow or cyclic, is of the order of $10 \mathrm{msec}$. The time that the gas remains heated will be some fraction of this but not appreciably less. In order to be useful, the NO must not appreciably decompose in times of this order, particularly in a closed cycle system with a long planned lifetime, since there will be no regeneration of the No in the cooler portions of the cycle because of the chain reaction nature of the decomposition. In the presence of the closed chain reactions (b) and (c), steacy state concentrations of $N$ and 0 are rapidly attained so that 


$$
\frac{d[N]}{d t}=\frac{d[0]}{d t}=0
$$

The rapidity of attainment of this steady state witl be discussed further below. Under this assumption, the rate of decomposition of No may be written

$$
\begin{aligned}
\frac{d[N O]}{d t}= & -2 \times 1.4 \times 10^{-17}\left(\left[\mathrm{NO}^{2}-4.02 \times 10^{4}\left[\mathrm{~N}_{2} \mathrm{O}\right][0]\right)-\right. \\
& 2 \times 4.8 \times 10^{-15}\left([\mathrm{NO}][0]-1.8 \times 10^{3}[\mathrm{~N}]\left[\mathrm{O}_{2}\right]\right)
\end{aligned}
$$

Similar rate equations for 0 and $\mathrm{N}$ can be rearranged under Equation (9) to reduce this expression to

$$
\frac{\mathrm{d}[\mathrm{NO}]}{\mathrm{dt}}=-2.25 \times 10^{-17}[\mathrm{NO}]^{2}\left(1+4.8 \frac{[\mathrm{O}]}{[\mathrm{NO}]}\right)
$$

Since each removal of an 0 atom by reaction (d) also removes an $\mathrm{N}_{2} \mathrm{O}$ molecule, the 0 atom concentration will be equal to the $\mathrm{N}_{2} \mathrm{O}$ concentration in steady state. This, together with the rate equations represented by Equation (9), can be solved to relate the 0 atom concentration to the NO molecule concentration in steady state, which reduces Equation ( 11 ) to

$$
\frac{d[\mathrm{NO}]}{\mathrm{dt}}=-4.32 \times 10^{-17}[\mathrm{NO}]^{2}
$$

Integration of Equation (12) gives

$$
\frac{1}{[N O]_{0}}-\frac{1}{[N O]}=-4.32 \times 10^{-17} t
$$

In order to examine how rapidly the steady state is established, an exact integration of the five rate equations for the concentrations of NO, $0, \mathrm{~N}_{2} \mathrm{O}, \mathrm{N}, \mathrm{N}_{2}$, and $\mathrm{O}_{2}$ was carried out for the first two msec. It was found that the steady state was attained within the first $0.7 \mathrm{msec}$. Table 11, displaying the results of this full integration and the results of the steady state solution given by Equation (13), is shown below. 
Table 11

Temporal Decomposition of No

\begin{tabular}{ccc}
\hline \hline IME (mSec) & {$[\mathrm{NO}] /[\mathrm{NO}]_{0}(\mathrm{Eq} \cdot(13))$} & {$[\mathrm{NO}] /[\mathrm{NO}]_{0}$ (EXACT INTEG.) } \\
\hline 0.4 & 0.9907 & 0.989 \\
1.0 & 0.977 & 0.975 \\
2.0 & 0.955 & 0.954 \\
3.0 & 0.934 & \\
5.0 & 0.895 & \\
10.0 & 0.810 & \\
\hline
\end{tabular}

These results reveal that the system of reactions (a) through (d) is capable of appreciably reducing the initial concentration of NO in periods of time less than the cycle time of a typical heat engine. Even if the engine speed is increased and the heating phase is shortened, the amount of No lost during each cycle is unacceptably high for a closedcycle system with a long planned working life. For this reason, No is not a useful absorber gas for the present application, even though its absorption properties appear well suited.

The absorption transitions from the lower vibrational levels of CO were found to require more energetic photons than those provided by existing Co lasers. The energy level spacing is decreased when heavier isotopes are substituted for the carbon and oxygen. An investigation was made of resonances between the laser lines shown in Figure 32, and the absorption lines of $\mathrm{CO}$ isotopes. The wavelengths of $\mathrm{CO}$ isotope transitions were calculated using measured Dunham coefficients [48]. Several near coincidences on low vibrational levels of $\mathrm{C}(13) \mathrm{O}(18)$ were found. The absorption lines near several of the stronger laser lines are given in Table 12. Similar near coincidences occur with all of the laser 1 ines. It is apparent from the dai, presented in Table 12 that a supersonic laser is more ideally matched to a low temperature absorber. 
Table 12

${ }^{12} c^{16} 0$ Laser and ${ }^{13} c^{18} 0$ Absorber Lines

\begin{tabular}{|c|c|c|c|c|}
\hline \multicolumn{2}{|c|}{ LASER LINE $\left(\mathrm{cm}^{-1}\right)$} & P/P TOTAL & \multicolumn{2}{|c|}{ ABSORBER LINE $\left(\mathrm{cm}^{-i}\right)$} \\
\hline \multicolumn{5}{|c|}{ Subsonic Laser: } \\
\hline $7-6$ P16 & 1923.15 & 0.240 & $\begin{array}{ll}0-1 & P 31 \\
1-2 & \text { P25 } \\
4-5 & \text { P7 } \\
6-7 & \text { R6 } \\
7-8 & R 14 \\
7-8 & R 15 \\
8-9 & R 24 \\
9-10 & R 34 \\
9-10 & R 35\end{array}$ & $\begin{array}{r}1921.93 \\
1924.30 \\
1923.60 \\
1922.58 \\
1922.07 \\
1924.86 \\
1924.05 \\
1922.15 \\
1924.20\end{array}$ \\
\hline $7-6 \quad P 17$ & 1918.98 & 0.101 & $\begin{array}{ll}0-1 & P 32 \\
1-2 & P 26 \\
4-5 & P 8 \\
5-6 & P 1 \\
6-7 & R 5 \\
7-8 & R 13 \\
8-9 & R 22 \\
9-10 & R 33\end{array}$ & $\begin{array}{l}1917.56 \\
1920.12 \\
1920.02 \\
1920.67 \\
1919.48 \\
1919.26 \\
1979.09 \\
1920.06\end{array}$ \\
\hline
\end{tabular}

Supersonic Laser:

\begin{tabular}{|c|c|c|c|c|}
\hline 4-3 P10 & 2025.08 & 0.053 & $\begin{array}{ll}0-1 & \text { P5 } \\
1-2 & \text { RI } \\
2-3 & \text { R8 } \\
3-4 & \text { R16 } \\
4-5 & \text { R25 }\end{array}$ & $\begin{array}{l}2025.93 \\
2026.47 \\
2025.16 \\
2024.80 \\
2024.50\end{array}$ \\
\hline 4-3 PII & 2021.00 & 0.051 & $\begin{array}{ll}0-1 & \text { P6 } \\
2-3 & R 7 \\
3-4 & R 15 \\
4-5 & R 24 \\
5-6 & R 34\end{array}$ & $\begin{array}{l}2022.29 \\
2022.00 \\
2021.93 \\
2021.96 \\
2021.01\end{array}$ \\
\hline 5-4 P10 & 1999.15 & 0.050 & $\begin{array}{ll}0-1 & P 12 \\
l-2 & P 6 \\
2-3 & R 0 \\
3-4 & R 8 \\
4-5 & R 16 \\
5-6 & R 24 \\
5-6 & R 25 \\
6-7 & \text { R35 }\end{array}$ & $\begin{array}{l}1999.81 \\
1998.41 \\
1999.04 \\
2000.94 \\
2000.41 \\
1997.39 \\
1999.90 \\
1998.34\end{array}$ \\
\hline
\end{tabular}


The $C(13)$ and $0(18)$ isotope is not radioactive. A calculation was made of the stability of $\mathrm{CO}$ at high temperatures. The bond energy of $\mathrm{CO}$ is very high at $256.9 \mathrm{kcal} / \mathrm{mole}$. Equilibrium concentrations of carbon, oxygen, or carbon dioxide formed by the dissociation of $\mathrm{CO}$ at $3000^{\circ} \mathrm{K}$ and $20 \mathrm{~atm}$ are all jess than $10^{-5}$ percent. Therafore, the CO isotope will be stable at the highest cycle tetiera ires.

The absorption coefficients of each of the lines of the $\mathrm{CO}$ i st $s$ were calculated as a function of temperature, pressure, and mixtire composition. The absorption coefficient at any wave number, w. can be shown to be [49]

$$
\alpha_{\gamma}=\frac{1}{8 \pi} \frac{A_{u \ell}}{\omega^{2}} \frac{g_{u}}{g_{\ell}} N_{\ell}\left[1-\left(g_{\ell} / g_{u}\right)\left(N_{u} / N_{\ell}\right)\right] f(\omega)
$$

where the $g^{\prime} s$ are the degeneracies of the upper and lower levels, $f(w)$ is a line shape factor, and $A_{u l}$ is the Einstein coefficient for spontaneous emission. The Einstein coefficient may be related to the matrix element of the molecular dipole moment, which can be separated into a vibrational component and a rotational component [50],

$$
A_{u \ell}=\frac{64 \pi^{4}}{3 h} \frac{\omega^{3}}{g_{u}}\left|M_{u \ell}\right|^{2} s_{\ell} u
$$

where $\left|M_{u l}\right|^{2}$ is the square of the pure vibrational matrix element and $S_{\ell} u$ is the square of the pure rotational matrix element called the Honl London factor. For the vibrational transition within the co molecule [50],

$$
\begin{gathered}
S_{\ell} u=J_{\ell} \text { for a } P \text { transition } \\
S_{\ell} u=J_{\ell}+1 \text { for an } R \text { transition }
\end{gathered}
$$

where $J_{\ell}$ is the rotational quantum number of the lower state.

The ratio of upper to lower level number densities can be related to the wavenumber of the absorbed radiation, $\omega$, and the lower level number density can be related to the total number density divided by the vibrational and rotational partition function times the exponential of the ratio of the lower level energy divided by $k T$. 
For the pressure levels anticipated, the lines will be primarily collision broadened, giving a Lorentzian line shape function when Equation (14) is integrated over $w$. With these substitutions and integration over $w$, Equation (14) can be written in terms of the wavenumber of the center of the absorber line, $\omega_{a}$, and the wavenumber of the laser line, $\omega_{\ell}$,

$$
\alpha=\frac{4 \pi^{2} \omega_{d}}{3 h c}\left|M_{u \ell}\right|^{2} S_{u} \ell^{X} \frac{C_{0}\left(1-e^{-\theta_{v} / T}\right)}{k T^{2}} \theta_{r} e^{-E_{\ell} / k T} \frac{\left(1-e^{-h c \omega_{a} / k t}\right) \Delta \omega}{\left(\omega_{\ell}-\omega_{a}\right)^{2}+\left(\Delta \omega / 2 j^{2}\right.}
$$

where $X_{C O}$ is the mole fraction of $\mathrm{CO}$ and $\mathrm{p}$ is the total pressure. The energy difference between the ground level and the lower absorber level, $E_{\ell}$, was calculated using the Dunham coefficients for the $\mathrm{CO}$ isotope and the characteristic temperatures for rotation, $\theta_{r}$, and vibration, $\theta_{v}$, were derived from these calculations to be $2.75^{\circ} \mathrm{K}$ and $2946^{\circ} \mathrm{K}$, respectively.

The values for the square of the vibrational matrix element which depend upon the vibrational quantum number of the lower level were derived from the measurements of Varanasi [51] and those of Djeu and Sear]es [52]. The full-width, half-maximum linewidth was calculated using optical cross sections given by Penner [53],

$$
\Delta w=2.067 \times 10^{-5} \frac{\mathrm{P}}{\mathrm{T}}\left(\mathrm{x}_{\mathrm{CO}}+0.4824 \mathrm{X}_{\mathrm{Ar}}+0.3993 \mathrm{X}_{\mathrm{He}}\right)
$$

where $\Delta \omega$ is measured in inverse centimeters, the total pressure in Pascals, and the temperature in degrees Kelvin. The argon appearing in Equation (17) is used to characterize the high molecular weight component of the inert working gas in the cycle, and the helium represents the light component.

The rotational constant for $\mathrm{CO}$ shows that the individual $\mathrm{ab}-$ sorption lines are spaced 3.82 inverse $\mathrm{cm}$ apart. Equation (17) shows that for a mixture of $98 \mathrm{Ar} / 1 \mathrm{CO} / 1$ He at $300^{\circ} \mathrm{K}$, a pressure of $7.38 \times 10^{6}$ Pascals $(73 \mathrm{~atm})$ is needed to cause line overlap at $300^{\circ} \mathrm{K}$. This pressure is 1.83 times higher at $1000^{\circ} \mathrm{K}$. Since the pressures occuring in the cycle lie below these levels, the fully continuous absorption analys is of Caledonia [42] could not be used, and each absorber line had to be considered individually.

A computer code was generated that searched for all the absorption lines of the isotopic C0 within $\pm 2 \mathrm{~cm}^{-1}$ of the laser lines specified by either the subsonic laser spectrum or the supersonic 
laser spectrum shown in Figure 32. The absorption coefficient for each absorber line was then calculated using Equation(16), and the total absorption summed for each laser line. The amount of intensity absorbed in a distance $x$ can be written

$$
\Delta I=I_{0}\left(1-e^{-\alpha x}\right)
$$

The data of Figure 32 was utilized to relate the incident intensity on each line to the total intensity times a power fraction $F$. The characteristic length for absorption can be written in terms of the summed practical absorption coefficients as

$$
L=1 / \Sigma \alpha_{i} F_{i}
$$

where $i$ refers to the several laser lines included.

Once the laser energy is absorbed into the vibrational modes of the $\mathrm{CO}$ isotopes, it must be transferred to the translational modes as rapidly as possible in order to be used in the thermodynamic cycle. This process of relaxation of the vibrational energy must be characterized by the rate equation,

$$
d E / d t=\left(E^{\star}-E\right) / \tau
$$

Where $E^{*}$ is the equilibrium level of vibrational energy, and $\tau$ is a characteristic time for the deactivation process. For a mixture of gases, the characteristic deactivation time is

$$
\tau=\frac{k T}{p}\left(1-e^{-\theta} v^{/ T}\right)^{-1}\left(\Sigma x_{i} k_{i}\right)^{-1}
$$

where $p$ is the total pressure, $\theta_{v}$ the characteristic temperature of vibration, $x$ the constituent mole fraction, and $k$ the rate constant for that constituent deactivating $\mathrm{CO}$. Mixtures of argon and helium with the isotopic $C 0$ absorber were considered. The rate constants for these constituents were taken from the work of Millikan and White [54] and that of Miller and Millikan [55]. These rate constants are 


$$
\begin{array}{ll}
k_{C O}=2.994 \times 10^{-13} & \exp \left(-219.3 / \mathrm{T}^{1 / 3}\right) \mathrm{m}^{3} / \mathrm{sec} \\
k_{A r}=3.372 \times 10^{-12} & \exp \left(-270.9 / \mathrm{T}^{1 / 3}\right) \mathrm{m}^{3} / \mathrm{sec} \\
k_{H e}=5.012 \times 10^{-16} & \exp \left(-115.1 / \mathrm{T}^{1 / 3}\right) \mathrm{m}^{3} / \mathrm{sec}
\end{array}
$$

The helium rapidly deactivates the $\mathrm{CO}$ being 870 times faster than argon and 56 times faster than $\mathrm{CO}$ itself at $1000^{\circ} \mathrm{K}$.

The characteristic deactivation time must be short to avoid bleaclling of the absorber. Bleaching occurs when laser energy is absorbed faster than the upper level can be collisionally deactivated causing the upper leve] population density to approach the lower level population density. The absorption coefficient given in Equation (14) then diminishes substantially and the laser energy passes on through the medium. An approximate analysis relating the absorbed laser power density to the vibrational deactivation time in the presence of bleaching will be described. The molecule will be assumed to be a harmonic oscillator with absorption occurring between the $v$ and $v+1$ states. The equilibrium number density of these states is

$$
\begin{gathered}
N_{u}^{*}=g_{u} N_{t}\left(1-e^{-\theta^{\prime}}\right) \exp \left[-(v+1) \theta_{v} / T\right] \\
N_{l}^{*}=g N_{t}\left(1-e^{-\theta} v / T\right) \exp \left(-v \theta_{v} / T\right)
\end{gathered}
$$

It will be assumed that under bleaching conditions the laser energy is added faster than the vibrational levels of the absorber molecule can equilbrate with each other so that the sum of the upper and lawer level number densities will be constant equal to the sum of Equations (23) and (24). From Equation (14), the condition for vanishing absorption is

$$
g_{u} N_{\ell}=g_{\ell} N_{u}
$$

so that the upper level number density under bleaching conditions is

$$
N_{u}=g_{u} \frac{N t}{2}\left(1-e^{-\theta_{v} / T}\right)\left(e^{-Q_{v} / T}+g_{l} / g_{u}\right) \exp \left(-v \theta_{v} / T\right)
$$

The energy per unit volume needed to change this upper level density from that given in Equation (23) to that in Equation (26) is equal to the energy absorbed per transition, $h v=k \theta_{v}$, times the differences

$$
\frac{E}{v}=\frac{N_{t}}{2}\left(1-e^{-\theta_{v} / T}\right)\left(g_{\ell}-g_{v} e^{-\theta_{v} / T}\right) k \theta_{v} \exp \left(-v \theta_{v} / T\right)
$$


This energy must be absorbed in a time comparable with vibrational deactivation time for bleaching to occur. Therefore, the bleaching power per unit volume is

$$
\frac{P_{B}}{V}=\frac{x p}{2 \tau} \frac{\theta_{v}}{T} g_{l}\left(1-e^{-\theta_{V} / T}\right)\left(1-\frac{g_{u}}{g_{\ell}} e^{-\theta_{v} / T}\right) \exp \left(-v \theta_{v} / T\right)
$$

where $X$ is the mole fraction of the absorber, $p$ is the total pressure, and $v$ is the vibrational quantun number of the lower level.

Calculations of the power density to bleach the isotopic $\mathrm{CO}$ absorber were made for a mixture of $98 \mathrm{Ar} / 1 \mathrm{CO} / 1 \mathrm{He}$ under the most critical conditions occurring in the 0tto cycle: $p=1.89 \times 10^{6}$ Pascals $(18.7$ atm), $T=881^{\circ} \mathrm{K}$. Conditions occurring in the Brayton cycle are less favorable for bleaching. The characteristic time for vibrational deactivation is $2.03 \times 10^{-4} \mathrm{sec}$. The power density necessary to bleach the $0-1$ transition is $197.2 \mathrm{~W} / \mathrm{cm}^{3}$. Since the absorbing volume will be approximately $1000 \mathrm{~cm}^{3}$, the laser power necessary to saturate this transition is $147 \mathrm{~kW}$. The $98 \mathrm{Ar} / 1 \mathrm{CO} / 1 \mathrm{He}$ mixture is therefore limited to applications of less than $70 \mathrm{~kW}$ electrical output when an overall conversion efficiency of 50 percent is assumed. Larger engines powered by $C 0$ lasers can be built using a larger concentration of helium in the absorbing/working gas, since the characteristic time for collisional deactivation decreases rapidly as helium is substituted for argon in the mixture. At $881^{\circ} \mathrm{K}$, the characteristic time for collisional deactivation is inversely proportional to the sum

$$
\mathrm{X}_{\mathrm{Ar}}+19.32 \mathrm{x}_{\mathrm{CO}}+1700 \mathrm{X}_{\mathrm{He}}
$$

so that doubling the helium fraction to 2 percent at the expense of the argon will approximately halve the characteristic time for vibrational relaxation and double the laser power required to bleach the absorber.

The laser power deposited in the absorbing gas must be collisionally transferred to the translational mode before it is re-emitted by the spontaneous radiative decay of the molecule. This can be assured if the characteristic time for collisional deactivation is much shorter than the radiative lifetime of the molecule. The radiative lifetime is defined as the inverse of the Einstein coefficient for spontaneous emission given in Equation (15). The value for the 1-0 transition has been

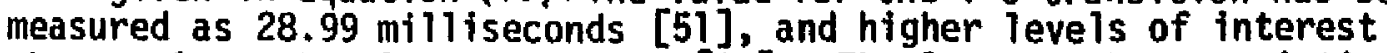
show no less than $1 / 5$ this value [52]. The longest characteristic collisional deactivation time of the $98 \mathrm{Ar} / 1 \mathrm{He} / \mathrm{iCO}$ mixture occurring in either the 0tto or Brayton cycle is 0.2 milliseconds, so that appreciab le spontaneous emission should not occur before thermalization of the absorbed energy takes place. 
Absorption calculations were made for a $98 \mathrm{Ar} / 1 \mathrm{CO} / 1 \mathrm{He}$ mixture under four sets of thermodynamic conditions and for both the subsonic and supersonic laser. The sets of thermodynamic conditions correspond to the end points of the heating phase of the 0tto cycle and the Brayton cycle analyzed in this study. The conditions are given in Table 13. The absorbed power was calculated using Equation (15) for 22 subsonic laser lines and 48 supersonic laser lines, the strongest of which are given in Table 12. The results of the absorption calculations are presented in Table 14 as the surmation term given in Equation (19) for each of the four cases considered. The $\mathrm{CO}$ fraction can be increased to about 10 percent with 11 ttle change in thermodynamic quantities, providing an increase in absorption coefficient by an order of magnitude.

Table 13

Themodynamic State of $98 \mathrm{Ar} / 1 \mathrm{CO} / 1 \mathrm{He}$ Gas

Otto Cycle:

1. $1.89 \times 10^{6}$ Pascals (18.7 atmospheres), $881^{\circ} \mathrm{K}$

2. $6.438 \times 10^{6}$ Pascals (63.7 atmospheres), $3000{ }^{\circ} \mathrm{K}$

Brayton Cycle:

3. $1.86 \times 10^{6}$ Pascals (18.4 atmospheres), $1098^{\circ} \mathrm{K}$

4. $1.86 \times 10^{6}$ Pascals (18.4 atmospheres), $3000^{\circ} \mathrm{K}$

Table 14

Sunmed Absorption Coefficient CYCLE/LASER SUBSONIC SUPERSONIC

1. Otto Cycle Low T (18.7 atm,881 $\left.{ }^{\circ} \mathrm{K}\right)$

$0.01835 / \mathrm{cm}$

$0.1509 / \mathrm{cm}$

2. Otto Cycle High $T\left(63.7 \mathrm{~atm}, 3000^{\circ} \mathrm{K}\right)$

$0.06384 / \mathrm{cm}$

$0.07300 / \mathrm{cm}$

3. Brayton Cycle Low T (18.4 atm, $1098^{\circ} \mathrm{K}$ )

$0.02185 / \mathrm{cm}$

$0.08717 / \mathrm{cm}$

4. Brayton Cycle High T (18.4 atm, $\left.3000^{\circ} \mathrm{K}\right)$

$0.01719 / \mathrm{cm}$

$0.02407 / \mathrm{cm}$ 
The results themselves will first be discussed and then their implications on engine design will be considered. The otto cycle represents a constant density heating process so that the same number of absorbing particles are available throughout the heating phase between Conditions 1 and 2 of Table 13. As the translational temperature of the absorber rises, the population of the upper levels increases and more absorption of emission from the high levels characteristics of the subsonic laser will occur. In addition, the pressure level increases between Conditions 1 and 2 of the 0tto cycle broadening the absorption lines and increasing the absorption of slightly displaced laser lines. The total absorption is increased by a factor of 3.5 beteen Conditions 1 and 2 as a result of these effects. The emission lines of the supersonic laser extend to lower vibrational levels and are more well matched to the absorber lines at the low temperature represented by Condition 1 giving a higher absorption than that of the subsonic laser. As the absorber temperature rises, however, more of the absorber molecules move out of these lower level states and into high level states decreasing absorption of the low lying supersonic co laser lines. Even with the beneficial pressure broadening effect this causes a decrease of a factor of 2 in the absorption of supersonic $\mathrm{CO}$ laser radiation between Conditions 1 and 2 of the 0tto cycle. These results show that an optimum laser for supplying energy to this 0tto cycle would provide emission in the region between the spectra of the two lasers considered in this study. These spectrum calculations show that by selective tuning techniques, the emission of a $\mathrm{CO}$ laser and the choice of absorbing medium can probably be tailored to provide the desired absorption throughout the heating phase of the otto cycle.

The results presented in Table 14 demonstrate the manner in which these calculations affect the design of a co laser heated 0tto cycle. If the subsonic co laser characterized by the emission spectrum shown in Figure 32 were used to power the 0tto cycle characterized by the data given in Table 13, the critical absorption condition wil1 occur at the beginning of the heating phase (Condition 1). Equation (19) applied to Condition 1 shows the characteristic length for absorption is $55 \mathrm{~cm}$ in this case. An absorption path of at least twice this length is required to couple the laser energy adequately. This path length may be obtained by reflecting the laser energy several times from the walls of the absorber volume; however, minimum absorption conditions for the 0tto cycle given in Table 14 correspond to the minimum volume portion of the cycle. To ensure total absorption of the energy delivered from a subsonic co laser without excessive wall reflections and attendant wall absorption, the concentration of $\mathrm{CO}$ isotope must be raised in the gas. The absorption coefficient given in Equation (16) varies approximately linearly with $c 0$ isotope fraction. To afford adequate absorption at the beginning of heating phase of the 0tto cycle when the distance between the input window and reflecting piston crown is $2 \mathrm{~cm}$, the $\mathrm{CO}$ isotope fraction must be raised to 10 percent at the expense of the argon in the mixture. The ratio of specific heats 
will then be altered from 1.662 for the $98 \mathrm{Ar} / 1 \mathrm{CO} / 1 \mathrm{He}$ mixture to 1.625 for this $89 \mathrm{Ar} / 10 \mathrm{CO} / \mathrm{lHe}$ mixture. This lowers the ideal efficiency of the otto cycle

$$
n=1-r^{l-k}
$$

from 0.826 to 0.808 for a compression ratio $r$ of 14 . This two percent loss in ideal cycle efficiency must be balanced aga inst the increased system cost represented by the additional co isotope required for an ot to cycle powered by a subsonic co laser. The characteristic time for deactivation of the $89 \mathrm{Ar} / 10 \mathrm{CO} / 1 \mathrm{He}$ mixture is less than that of the original 98Ar/1CO/THe mixture according to Equation (29) so that the bleaching and spontaneous emission losses would not be important.

If the supersonic CO laser that is characterized by the emission spectrum given in Figure 32 is applied to the otto cycle characterized by the data given in Table 13, the critical absorption phase occurs at the high temperature Condition 2 where Equation (29) gives $L=13.7 \mathrm{~cm}$ for the $98 \mathrm{Ar} / \mathrm{IHe} / 1 \mathrm{CO}$ mixture. The pathlength required to couple the laser energy is thus about $27 \mathrm{~cm}$. If the device has a distance of $2 \mathrm{~cm}$ between the input window and refiecting piston crown, the co isotope fraction would have to be raised to 3 percent to adequately couple the laser energy into the working gas with only three reflections. The resulting $96 \mathrm{Ar} / 3 \mathrm{CO} / 1 \mathrm{He}$ mixture will provide essentially the same cycle efficiency. Through the use of a $c 0$ laser which provides lower vibrational lines (Fig. 32), the required $\mathrm{CO}$ isotope concentration has been reduced by a factor of 5 for the 0tto cycle. Examination of Table 13 shows that an intermediate laser with emission spectrum tailored to the otto cycle heating phase could reduce the required $\mathrm{CO}$ concentration even further by keeping the summed fractional absorption coefficient relatively constant between Conditions 1 and 2 but higher than $0.07 / \mathrm{cm}$.

In the case of the Brayton cycle, the data given in Table 14 show that the number of particles is not held constant and as the mixture is heated at constant pressure the density together with the number of absorbers in a fixed volume decrease by a factor of 2.7 between Condition 3 and Condition 4 . When the supersonic $C 0$ laser is used to supply power to the Brayton cycle the summed fractional absorption decreases by a factor of 3.6 between Conditions 3 and 4 . The additional decrease beyond the decreased density effect can be traced to the vibrational population shift to higher levels in the absorber as the temperature is raised. This effect was discussed above for the 0tto cycle. It causes the primary absorbing line to move away from the strong laser emission lines thereby decreasing the fraction of laser energy absorbed. This effect is reversed when the subsonic laser is used to power the Brayton cycle. In this case the increase in upper level populations in the absorber as the temperature is raised strengthen the absorption of 
the high level laser lines emitted by the subsonic laser. This effect also appeared in the Otto cycle described above. This enhancement of absorption is not sufficient to offset the decrease in density, however, and the summed fractional absorption of subsonic Co laser power drops by 20 percent between Conditions 3 and 4 of the Brayton cycle.

When the subsonic laser is applied to the Brayton cycle conditions the characteristic absorption length will be $58 \mathrm{~cm}$ at the critical high temperature Condition 4 . In order to couple the laser energy, a path length of $116 \mathrm{~cm}$ is required, which is not unrealistic for a folded beam within the heat addition region of the Brayton engine. To provide adequate absorption, however, the $C O$ isotope fraction should be raised to approximately 4 percent at the expense of the argon, giving a $95 \mathrm{Ar} / 4 \mathrm{CO} / 1 \mathrm{He}$ mixture. With one reflection, the length of the heater required is then approximately $15 \mathrm{~cm}$ and the small effect on the ratio of specific heats witl negligibly affect the cycle efficiency.

The supersonic laser applied to the Brayton cycle at the critical Condition 4 gives $L=41 \mathrm{~cm}$, showing that a $82-\mathrm{cm}$ absorption path is required. This requirement can be reduced to $14 \mathrm{~cm}$ by raising the $c 0$ isotope fraction to 3 percent at the expense of the argon in the working gas mixture. Again it is seen that the supersonic $c 0$ laser is better suited to the task of providing power to the cycle; however, in the case of the Brayton cycle the differences are less than in the case of the otto cycle. It should be kept in mind that is is possible to tailor the operation of the CO laser so that the shift of absorption is minimized as the temperature is increased and the overali absorption level is not too low.

This portion of the study has shown that a co laser coupled with a $c 0$ isotope as an absorber can be utilized to power a laser heat engine. The supersonic $C 0$ laser emission spectrum [44] was superior to the subsonic 60 laser emission spectrum [43] in this analysis; however, the results indicate that a co laser should be tailored to better match a specified engine cycle. Such a tailored co laser can be realized through the adjustment of such operational parameters as gas mixture, gas temperature, energy loading and through the use of selective mirror coatings or intra-cavity gratings.

\subsubsection{Absorption of Radiation from $\mathrm{CO} 2$ Lasers}

The $\mathrm{CO}_{2}$ laser produces infrared radiation on one of several lines, the strongest being at 10.6 microns. Absorbing molecules have a characteristic vibrational temperature of about $1380^{\circ} \mathrm{K}$. This temperature 
is not as attractive as the higher temperatures accessible through the use of $\mathrm{CO}$ lasers. Yet $\mathrm{CO}_{2}$ laser technology at high power, both $C W$ and pulsed, is available now for performing interesting experiments. With careful attention to loss reduction, laser heat engines can possibly be constructed in the near-term which operate efficiently at temperatures of $1380^{\circ} \mathrm{K}$.

In contrast to absorbers of $\mathrm{CO}$ laser radiation, the absorbers of $\mathrm{CO}_{2}$ laser radiation are more loosely bound polyatomic molecues such as $\mathrm{SF}_{6}$ and water which have wide absorption bands bracketing the $\mathrm{CO}_{2}$ laser radiation line. Since all of the $\mathrm{C}_{2}$ laser radiation can be carried by a single line, it is a relatively simple matter to match the absorber to the laser output.

Both $\mathrm{SF}_{6}$ and $\mathrm{D}_{2} \mathrm{O}$ exhibit good absorption of $\mathrm{CO}_{2}$ laser radiation. However, at elevated temperatures, $\mathrm{SF}_{6}$ undergoes an irreversible chemical deterioration leading to the precipitation of sulfur and the formation of $F_{2}$ and smaller polyatomic sulfur-fluorine compounds. Thus, we have concentrated on $\mathrm{D}_{2} \mathrm{O}$ which has $v_{2}$ band with center at $1178 \mathrm{~cm}^{-1}$ and width large enough to trap 9.26 -micron radiation. This corresponds to the $\mathrm{R}(22) \mathrm{CO}_{2}$ laser line, which can be easily excited.

The band structure of $\mathrm{D}_{2} \mathrm{O}$ was assumed to be similar to $\mathrm{H}_{2} \mathrm{O}$ so that $\mathrm{H}_{2} \mathrm{O}$ band absorption data, appropriately shifted in frequency, can be used. Specifically, the $v_{2}$ band center for $\mathrm{H}_{2} \mathrm{O}$ is $1595 \mathrm{~cm}^{1}$ [56]. The absorbing $0_{20}$ transition was found to be at 1079.86 $\mathrm{cm}^{-1}$ [57]. Thus, the point of the $\mathrm{H}_{2} \mathrm{O} v_{2}$ band similar to 1079.86 on the $D_{2} 0$ band would be located at

$$
\bar{v}_{\mathrm{H}_{2} \mathrm{O}}=\frac{1595}{1178} \bar{v}_{\mathrm{D}_{2} \mathrm{O}}=1462 \mathrm{~cm}^{-1}
$$

the absorption coefficient used for the $\mathrm{D}_{2} 09.26$-micron calculation is

$$
k(9.26 \mu)=0.63 / \mathrm{cm} / \mathrm{atm}
$$

taken from $\mathrm{H}_{2} \mathrm{O}$ absorption data at $1462 \mathrm{~cm}^{-1}$ [58].

Because the absorption for deuterated molecules has been generally found to be one-half of the absorption coefficient for the same band when hydrogen is present (e.g. $\mathrm{C}_{2} \mathrm{D}_{2}$ vs. $\mathrm{C}_{2} \mathrm{H}_{2}, \mathrm{C}_{2} \mathrm{D}_{4}$ vs. $\mathrm{C}_{2} \mathrm{H}_{4}[59]$, we take

$$
k_{D_{2} 0}(9.26)=1 / 2 k(9.26)
$$

for the $\mathrm{D}_{2} \mathrm{O}$ absorption coefficient. The product of gas pressure and absorption length is given by 


$$
e^{-k_{D_{2} 0^{P l}}}=0.05
$$

where 95 percent absorption has been assumed. Thus,

$$
P l=-\frac{\ln (0.05)}{0.5 k}=2 \times 4.76=9.52 \mathrm{~atm}-\mathrm{cm}
$$

Thus, 1 atm of $D_{2} 0$ requires an absorption length of $9.52 \mathrm{~cm}$ for 95 percent absorption.

For the 0tto cycle where laser absorption begins at a total gas pressure of approximately $20 \mathrm{~atm}, 1$ atm of $\mathrm{D}_{2} \mathrm{O}$ comprises a 5 percent mole fraction in the working gas mixture. The same pressure conditions apply to the Brayton cycles considered, as shown in Table 13. case is [5]

The power density required of the laser in the 0tto cycle

$$
P_{\text {0tto }}=\frac{3}{2} \frac{N k \Delta T}{t_{2}}=\frac{4.28}{t_{2}} \mathrm{~W} / \mathrm{cm}^{3}
$$

where $N$ is the working gas number density at 20 atm and $614^{\circ} \mathrm{K}$, $t_{2}$ is the laser pulse length, and $\Delta T=1400^{\circ} \mathrm{K}-614^{\circ} \mathrm{K}=786^{\circ} \mathrm{K}$ is the temperature rise during laser heating for a compression ratio of 6 . If the absorbing volume is a cylinder 1 absorption length deep and $3 \mathrm{~cm}$ in diameter, the Taser must supply 284 joules/ pulse, which can be readily supplied by existing lasers.

The power needed to bleach the absorber can be calculated from Equation (28):

$$
P_{B}=\frac{0.501}{\tau} \text { watts }
$$

Therefore, in order to avoid bleaching, the laser pulse length must be at least 568 times the vibrational relaxation time, which is about $10^{-7} \mathrm{sec}$, or $5.68 \times 10^{-5} \mathrm{sec}$. This represents a bulse length easily within the natural pulse length for existing large $\mathrm{CO}_{2}$ lasers, but does present some laser problems in the smaller $(\sim-10 \mathrm{~kW})$ sizes.

In contrast to 5- $\mu$ absorbers, the 9.26- $\mu$ absorbers are also good radiators at the peak cycle temperatures in question. For the case of $\mathrm{D}_{2} \mathrm{O}$, the radiation emitted at $1500^{\circ} \mathrm{K}$, for the conditions 
used in the 0tto cycle calculation, can be obtained from $\mathrm{H}_{2} \mathrm{O}$ band data. Data is available in discrete wave number interva]s [58], and the emission is given by

$$
W=2 \pi c^{2} h \Delta \nu \sum\left[1-\exp \left(\frac{k\left(v_{i}\right) \operatorname{lnc}}{k(10.6)}\right)\right] \quad \bar{v}_{i}^{3}\left[\exp \left(\frac{h c \bar{v}_{i}}{k t}\right)-1\right]^{-1}
$$

where $\Delta v$ is the width of each interval, $\bar{v}_{j}$ is the wave number at the center of the ith interval, and $c$ is the fractional amount of radiation transmitted. Detailed balance is used to relate the emission coefficient to the absorption coefficient. Also, since the absorption coefficients enter this expression onty in the ratio $k\left(\bar{v}_{j}\right) / k(9.26 \mu)$, the 0.5 factor associated with deuterated species cancels, and at $1500^{\circ} \mathrm{K}$,

$$
W=7.77 \text { watts } / \mathrm{cm}^{2}
$$

The total power radiated from a cylinder $1.5 \mathrm{~cm}$ in radius and 9.52 cm (absorption length) long is

$$
P=W \cdot A=523 \text { watts }
$$

The effect of the radiation loss is not as severe as the above might suggest. Suppose that the laser power input to an 0tto cycle averages 2 kilowatts (i.e., at 50 percent efficiency, the output would be 1 kjlowatt). Furthel", assume that the engine speed is $1200 \mathrm{rpm}$. Then, the energy input per cycle is

$$
E=\left(2 \times 10^{3}\right) /\left(\frac{1200}{60} \mathrm{rpm}\right)=100 \mathrm{~J}
$$

In one-half of the cycle time, when the gas is hot, the energy lost by radiation is

$$
E=523 \mathrm{~W} \frac{0.5}{20} \mathrm{sec}=12.5 \mathrm{~J}
$$

Therefore, the energy lost is 12.5 percent of the input energy. The available work lost is only about one-quarter of that value, 
or about 3 percent. Thus, if the cycle thermal afficlency wre 60 percent before including radiation losses. then to would be 57 percent after including radiation losses. The losses are probobly even smaller, since the peak temperature was used to ovaluate the emissivity. We conclude that $D_{2} 0$ has very good potential as the absorber in a near-term experiment uttlizing a $\mathrm{CO}_{2}$ leser comsy source.

\subsection{Mechanical-Electrical Conversion}

Conversion of mechanical energy, Initially frem the high temperature working flutd, to electricity can be accoplisho 1 i. rectly through a MHD or MPD genervetor or Indirectly through incer. mediate mechanical stages, such as turbin and then rotating generator. Each of these al tematives has wighe, volum. rollobil= ity, and malntenance charactertstics which must be coluted ond in: tegrated with the particular engine types studied. The principal

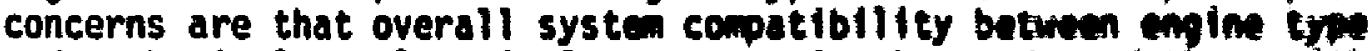
and mechanical-to-electrical converter is observed, and the mither of these two elements of the laser heat englne for outwoto the other. so that an approxiante optimu in terms of a linted set of criterta is established before the selection of bets ongline is made.

A prelininary basis for rating different engin concepts cen* ststs of rough system conftguratlons for a fixed pown outeut. siy one kilowatt. Questlons which imst be antwered concern the type of generators to be used, generator coolin, seils, lubricteten. wi. tage and frequency control. the production of of versus is eaver. two- versus three-phase power, and 80 forth, under the optimin cen. ditions of effictency, wight, voluen, and rollabllity.

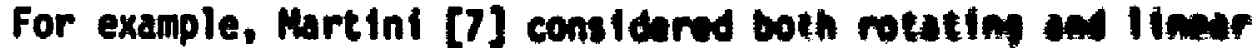
generators in his study and found a range of operating officiemetes, It is well known that high rotatlongl spends ylele hlower fflelewsy

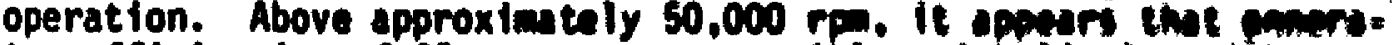
tor efficiencies of 95 porcent are possible, with litely whts on

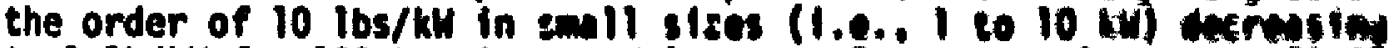

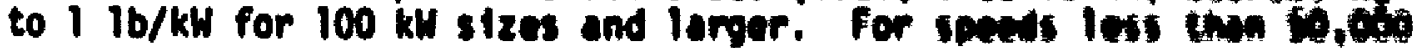
rpm, the generator efficlenctes decrease to 90 purcent or Itume ot

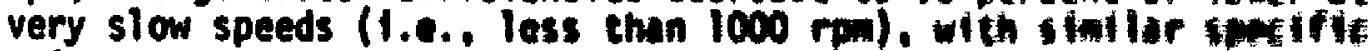
welghts.

Linear generators with high efflelency (90 to powen)

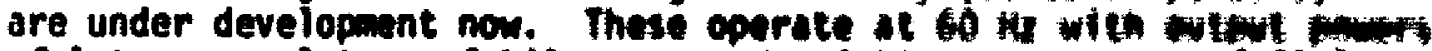
of 1 to several tens of kflowets and wight en the encer of

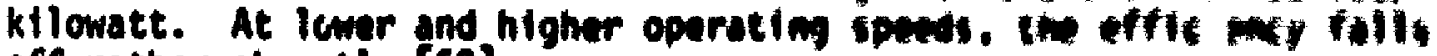
off rather abruptly [60]. 
The relative advantages of these two types of generators are the low weight and high speed efficiency of rotating generators, and the zaro toily mosion and $60 \mathrm{~Hz}$ efficiency of the jinear generator. Rotitilg generators are ideally suited to turbine heat engines, but require the conversion of piston linear motion to rotary motion, with resultant tilting torques on the piston as it rides up and down in tile cylinder. These torques will place special constraints on gas lubricated pistons where there are rather small restoring forces a a condition of a low leakage design. In this respect, linear geveito.'s appear superior because of their relatively low lateral motion There are torques even in a linear generator system which are caused by the electronagnetic interaction between the linear alternator and the field coils, but these can be kept quite small. Against these advantages, one must consider the higher specific weight of linear generators: nearly six times as large as rotating generators.

In most applications, $60 \mathrm{~Hz}$ operation is the most desirable, and both of the preceding types of generators can supply power in this form. Alternating current also has the advantage of simple voltage transformation. For this reason, the direct current power supplied by an MHD generator would be more cumbersome to use. Power conditioning would be required for many applications, adding to the total system wejght and decreasing the power output efficiency.

Generator cooling is required in order to maintain the insulation and lubricants in good working order. Coil cooling is particularly necessary to keep the conductor resistance low and to reduce joule heat losses. Liquid coolants are the most effective, and in sone designs, heat pipes may be the best way to transport that waste heat to the radiator. This waste heat may be as much as 10 to 20 percent of the total waste heat rejected by the system, so that some effort must be given to careful design for disposing of it. It would probably be worthwile designing a separate smali waste heat radiator optimized for rejecting that heat.

\subsection{Heat Rejection}

Heat must be rejected to space from the low temperature end of the therma: cycle, from waste heat lost at other points throughout the engine, and from engine parts which must be deliberately cooled. This can be accomplished indefinitely by radiation and, for short periods of time, by ejecting mass (e.g., spent working fluid) from the engine. We distinguish between direct heat rejection by radiation, where the principal wasie heat cones from the low temperature part of the cycle, and indirect heat rejection, where a coolant must be circulated as an intermediate step to conduct heat away from a higher temperature engine part and then the 
heat is rejected to outer space. Indirect heat rejection therefore involves a coolant and some type of internal heat exchanger (e.g., piston wall cooling jacket). Both types of heat rejection can occur in a single engine concept.

\subsubsection{Specific Radiator Area}

One of the major tradeoffs in heat rejection is between the size of the radiator, which varies inversely with the fourth power of the temperature at which heat is rejected, and cycle efficiency, which increases with decreasing heat rejection temperature. For example, heat rejection from a Brayton cycle occurs at constant pressure between temperatures $T_{4}$ (turbine exit) and $T_{7}$ (compressor inlet). The expression for specific radiator area is

$$
\frac{A}{p}=[(1-n) / n] \frac{1}{\operatorname{coT}_{a v}^{4}} \text { in } m^{2} / k W
$$

where $c$ is the enissivity of the radiator, $n$ is the thermal cycle effictency, and

$$
T_{a v}^{4}=\frac{3 T_{1}^{3} T_{4}^{3}}{T_{1}^{2}+T_{1} T_{4}+T_{4}^{2}}
$$

Is an approprlate average temperature for the radiation loss procoss. Mackay [61] shows that the efficiency

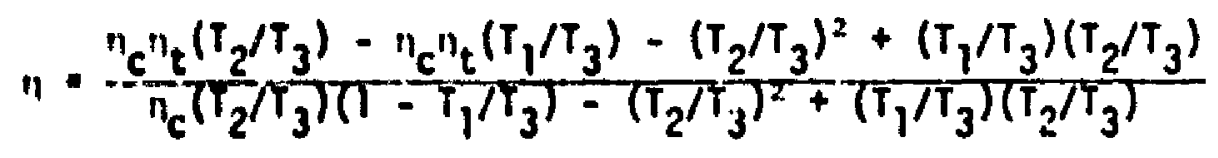

where $T_{2}$ is the compressor exit temperature and $T_{3}$ is the turbine Inlet tomperature, reaches a maximum when

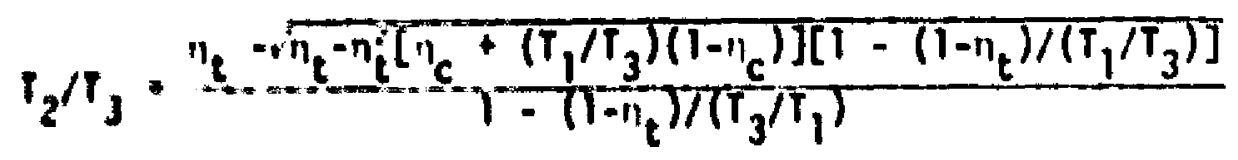

where the lowust temperature in the cycle. $T_{1}$, has been held contint. For maximm $n$. the specific radiator area is a mintmum. 
To compute the specific radiator area for a given ratio $T_{1} / T_{3}$, we must also use the relation

$$
T_{4}=T_{3}\left[1-n_{t}\left(1-\frac{T_{1} / T_{3}}{T_{2} / T_{3}}\right)\right]
$$

An example is shown in Figure 33, adapted from Mackay [61], to illustrate the sensitivity of specific radiator area on the compressor efficiency, $n_{c}$, and the turbine efficiency, $n_{t}$, for the given peak temperature, $1100^{\circ} \mathrm{K}\left(2000^{\circ} \mathrm{R}\right)$.

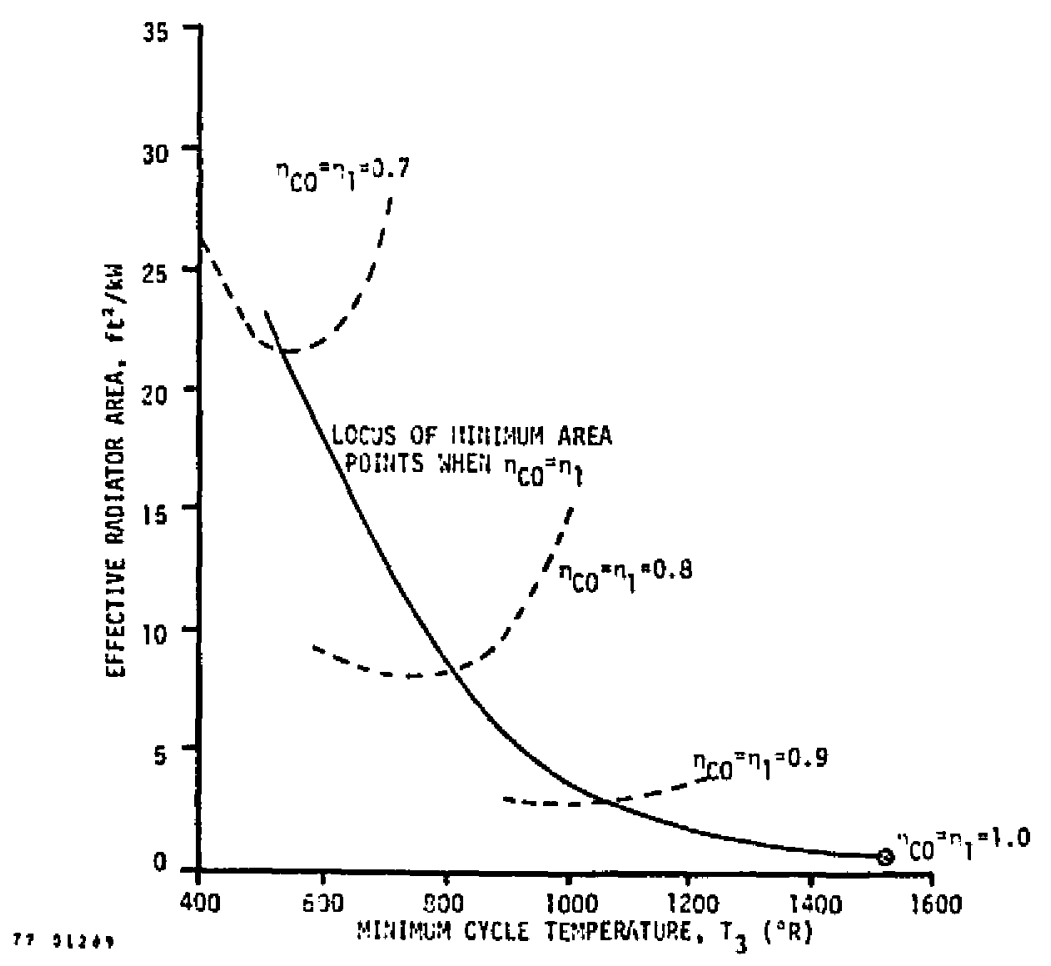

Figure 33. Minimum Radiator Area, Brayton Cycle (assumed condition: surface emissivities $=1.0$ ) 
A similar expression can be derived for an otto cycle, where the waste heat must be radiated from a constant volume. Again,

$$
\frac{A}{p}=\frac{1-n}{n} \frac{1}{E \sigma T_{a v}^{4}}
$$

In this case, we assume that the radiator volume is much larger than the piston volume, so that the addition of a fresh charge of hot exhaust gas will not perturb the mean radiator temperature, $T_{R}$, i.e. $T_{a V}=T_{R}$ is the maximum cycle temperature. This assumption also guarantees sufficient residence time in the radiator for each fresh charge of exhaust gas to cool off. Hence, we can minimize $A / P$ with respect to $n$ for a fixed minimum cycle temperature and maximum cycle temperature. A moment's consideration shows that $n_{\text {max }}$ is achieved for that critical compression ratio where all of thex gas heating from $\left(T_{\min }\right)_{\text {cycle }}$ to $\left(T_{\max }\right)_{\text {cyc }}$ is achieved by compression alone, and the work per cycle tends to zero. This rather unsatisfactory limit can be replaced by the maximum work 0tto cycle given by

$$
n=1-\sqrt{T_{\min } T T_{\max }}
$$

Then,

$$
(A / P)_{\max . \text { work cycle }}=\frac{\sqrt{T_{\min } / T_{\max }}}{1-\sqrt{T_{\min } / T_{\max }}} \frac{1}{\varepsilon \sigma T_{\min }^{4}}
$$

\subsubsection{Material Limits}

The radiator weight is generally proportional to the radiator area so that the scaling with power can be roughly approximated by Equations (31) to (34) for the Brayton cycle, or Equation (35) for the otto cycle. Perhaps the two most important considerations in determining the weight per unit area of the radiator are meteorite protection requiring thick tubes [62], and heat conduction requiring thin tubes [61]. The heat conduction aspect of radiator weight is compounded by the film resistance between the working fluid (or coolant) and the interior tube walls of the radiator. In designing Brayton power units, there is a competition between high molecular weights for efficiency in the turbine and compressor, and 
low molecular weights for good heat conduction in the radiator [20]. Sufficient to say that a detailed system-wide optimization of a space power plant must take all of these considerations into account. Here we simply summarize the likely range of radiator weights and materials.

Deidrich and Lieblein have given a comprehensive survey of materials problems associated with the design of radiators for space power plants [63]. Table 15 provides essential information on a selection of useful radiator materials for both Brayton and Rankine cycles, the latter often requiring corrosion-resistant materials. The relative radiator weights are shown in Figure 34, taken from Reference [63], where the weights are normalized to a beryllium radiator and where the power cycles are potassium and mercury Rankine cycles. Similar relative weights are to be expected from Brayton and other cycles, wi th the exception that the location of the weight minima may shift because the heat rejection process is no longer isothermal. Also, the absolute weight for a Brayton cycle radiator will be greater than for a Rankine cycle because the film resistance for a gaseous working fluid is high compared to a liquid (condensed) working fluid. Typical weights per unit area are 3 to $5 \mathrm{kG} / \mathrm{m}^{2}$ for tapered tube and fin radiators [64].

Table 15

Material Properties [63]

\begin{tabular}{|c|c|c|c|c|c|c|c|c|}
\hline \multirow{3}{*}{ MATERIAL } & \multirow{3}{*}{$\begin{array}{l}\text { DENSITY } \\
\text { lb/ft }\end{array}$} & $\begin{array}{c}\text { COHDUCT I OH } \\
\text { PARA:ETER } \\
K /, 1\end{array}$ & 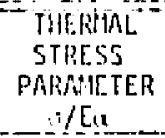 & $\begin{array}{c}\text { THFinAi } \\
\text { SHLCK } \\
\text { PARALETER } \\
\text { RK/E }\end{array}$ & $\begin{array}{l}\text { PENTTAAIOAT } \\
\text { RESISTAICE } \\
\text { PARAMETER } \\
\text { OWLE }\end{array}$ & \multicolumn{2}{|c|}{$\begin{array}{c}\text { SUEL IMATION } \\
\text { RATE } \\
\text { in./yEar }\end{array}$} & 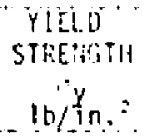 \\
\hline & & \multicolumn{7}{|c|}{ TEMPERATURE, ${ }^{\circ} \mathrm{K}$} \\
\hline & & 1030 & 920 & 920 & 1030 & 1030 & 1140 & 10.30 \\
\hline $\begin{array}{c}\text { cuilinerc inil } \\
\text { graph ite }\end{array}$ & 109 & 0.365 & 330 & $13.5 \times 10^{3}$ & $4.7 \times 10^{-3}$ & $<10^{-10}$ & $<10^{-2}$ & $2.4 \times 117^{4}$ \\
\hline beryll i un & 116 & 0.400 & 15 & 0.79 & 1.9 & $4.1 \times 10^{-1}$ & 0.15 & 2.1 \\
\hline $\begin{array}{c}\text { pyrolytic } \\
\text { graphite }\end{array}$ & 130 & $\left\{\begin{array}{l}0.590 \\
1.100\end{array}\right\}$ & 220 & 17.5 & 3.5 & $<10^{-10}$ & $<10^{-9}$ & 14.8 \\
\hline $\begin{array}{l}\text { wrought } \\
\text { a luritinum }\end{array}$ & 172 & $\cdots$ & --- & -- & $\cdots$ & $8 \times 10^{-3}$ & 0.3 & $\cdots$ \\
\hline $\begin{array}{l}\text { a luminum } \\
\text { powder } \\
\text { metalibrgy }\end{array}$ & 172 & $\cdots$ &.- & -- & --- & $8 \times 10^{-3}$ & 0.3 & $\cdots$ \\
\hline titanitull & 2133 & 0.039 & $21 *$ & $1.8^{\circ}$ & $\cdots$ & $<10^{-5}$ & $\cdot 10^{-5}$ & $\cdots$ \\
\hline vanadiun & 381 & 0.023 & 155 & 1.25 & 6.2 & $\therefore 10^{-7}$ & $<10^{-6}$ & 11.4 \\
\hline $\begin{array}{l}316 \text { stain- } \\
\text { less stcel }\end{array}$ & 500 & 0.029 & 71 & 0.95 & 6.8 & $\cdot 10^{-3}$ & $\mid .4 \times 10^{-4}$ & 18.9 \\
\hline columb i iurn & 530 & 0.072 & 165 & 5.7 & 7.3 & $\therefore 10^{-10}$ & $\cdot 10^{-3}$ & 10.0 \\
\hline copper & 530 & 0.375 & 121 & 24.8 & $\cdots$ & $4 \times 10^{-2}$ & 0.032 & 1.3 \\
\hline n:olybdenum & 638 & 0.102 & 98 & 6.2 & 6.9 & $\cdot 10^{-11}$ & $\cdot 10^{-16}$ & 18.6 \\
\hline tastalum & 10,36 & 0,034 & 143 & 6.6 & 11.4 & $\therefore 10^{-15}$ & $.10^{-1: 5}$ & 18.9 \\
\hline $\begin{array}{l}\text { beryl lium } \\
\text { oxide }\end{array}$ & 148 & 0.092 & 513 & 1.7 & 2.3 & $.10^{-4}$ & $\cdot 10^{-8}$ & 13.4 \\
\hline
\end{tabular}

vai a at 700 a $K$. 

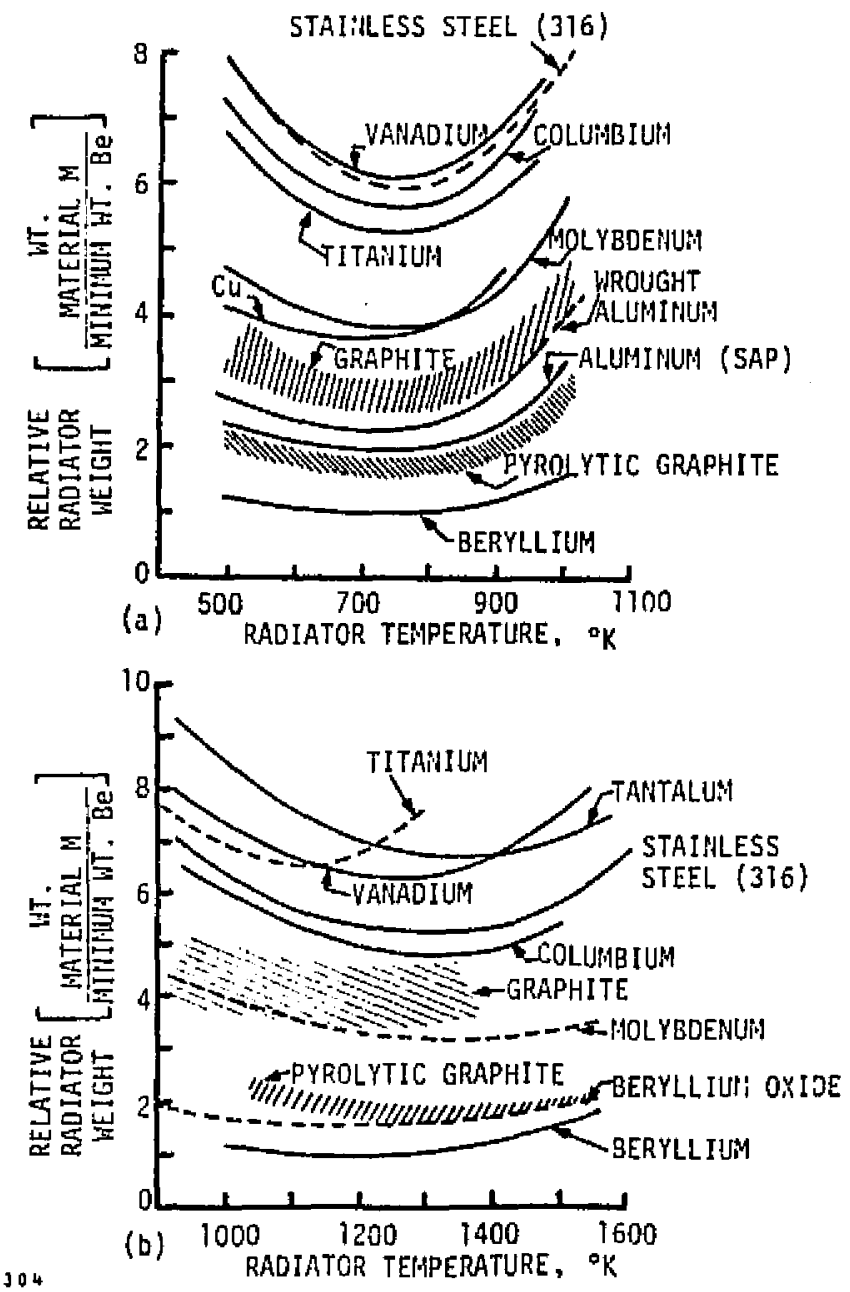

Figure 34. Radiator Fin and Tube ReTative Weight for a Turbo-Generator Rankine Cycle (Absolute Zero Effective Sink Temperature). (a) 1-MW potassium cycle, peak cycle temperature, $1370^{\circ} \mathrm{K}$; (b) $30-\mathrm{kW}$ mercury cycle, peak cycle temperature, $920{ }^{\circ} \mathrm{K}[63]$. 


\section{EVALUATION OF LASER HEAT ENGINE TECHNOLOGY}

The information presented in the previous section on heat engine concepts has been integrated with design criteria and mission constraints to select the best laser heat engine concept for a near-term experimental demonstration. A discussion of the design criteria and mission constraints is given below, followed by a summary of our evaluation of the engine concepts and a description of the best near-term heat engine recommended for experimental development. This evaluation also considered recommendations for a longer term development of larger, more efficient laser heat engine systems.

\subsection{Criteria for Optimum Design}

The laser heat engine concept to be chosen for experimental demonstration was specified to be consistent with near-term technology, defined as capable of being built and tested over the next two-year period. In particular, the concept had to utilize an available high power laser facility, be within the range of current high temperature, high strength materials and fabrication techniques, be compatible with an identified working fluid/absorber, and rely on experience with similar devices in area s where the design or operation are critical to the proper functioning of the experimental demonstration. Thus, the recommendation for an experimental program is dominated by very severe technology constraints.

Engine designs for space vehicles have very different and, in some cases, additional constraints. These constraints should be considerations at the laboratory demonstration phase in a general way, to be sure that there are no incompatible requirements; however, these restraints will require much additional study before a space optimized system can be conceived. In this category, we consider such requirements as the premium on low engine weight and volume because of the energy required to place the engine and space vehicle in orbit. Thus, the power per unit nlass developed by the engine may be a key parameter in measuring the desirability of different engines. The mission parameters may be specified in terms of a fixed power output.

Engine size must also be compatible with the function of the space vehicle. For example, a conumunication satellite may need multipolar antenna sites on the vehicle; an extremely bulky engine could hamper the placenlent of such antennae. Both power per unit volume and power per unit mass are variables which can be maximized. 
Engine cost, another important criterion, can be minimized by decreasing absolute weight and volume, by choice of materials and fabrication methods, and by reducing engine downtime through precision machining, careful sealing, and other design efforts. The cost of making the engine a closed cycle versus an open or "lossy" cycle, with the resulting space shuttle replacement of losses, can be assessed.

Continuous operation of the laser heat engine in space over at least a six-month period will probably be desirable. Engine concepts which require makeup fluids supplied periodically, for example, by the space shuttle, must meet the added constraint of resupply. The reliability of major components, such as basic window and wall structures, valves, pumps, etc. should be considered. Maintenance also is a serious consideration for an eventual application in space. Reliability criteria can generally be applied to different engine concepts to determine material and design choices.

High laser heat engine efficiencies are obviously desirable. Possible measures of efficiency are the ideal thermal efficiency of the cycle and the actual efficiency of each of the terms of the back-work ratio, where work inputs are those supplied to the engine parts to perform their tasks (e.g., to a compressor or to a piston in overcoming friction). Some devices show an increase in efficiency with size, especially where the losses are due to the amount of surface area relative to the working volume of the device. For example, turbines have power leakage past the blade tips, which decreases relative to the total power delivery as the turbine blade radius increases.

While a laboratory demonstration of the laser engine may be accomplished for a relatively small device, say 1-kll output fower, it will be necessary to know how the technology scales to higher powers. Some engine concepts aopear to have their usefui operating characteristics restricted to a rather narrow range of power outputs, and would be somewhat less desirable because of a lack of flexibility in meeting the power needs of a diversity of space vehicles. In particular, turbine engine efficiency scales better with higher power than piston engine efficiency for fixed compression ratio and peak cycle temperatures.

Several key attributes of the space environment are zero gravity, hard vacuum, occasional high energy particle fluxes from the sun, meteoroid bombardment, and solar radiation. A laser heat engine must be constructed to endure this environment, either by its design or by its mode of operation. For example, the solar heat load may be tolerated by allowing the engine surface to achieve an equilibrium reradiation temperature, or the vehicle may spin 
slowly to allow heated portions of the skin to cool off while facing away from the sun. Hard vacuum requires good seals to iteep in working fluids. The number and area of exposed seals therefore becomes an important feature of an engine design. Also, the vapor pressure of materials at high temperatures must be accounted for in this environment. Meteoroid bombardment requires additional armor, and hence weight, on all exposed surfaces where a puncture would involve a loss of operating capability (e.g., loss of working fluid). Zero gravity poses special problems for heat transfer and phase separation in working fluids, since "natural" convective processes are no longer operative. Engine types which require extra equipment or special modes of operation to get around these problems (e.g., for Rankine cycles) may incur a design penalty which must be recognized in comparing different classes of engines. General1y, high energy particle fluxes will cause radiation damage in exposed organic materials, such as lubricants, and in insulating and semi-conducting materials used in the electrical portions of the engine.

\subsection{Mission Constraints}

The on-board power requirements of NASA missions in the near future range from about 1 to $35 \mathrm{kWe}$. Generally, manned missions require the most power. The Apollo missions showed that manned missions could support life cycle processes with about one klle per person for short durations. Communication satellite systens will probably be the second largest non-military power users. A variety of these are planned at the moment, the most critical being the TDRSS (Tracking and Data Relay Satellite System) which will handle multiple data source inputs and relay them and their positions to ground or other satellite receivers. The TDRSS has a potentially much wider audience to serve than presently is planned for in its design. The problem, well recognized, stems from a data rate transmission 1 imit set, in part, by a solar energy power supply per satellite of $25 \mathrm{kWe}$ [66]. A larger power capability sized for each satellite would considerably enhance the effectiveness of TDRSS. The laser heat engine could conceivably supply more power for the TDRSS within the same size allotment to the present power system, because of the high thermal efficiency projected for the laser power system and smaller collector/ concentrator sizes.

Military missions could require much larger power levels, possibly to the multi-megawatt levels. These missions encompass communications, defensive, and offensive systems where increasing power levels determine the range and effectiveness of satellite capabilities [67].

Hence, we conclude that 1 to 35 KWe will support most of the benign near-earth space missions for the next decade or so, including satellite communications, the space-lab, and possibly even energy for the Long Duration Exposure Facility (LDEF), initially conceived of as a possible user facility. Tests for the solar. to-RF conversion and transmission for large solar power systems 
will need 60 to $500 \mathrm{kWe}$ RF power, and the Orbital Construction Demonstration Article (OCDA) will need approximately $250 \mathrm{kWe}$. Military and enlarged communication requirements may expand the power levels further.

An additional feature of many satellite systems and experiments planned for space shuttle orbit insertion is the trend toward design standardization. The shuttle will carry these packages to low earth orbit (LEO), and an intermediate-to-upper stage (IUS) booster will carry them to geosynchronous orbits (GEO) (approximately $38,000 \mathrm{~km})$. Standard space craft design will be either spinstabilized (e.g., always pointing in one direction, such as towards the laser power source), or two-direction stabilized, so as to receive and to send to separate targets. In one example, the power modules for each space craft have been standardized to the same volume: $48 \times 48 \times 18 \mathrm{in}$., excluding collectors and radiators [68].

Shuttle launch costs for smal1 units less than 200 pounds and 5 cubic feet range from $\$ 3,000$ to $\$ 10,000$ (i.e., $\$ 15$ to $\$ 50$ per pound) [69]. Larger packages can cost up to $\$ 160$ per pound to reach low earth orbit. Thus there is a premium on both weight and pre-orbit size: a deployable waste heat radiator and receiving optics would be a definite plus if these elements become a large part of the operative configuration of laser heat engines. Because of these cost and size constraints a convenient basis for comparing competitive laser heat engine concepts is on a per pound/cubic foot/hour-inorbit basis.

Lastly, Aerospace Corporation reports that the most cost-effective satellite maintenance system is to service satellites by orbit transfers from GEO to LEO and back again with the space shuttle, rather than to expend a malfunctioning satellite or to retrieve it for refitting on the ground. Their cost study suggests, as a general rule of thumb for laser heat engine maintenance procedures and reliability, that they be serviceable in orbit and have a reliability level consonant with periodic space shuttle flights [68].

In the context of mission constraints, it is worthwile reemphasizing some of the particular advantages of laser power compared to other satel]ite power systems. Probably the main competitors are solar power systells. Photovoltaic cells have been used extensively to provide electricity for space stations. The cost of such cells is decreasing with improved manufacturing techniques and more efficient ways of using them have evolved. However, their basic limitation is the relatively large collection area required because of the diffuse energy density of solar radiation at earth orbit.

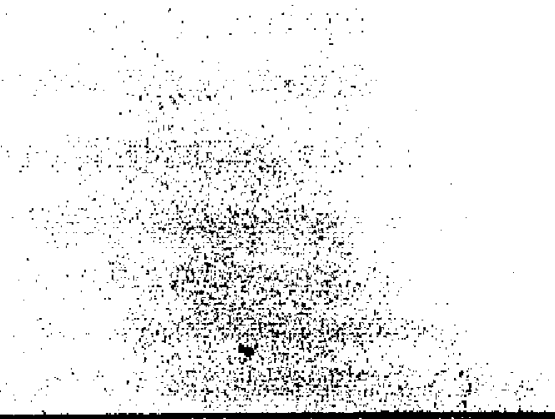


Solar energy can also be collected to power a thermal energy convertor, such as a Brayton cycle turbine, to make electricity onboard a satellite. In this case, the solar energy must be focussed to heat working fluids to a high temperature; yet, a collection area nearly as large as the photovoltaic collectors is required just to get the required amount of energy. The advantage of a thermal solar powered system is the relative ease in achieving high voltage through $A C$ power generation. Systems studies also show a cost advantage of thermal vs. photovoltaic power above a critical size measured in terms of output power [11].

With internal heating, a laser powered engine has the potential for being a much smaller system than a solar Brayton cycle. For example, solar Brayton efficiencies are on the order of 20 percent, whereas the target efficiencies for the laser heat engine are 50 percent, giving a 2.5 factor reduction in engine size and a 4-fold reduction in waste heat radiator size.

Furthermore, since the collector size for the laser heat engine depends only on the laser wavelength and transmission distance, there is a breakeven point in power required to run the engine, above which the laser heat engine collector will always be smaller than the solar collector. That is, the solar collector size must always increase with power required, whereas the laser collector is independent of the power required. The collector diameter for the laser heat engine, taken from Section 2.4, evaluated for $10.6-\mu$ laser radiation, is

$$
D_{c} \simeq 0.135 \sqrt{L(\mathrm{~km})} \text { in meters }
$$

where $L$ is the laser nower transmission distance. The breakeven power based on this equation and a 20 percent efficient solarpowered system is

$$
P^{*} \simeq 0.0076 \mathrm{~L}(\mathrm{~km}) \text { in kilowatts }
$$

At near-earth orbit $(L=160 \mathrm{~km})$, the breakeven power is $1.2 \mathrm{kwatt}$, and at geostationary orbit $(L=36,000 \mathrm{~km})$, the breakeven power is $275 \mathrm{kwatt}$. Thus, there should be a definite advantage for laser heat engines in the ten kilowatt power range for near-earth orbit application so far as collector size is concerned. Higher powered laser heat engines are required to maintain that advantage in geostationary orbits. 


\subsection{Selection Priorities}

In the light of the previous discussion, a choice of just three criteria was made to select the best laser heat engine for near-term demonstration. By limiting the number of criteria, a clear ordering in the priority in which they were applied could be obtained. The principle criteria for optimal design are, in the order in which they will be applied: technological availability, high efficiency operation, and a high specific power. These three criteria are not the only ones which can be applied to laser heat engines, but they do serve to give critical distinctions between the various contenders for the best laser heat engine. All of the engines we have examined, for example, appear to be capable of continuous operation for long times, and could be built for high reliability. Their relative thermal efficiencies and their capacity to exceed 50 percent overall efficiency, however, are in question. Similarly, some engine designs require sufficiently novel or unproven technology so as to cast doubt on their being built in the next two years. In view of the proposed schedule for a laboratory demonstration, utilization of current technology has been emphasized to select the most realistic laser heat engine. Most of the engines require common auxiliary equipment, such as space heat radiators, laser radiation collectors and focussing elements, generators, and energy storage units, so that the total system weights will be quite similar regardless of the heat engine itself. Hence, up to certain limits, the relative weights of different systems will be similar.

The application of these criteria for the choice of the best laser heat engine for longer term development is somewhat ditferent. First, the promise of higher operating efficiencies with technological development and with larger power outputs must be evaluated. Then, the criteria of technological availability must be relaxed to allow for developments, ongoing and planned, which promise to bear fruit in the next five to ten years. Further, space operational requirements must be considered more seriously. As it tirns out, the best engines selected for near and longer term application may be different and will probably serve different needs.

Each of the three Taser heat engine concepts developed in Section II was subjected to the selection criteria discussed in the previous paragraphs. First, an assessment of technological availability was made. Then, the overall system constraints on the engine component efficiency were evaluated. Finally, information on scaling and on specific power characteristics was applied to determine the performance of these concepts with increasing power output. The results of that evaluation follow in the next section. 


\subsection{Near-Term Technology}

The Stirling cycle is limited in the near future to material temperature of $1100^{\circ} \mathrm{K}$, or an ideal efficiency of 73 percent for an externally heated Stirling engine. Martini has studied Stirling cycle laser heat engines extensively from the viewpoint of attainable real cycle efficiencies, and concludes that an indicated efficiency of 55 percent should be possible. However, the Department of Energy (DOE) program suggests that the highest attainable Stirling cycle ef ficiency for a small (e.g., $1 \mathrm{~kW}$ ), externally heated engine will be closer to 35 percent, and then only in the 1985 time frame. Neither of these efficiencies meets the desired efficiency criteria (see Section 3.5).

A Beale-type, internally heated Stirling engine might permit higher internal operating temperatures without the development of new materials for the engine cylinder. We studied an 0tto-Stirling cycle in connection with a Beale-type engine to determine the ideal efficiency, technical feasibility, and materials/design problems. The isothermal heat addition portion of the ideal Stirling cycle was replaced by a constant volume heat addition by direct laser absorption in the working medium. This technique allows the gas temperature to rise above the hot wall temperatures, and thereby to increase the ideal thermal efficiency above that allowed by the wall temperature limits of an ideal Stirling cycle. However, to achieve sufficiently high themial efficiencies even for this hybrid cycle, the hot wall temperatures must be on the order of $1000^{\circ} \mathrm{K}$. Since the window must be cooled, a great deal of the energy at the hot end of the piston casing will be drawn off by the window coolant, vastiy reducing the real cycle efficiency. This design constraint was evidentiy encountered in recent experiments at NASA-Ames [70]. As indicated in Section 2.2.2, the Beale-type Stirling engine will probably not achieve sufficiently high efficiencies even at these temperatures, and has been excluded from immediate consideration.

All of the components of the energy exchanger/turbine laser heat engine are available now, except for an energy exchanger of proven high component efficiency. As will be shown in Section 3.6, the component efficiency must be high for high overall operating efficiencies. In Targe devices (e.g., larger than $250 \mathrm{~kW}$ ), comibined cycle designs can be employed which reduce the sensitivity of the overall efficiency on the energy exchanger efficiency.

The only laser heat engine which appears completely feasible in terms of near-term technology is the 0tto/Diesel cycle piston engine. The ordinary two-cycle combustion piston engine 
reproduces nearly all of the working conditions required of the laser heat engine. The walls are kept cool in the combustion case, primarily to keep the lubricants working properly. In the laser heat engine, the walls will a]so be kept cool to prevent reradiation losses through the receiving window. However, the piston should operate with dry bearings instead of a liquid lubricant, so that the window and the closed cycle heat exchanger are not fouled by the lubricant. Gas bearings can be designed for linear piston operation; crankshaft operation may be possible using solid lubricants having a low vapor pressure (see Section IV).

The working fluid temperatures and pressures for combustion driven 0tto and Diesel cycles reach values appropriate for efficient laser heat engine operation, so that no unusual design features are required, other than the window. We anticjpate being able to use a $\mathrm{CO}_{2}$ laser, a diamond window, and a 10.6- $\mu$ absorber diluted in argon as the working gas. Both $\mathrm{SF}_{6}$ and water vapor have been studied as absorbers. While $\mathrm{SF}_{6}$ distintegrates irreversibly above about 1500 ${ }^{\circ} \mathrm{K}$, it may be quite suitable for the first series of experiments if an intermediate goal efficiency is accepted. Water vapor appears to offer the greatest potential for long-term radiation absorption without chemical disintegration, but is also a good radiator, and consequently, the peak temperature is 1 imited by reradiation losses. Hence, eariy experiments with $\mathrm{CO}_{2}$ laser radiation may be inherently limited to modest system efficiencies on the order of 40 percent overa 11; however, fine tuning of the absorber and the engine mechanical design (rpm and lubrication) should be capable of increasing that efficiency to 50 percent.

\subsection{Efficiency}

A schematic of the laser heat engine system showing energy transfers between the individual major components is described in Figure 35. The component efficiencies are as noted in Figure 35. The combined system efficiency,

$$
n=\eta_{C} n_{E} n_{S} n_{G}
$$

is desired to be greater than 50 percent.

From our previous discussions, we can assign approximate upper values to each of these efficiencies, not including the heat engine component itself. At high rotational speeds, greater than 50,000 rpin, no speed transfer device is required (i.e., $n_{S}=100$ percent), and generator efficiencies can be quite high (i.e., $n_{G}=95$ percent). For 1 inear generators, if 


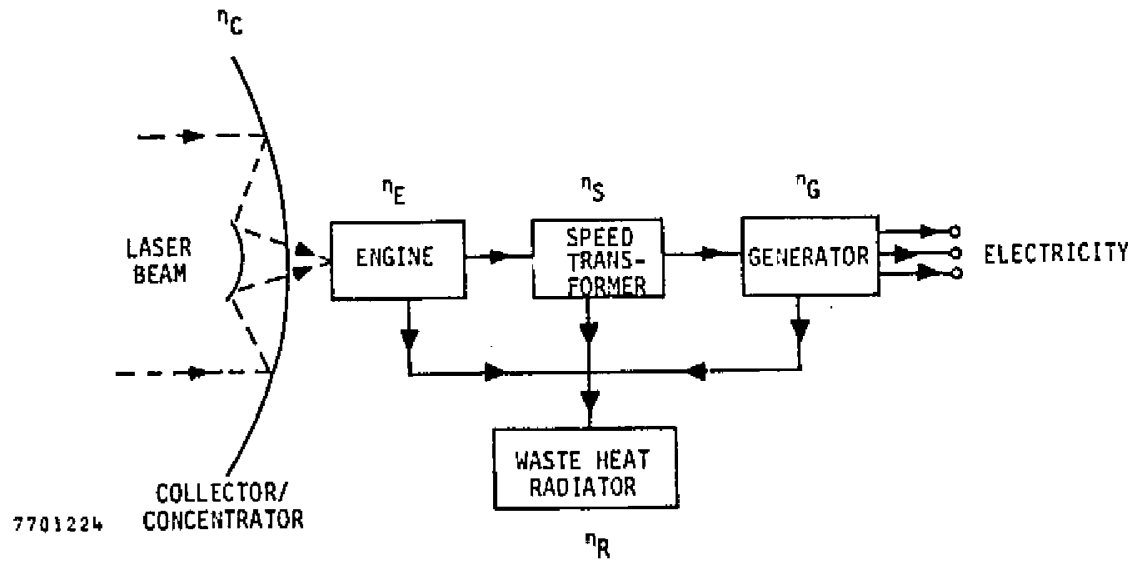

Figure 35. Laser Heat Engine System with Major Components and Efficiencies

the engine is operated at $3600 \mathrm{rpm}, \eta_{G}=90$ percent and $n_{S}=100$ percent; rotary generators with speeds less than 50,000 rpm may also be 1 inlited to $n_{G}=90$ percent and $n_{S}=100$ percent; at speeds less thar, $3600 \mathrm{rpm}, n_{5}=35$ percent may apply. Thus, there are three distinct cases:
a) $n_{S}=100$ percent
$\eta_{G}=95$ percent
Rotary generators driven by en- gine speeds greater than 50,000 rpm
b) $n_{S}=100$ percent
$n_{G}=90$ percent
Linear generators driven by en- gines at 3600 rpm; rotary genera- tors driven by engines with speeds between 3600 and 50,000 rpm
c) $n_{S}=85$ percent $\eta_{G}=90$ percent
Rotary generators driven by en- gines with speeds less than $3600 \mathrm{rpm}$

The collector/concentrator efficiencies can be as high as $\eta_{C}=98$ percent. Thus, cases a through $c$ place the following requirements on engine efficiency in order to achieve the desired 50 percent overall efficiency goal.

a) $\dot{\theta}>50,000 \mathrm{rpm}$

$$
\eta_{E}>\frac{0.5}{0.98(1.00) 0.95}=54 \text { percent }
$$


b) $50,000>\dot{8}>3,600 \mathrm{rpm}$

$$
\eta_{E}>\frac{0.5}{0.98(1.00) 0.90}=57 \text { percent }
$$

c) $3,600 \mathrm{rpm}>\dot{\theta}>0$

$$
n_{E}>\frac{0.5}{0.98(0.85) 0.90}=67 \text { percent }
$$

Clearly, there is a large penalty paid by operating the engine below $3600 \mathrm{rpm}$, assuming that $60 \mathrm{cycle} A C$ current is required. Most of the piston engines considered can operate in the range of 3600 to $6000 \mathrm{rpm}$, so that they can be classed with class b above; however, trictional losses and increasing wear will shorten the lifeime of such devices. For a six-month lifetime, the wear caused by greater rpm can probably be made a negligible effect. Frictional losses may be large enough to become an important cons,deration in deciding whether to operate an engine above $3600 \mathrm{rpm}$ or below. The only engine concept capable of operating in case a is the energy exchanger/turbine combination. Whatever gains are obtained by more efficient turbine-generator operation at high rpm must be balanced against the addition of another component in the energy transfer process, the energy exchanger, which has its own finite component efficiency.

Operating efficiencies for both the 0tto/Diesel cycle piston engine and for the energy exchanger/turbine engine were computed using component efficiencies. In effect, the work ratio information developed in Section II is integrated with actual operating experience of these engines to yield an efficiency which can be compared to the lower bounds required for the engine efficiency goal derived in this section.

The 0tto cycle efficiency results have been given in Section 2.2. Figure 17 showed a maximum efficiency of 60 percent is to bo possible for a peak cycle temperature of $1500^{\circ} \mathrm{K}$ and a compression ratio of 6 at low rpm; $1500^{\circ} \mathrm{K}$ represents a characteristic workirg fluid temperature that can be achieved for a near-term experiment using a $\mathrm{CO}_{2}$ laser. The 60 percent efficiency can be improved upon by driving the engine at higher rpm. At about $3600 \mathrm{rpm}$, the efficiency could marginally exceed the 57 percent value reguired for class b machines. Friction and pumping power losses will bring the efficiency down, making it difficult to reach an engine efficiency of 50 percent utilizing near-term technology. From this point of view, the 0tto/Diesel piston engine could make an exceedingiy interesting near-term experiment with a $\mathrm{CO}_{2}$ laser power source. With a $\mathrm{CO}$ laser (i.e., $3000^{\circ} \mathrm{K}$ peak temperature), the target overall efficiency of 50 percent may be reached more easily.

ORIGINAL PAGE IS OF PONR QUAJTTY 
As discussed earlier, the Diesel cycle is distinguished from the 0tto cycle by a longer heat addition time, approximating a constant pressure process as the piston extracts work, and consequently, a higher ideal efficiency from the same type of engine. The comparison of ideal cycle efficiencies in Figure 6 shows that the maximum work Diesel cycle may be 10 percent more efficient than the 0tto cycle at $1500^{\circ} \mathrm{K}$ peak temperature. This advantage is maintained in the actual operation of combustion engines where losses due to heat transfer, radiation, and friction are included. We would expect the same loss characteristics to carry over to the monatomic gas piston engine, implying that the Diesel cycle could be the best operating mode of the otto/Diesel laser heated piston engine. Results that are quoted for the otto cycle therefore represent conservative estimates, and hence a lower bound to the performance of this type of engine. The precise values for a Diesel cycle require a time-dependent combined heat addition and loss calculation beyond the scope of this study. Generally, we assume that 10 percent better performance is possible with the Diesel cycle.

Similar calculations were carried out for the energy exchanger/ turbine laser heat engine in Section 2.2.1. The results, as shown in Figure 14, revealed that utilizing the $\mathrm{CO}_{2}$ laser (i.e., low temperature case, $t: 5$ ) will not meet the minimum criteria on laser heat engine efficiency. However, at higher temperatures, which $\mathrm{CO}$ lasers can produce $(\tau=10)$, the turbine/energy exchanger engine has an overall efficiency of 56 percent, even for the small $1-\mathrm{kW}$ size where the system is not very efficient, exceeding the 54 percent goal required of class a laser heat engines. As shown below, the efficiency can be as high as 65 percent for turbine/energy exchanger engines of 100 kilowatts and larger.

\subsection{Scaling to Larger Powers}

\subsubsection{Efficiency-Power Dependence}

The principal advantages of gas turbines compared to piston engines become apparent when larger power outputs are considered. It is well known that it is difficult to obtain high component efficiencies in sma11 turbines and compressors. As the size of the device increases with increasing power, the turbine and compressor losses decrease as the inverse square root of the power. In contrast, piston engine efficiency can be maintained down to quite low powers, on the order of 10 $\mathrm{kW}$, before size scaling effects begin to reduce the engine performance appreciably. These scaling trends are shown in Figure 36 , which is a composite of both theoretical and empirical data. Our approach in constructing Figure 36 has been to use the empirical data as the base case, and to build on that data using theoretical estimates of efficiency.

The combustion data (Diesel piston engine and vehicular gas turbine with regeneration) were taken from reviews of engine performance carried out by Schnel1 [70] and Jaunotte [71]. Points for the operation of rare gas $(Y=5 / 3)$ turbines designed for electric power generation in space vehicles illustrate the increase in efficiency due to 


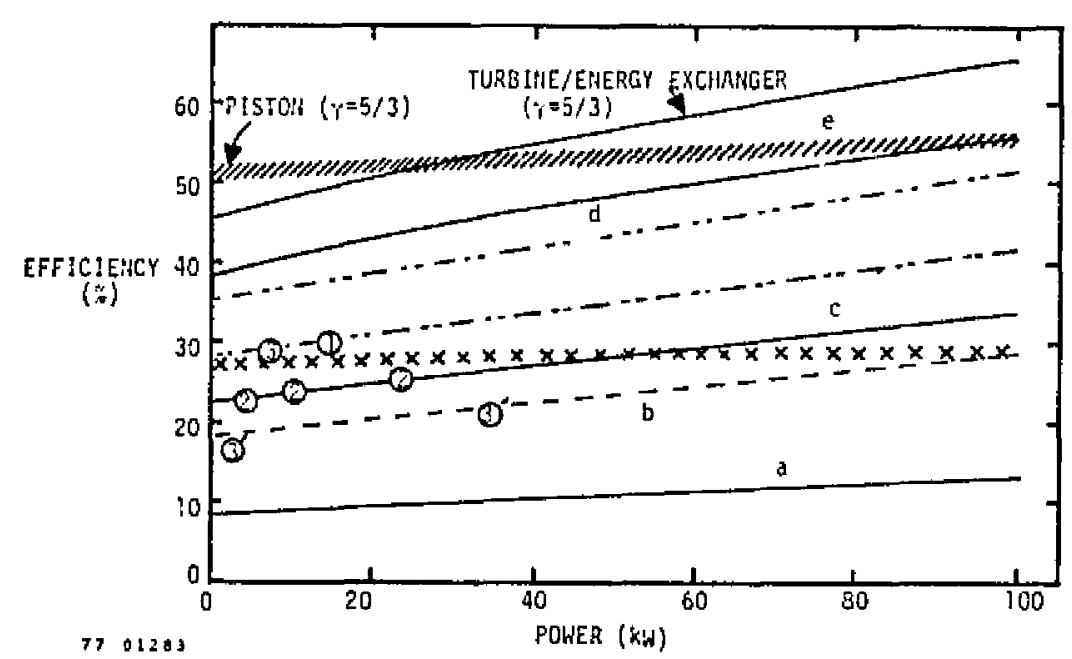

\footnotetext{
POIITS:

(1) Solar Brayton (Arqunj [1] O Isotope Brayton $\{\mathrm{Xe} / \mathrm{He}$ ) [20]

O Solur Brat ton [72] $\mathcal{O}$ Snap 2 and 8 Brayton (xe/he) [20j

/// Diesel Engires, $y=5 / 3$ (Argon) $x x x$ Diesel Engine, $y=1.24$ (Combustion: [71]

CURVES: a) Conbustion cas Turbines (for Vehicles) with Regeneration; $T_{\max }-950{ }^{\circ} \mathrm{K}$.
$Y=1.24$ [7i]

b) Argon Gas Turbines (Closed Cycle) with Regeneration; $T_{\max }=950{ }^{\circ} \mathrm{K}$. $T_{\min }=345{ }^{\circ} \mathrm{K}, \gamma=5 / 3$

c) Helium-xenon Gas Turbines (Closed Cycle) with Regeneration: $T_{\text {mats }}$ - $1080 \mathrm{ag}$, $T_{\text {min }}=300^{\circ} \mathrm{K}, y=5 / 3$ (Ranje of Performance Due to Component metficiencies)

d) Helfun-Xenon Gas Turbine/Energy Exchanger with Regeneration: $T_{\text {indx }}$ $3000^{\circ} \mathrm{K}, T_{\min }=300^{\circ} \mathrm{K}, \gamma=5 / 3$

e) Same as (d) and with Intercooled Three-stage Compression (Range of Performance Que to Component Efficiencies)
}

Figure 36. Scaling of Prime Mover Efficiency with Power

the change in $\gamma$ from the combustion case, $\gamma \sim 1.24$. The observed ratio of efficinncies for these two cases corresponds very nearly to the ratio of ideal Brayton cycle efficiencies for $\gamma=5 / 3$ and $\gamma=1.24$, with a turbine pressure ratio of 2 .

The improvement in piston engine performance available through the use of a monatomic gas has been estimated from the combustion Diesel case, by assuming that the efficiency can be multiplied by the ratio of ideal efficiencies for $\gamma=5 / 3$ and $Y=1.24$. A compression ratio of 17 was assumed to apply at all power levels. The results are represented in figure 36 by the hatched line, ranging from an efficiency of approximately 52 percent at low powers to 57 percent at $100 \mathrm{~kW}$. These values correspond well to the theoretical values derived in Section 2.2.2.

With the addition of an energy exchanger, the peak cycle temperature can be raised to $3000^{\circ} \mathrm{K}$, approximating the temperature achievable with a co laser. Line $d$ in Figure 36 corresponds to the increase in turbine cycle efficiency obtained by raising the peak temperature from $1080^{\circ} \mathrm{K}$ (top of band $\mathrm{c}$ ) to 3000 ${ }^{\circ} \mathrm{K}$. Line $d$ and the top of $c$ are related just by the ratio of 
Carnot efficiencies for 3000 and $1030^{\circ} \mathrm{K}$. If an intercooled, threestage compression cycle is also used, as discussed in Section 2.2.1, then the turbine/energy exchanger engine can boost its efficiency from approximately 42 to 55 percent. Thus, the top of band e corresponds to the intercooled system, where the 42 percent efficiency performance at $40 \mathrm{~kW}$ on line d was scaled to 55 percent on line e. These calculations have assumed an energy exchanger efficiency of 85 percent. Lower component efficiencies account for the spread in band $\mathrm{e}$.

It appears from the preliminary scaling results that the turbine/energy exchanger laser heat engine will be significantly more efficient than the piston laser heat engine for powers greater than approximately $100 \mathrm{~kW}$. With improvement in energy exchanger component efficiencies, this cross-over power may drop to as low as $25 \mathrm{~kW}$.

\subsubsection{Size-Power Dependence}

A basic difference in size exists between turbine and piston engines delivering the same power output and working between the same cycle temperatures, if an energy exchanger is used with the turbine. Two major factors enter the difference in size: duty cycle and pressure ratio. Turbines have a 100 percent duty cycle because they deliver power continuously, whereas, at best, a piston engine delivers power only every other stroke, or, 50 percent of the time. This difference tends to make turbines smaller than piston engines. On the other hand, piston engines are efficient with a single cylinder sustaining large peak-toambient pressure ratios, whereas several turbine stages may be required to cascade the peak pressure down to ambient pressure conditions. This tends to make piston engines smaller than turbine engines.

By considering the two types of engines to use materials having approximately the same mass density, the ratio of turbine engine mass to piston engine mass can be written as

$$
\frac{W_{T}}{W_{p}}=\frac{\pi r_{T}^{2} L_{T}}{\pi r_{p}^{2} L_{p}}=c_{o}\left(\frac{L_{T} P_{T}}{\pi r T}\right) \cdot\left(\frac{\pi p}{r_{p}}\right)^{3 / 2}
$$

where the subscripts $T$ and $p$ refer to turbine and piston, respectively, $r$ and $L$ are radius and length, $P$ and $\pi$ are the power output and pressure ratio, and $C_{0}$ is a constant. $r_{T h i s}$ expression reflects the fact that the power output for a turbine is proportional 
to the turbine blade area and to the pressure ratio. The power output from a piston engine is proportional to the $3 / 2$ power of the piston displacement volume (as discussed in Section 2.2.2) and to the pressure ratio.

The above expression can be evaluated for $P_{T}=P_{p} \equiv P$, and for the particular sizes of turbines and piston engines capable of yielding a given power output of one kwatt. A piston engine operating with a pressure ratio of 40 at $500 \mathrm{rpm}$, with a bore of $2 \mathrm{r}_{\mathrm{p}}=$ $7.6 \mathrm{~cm}$ and cylinder length $L_{T}=20 \mathrm{~cm}$, can produce $1 \mathrm{kwatt}$. A150, a four-stage turbine with one energy exchanger and three intercooled compressors, each component $1.4 \mathrm{~cm}$ in radius and $1.4-\mathrm{cm}$ long (i.e., $L_{T}=8 \times 1.4$ ), operating with a turbine stage pressure ratio of 2.05 , can also produce $1 \mathrm{~kW}$. Thus, the above expression yields

$$
\frac{W_{T}}{W_{p}}=0.23
$$

and

$$
c_{0}=1.63 \times 10^{-4} \frac{(\mathrm{kW})^{1 / 2}}{\mathrm{~cm}}
$$

Hence, we may conclude that a definite power-to-weight ratio advantage exists for the turbine/energy exchanger combination for small power outputs. This advantage becomes even more pronounced as the power increases, since $W_{T} / W_{p}$ varies as $p-1 / 2$.

Both the generator and radiator masses can be assumed to vary roughly with $P$ to the first power. However, the high speed turbine generators can aiso be more compact and i ightweight compared to slower speed generators used with piston engines, because the high speed generators are more efficient in themselves, irrespective of the engine efficiency. The increase in radiator weight with increasing power will be offset somewhat by the rise in engine efficiency associated with increasing power. Hence, the turbine will again show some advantage over the piston engine, since its efficiency can be greater for powers above 50 to $100 \mathrm{~kW}$.

In conclusion, the turbine/energy exchanger should have a definite specific power (i.e., power-to-weight ratio) advantage over the piston engine, even at very small sizes. For all of the system components whose weight depends on power, this advantage increases 
with increasing power. Without a complete weight optimization, it is not possible to determine what the total weight advantage of the turbine/energy exchanger power systems is, but it appears that the engine itself can be up to four times lighter than a piston engine of the type described for the same power output.

\subsection{Summary of Evaluation}

The evaluations of cycle efficiency, near-term technology, power-to-weight ratio, scaling to high power, and the outlook for each of the engines is summarized in Table 16. On this basis, the 0tto/Diesel cycle piston engine has been chosen as the best laser heat engine for near-term demonstration.

Table 16

Summary of Evaluation

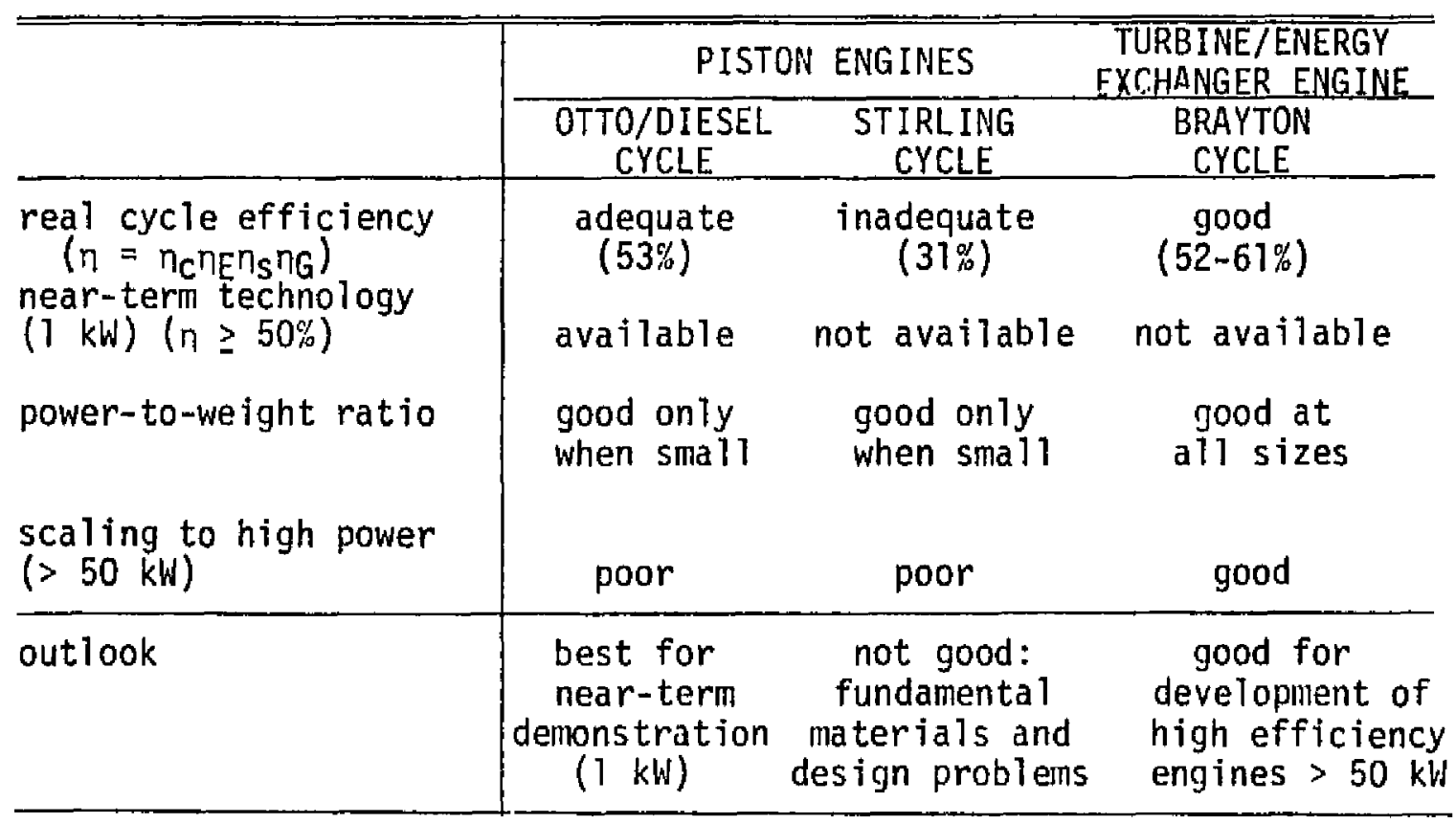


The calculations for the overall system efficiency shown in Table 16 assume that the Otto/Diesel engine is a class (b) laser heat engine $\left(\eta_{S}=1.0, \eta_{G}=0.90\right)$ with $n_{E}=0.60$, that the Stirling engine is also a class (b) engine with $n_{E}=0.35$, and that the turbine/energy exchanger engine is a class (a) laser heat engine (i.e., $n_{S}=1.0, n_{G}=0.95$ ) with $n_{E}$ ranging from 0.56 to 0.65 . For each case in Table $16, \eta_{c}=0.98$.

With current technology, the Stirling cycle piston engine does not achieve the desired 50 percent or greater overall efficiency. Further, the prognosis for future development to achieve that level of efficiency is not favorable, since the refractory materials needed for high temperature operation will also lose heat rapidly by conduction. The turbine/energy exchanger laser heat engine appears to have good efficjencjes at higher powers but requires component development, particularly at the $\rceil-k W$ power level to achieve 50 percent or greater efficiencies. Further, it can only achieve its potential using a co laser source which, although within the state-of-the-art, is not available. The outlook for future development for this concept is good since the technology for energy exchangers is under ai:tive pursuit within the DOE, and consequently, would allow efficient laser heat engines of this type to be built. 


\section{THE DEMONSTRATION EXPERIMENT}

Based on the evaluation presented in the previous section, a baseline laser heat engine system has been conceived which has a good chance of meeting all of the objectives set out for this study. This baseline concept has subsequently been simplified into a demonstration experiment which is designed to illuminate the unique and unproven technology comprising this system, i.e., the laser engine itself. This section first describes the baseline system and then describes the demonstration program proposed for the engine.

Although this baseline laser heat engine is not the ultimate in efficiency and power scaling potential, it is a system which could well fulfill near-term missions while longer range technology is developed.

\subsection{Description of the Baseline \\ Laser Heat Engine System}

A two-stroke Otto/Diesel piston engine is recommended as the baseline system and demonstration laser heat engine. The idea 1 and actual advantages of coupling the resonant absorption process with an Otto/Diesel cycle were established in Section III. A laboratory demonstration experiment is the next step toward establishing its suitability for space applications. The design philosophy followed in developing the criteria for selection of the 0tto/Diesel concept has been to use only existing technologies for the basic engine components. These components have been combined in a self-consistent way to establish the "baseline" engine system sketched in Figure 37, which shows each of the major engine components in a stand-alone arrangement. Clearly, these components could be arranged different;y for different satellite applications, to allow for the objectives of the satellite mission. However, certain aspects are fixed, such as the relative orientation between the collector/concentrator and the waste heat radiator, which has been chosen so as to minimize thermal distortions in the collector bowl. Also, the generator should be sealed onto the engine casing so that a separate generator coolant circuit and seaing of a drive chain is not required.

From this baseline concept, a rne-kilcwatt engine and its possible construction material and ope zting parameters are suntmarized below in Table 17. 


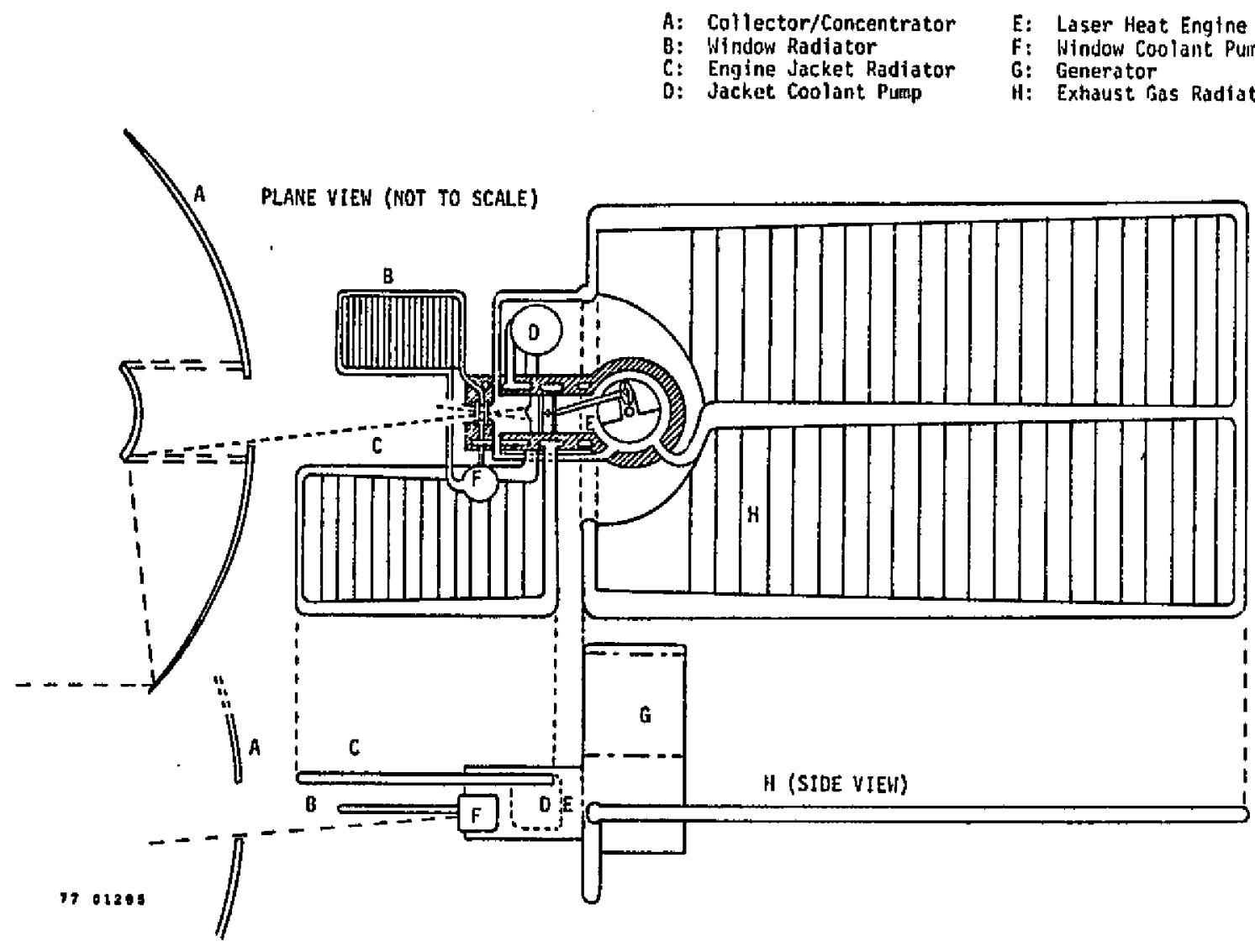

Figure 37. Baseline Laser Heat Engine System

Table 17

Laser Heat Engine Baseline Design

\begin{tabular}{|c|c|}
\hline $\begin{array}{l}\text { Engthe Type: } \\
\text { Power Dutput: } \\
\text { Operation: }\end{array}$ & $\begin{array}{l}\text { Otto/Diesel piston engine } \\
1 \mathrm{kH} \\
2 \text { strokes with crankcase } \\
\text { compression } 500 \text { rpm }\end{array}$ \\
\hline $\begin{array}{l}\text { Bore: } \\
\text { Stroke: } \\
\text { Compression Ratio: } \\
\text { Laser Wavelength: } \\
\text { Peak Cycle Pressure: } \\
\text { Working Flujd: } \\
\text { Aosorption Coefficient: } \\
\text { Minimum Cycle Pressure: } \\
\text { Peak Cycle Teap.: } \\
\text { Minimum Cycle Temp.: } \\
\text { Window: } \\
\text { Unattended Operation: }\end{array}$ & 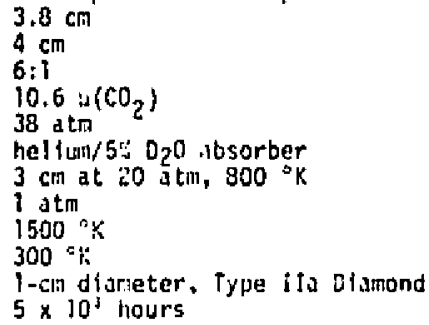 \\
\hline
\end{tabular}

Other Features

1. Crankcase \& Cylinder Jacket cooling: hellum

2. Window Construction: double windows, simply supported, each 1-mm thick and face-cooled with high pressure helium

3. Seals, Bearings, and Lubrication:

a. Piston Sealing: carbon impregnated rings on

b. Piston Rod a Pin: sast iron or harded chronte

with fluorcarbon grease

c. Main Dearing: sealed wi th roller bearings packed

4. Sizes: with fiugrcarbon grease
d. Collector/concentriter: îtis diateter.
b. Piston Engine: $45 \mathrm{~cm}^{\mathrm{s}}, 2^{2} \mathrm{~kg}$ (met. kapton)
c. Generator: $5 \mathrm{~kg}$
d. Radiator: $1.3 \mathrm{~m}^{2}, 3.6 \mathrm{~kg}$
e. Total Hass: $12.6 \mathrm{~kg}$
5. Figures of Merit: Therral lefficiency 5 :

Overall Efficiency
Specific fower


In the paragraphs that follow, the details of each of the important design features will be discussed. The baseline engine design is presented to illustrate the existing potential for progress toward a simple and efficient space energy conversion scheme. It represents a point design and is by no means intended as an optimum. However, the design serves tiwo important purposes: first of al1, a laboratory demonstration device can be derived from it for which the emphasis on mechanical simplicity will help investigators distinguish between losses associated with the resonant absorption process from those of other sources, and secondiy, it will serve as a framework for discussion and a point of departure for further innovation.

\subsubsection{Cycle Characteristics}

The proposed engine will be operated at slow speeds $(500$ rpm). Two-stroke engines have the advantage of twice as many power strokes as four-stroke engines. Thus, they have smoother operation and require a smaller flywheel than a four-stroke engine. The work needed to overcome the friction of the exhaust and suction stroke is saved compared to a four-stroke engine. The exhaust gases are scavenged by a fresh charge of cold gas, injected at a slightiy nigher pressure through the side ports in the opposite side of the cylinder at bottom dead center. Some compression of the injected gas is required. This can be accomplished either in the crankcase beneath the piston on the downstroke, or by a separate compressor outside of the cylinder. Crankcase compression has been chosen for closed loop operation of the baseline design.

Cooling of the engine head cylinder and crankcase can be accomplished by circulating high pressure helium in the baseline design engine jacket. In the demonstration experiment, external compression of scavenging gases may be used and a liquid coolant, such as water, may be employed to cool the engine jacket.

The laser window must be able to transmit $\mathrm{CO}_{2}$ radiation. It must also be able to withstand the cyclic stress associated with the gas pressure excursion. The double-layer design shown in Figure 37 was identified as the preferred configuration. The two windows with simply supported edge conditions witi each withstand equal differential pressure increments with face cooling provided in the space between. Estimates of the heat transfer rates and coolant pumping power show that the windows can be maintained at a temperature below $700{ }^{\circ} \mathrm{C}$. Thermal stresses due to the temperature excursions in the gas were investigated and found to be well within the constraints imposed by the window materials. 
The list of candidate window materials is 1 imited by the above design constraints. Fortunately, Type Ila diamonds have the requisite properties. A list of properties for diamonds is given in Table 18. The high tensile strength of diamonds permits individual $1-\mathrm{cm}$ diameter windows as thin as $1-\mathrm{mm}$ each to withstand a 20-atm pressure differential.

Table 18

Properties of Natural Diamonds

(taken from Ref. 39).

\section{Density}

Volumetric Heat Capacity

Elastic Modulus

Linear Thermal Expansion

Thermal Conductivity for II a

Refractive Index

Temperature Dependence

Brewster Angle

Absorption

Tensile Strength

Themal Diffusivity

$$
\begin{aligned}
& \rho=3.52 \mathrm{~g} / \mathrm{cc} \\
& \rho_{c}=1.8 \mathrm{j} / \mathrm{cc} \mathrm{K} \\
& C_{1}=9.5 \times 10^{12} \mathrm{~d} / \mathrm{cm}^{2} \\
& \mathrm{z}=1.2 \times 10^{-6} \mathrm{~K}-1 \text { at } 27{ }^{\circ} \mathrm{C} \\
& k=20 \mathrm{~W} / \mathrm{cm} \mathrm{K} \text { at } 30^{\circ} \mathrm{C} \\
& n=2.39 \\
& d n / \mathrm{dT}=1.9 \times 10^{-5} \mathrm{~K}^{-1} \\
& \theta=67 \text { degrees } \\
& \beta=0.01 \text { to } 0.3 \mathrm{~cm}^{-1} \text { for IIa } \\
& F=1.8 \times 10^{10} \mathrm{~d} / \mathrm{cm}^{2} \\
& k / \rho C=11 \mathrm{~cm}^{2} / \mathrm{sec}
\end{aligned}
$$

Type Ila diamonds are found naturally in only one mine in Africa but can also be created synthetically. A naturally occurring diamond, $1-\mathrm{cm}$ diameter across the face, might cost between $\$ 8,000$ and $\$ 25,000$ depending on its colors and fautts. Synthetic IIa diamonds can be made only as large as $0.5 \mathrm{~cm}$ in diameter. Some attempts have been made to join several diamonds together with a bonding compound and high pressures, but these have not been successful to date. Data on absorption and other material properties is well known, and recently, $\mathrm{CO}_{2}$ laser tests conducted at AVCO suggest that a steady flux of $1 \mathrm{MU} / \mathrm{cm}^{2}$ can be sustained by a diamond window.

For purposes of the experiment, it may be more appropriate to use a cheaper substitute which would absorb more of the laser radiation, so lonn as the window absorption is discounted when computing actual engi e performance. Two 4-mm thick coated zinc sulfide discs could withstand the same pressure differential, and would transmit 70 percent of the incident laser radiation. Zinc sulfide is sold commercially as Irtran, and is available at a fraction of the cost of diamond wi. dow. The disadvantage of this approach is the need for greater laser power and more extensive window cooling to perform the experiment. 


\subsubsection{Lubrication}

Lubrication is a critical problem in any laser heated piston engine design. The necessity of maintaining optimum absorption characteristics of the engine, working fluid, and absorber preciudes the use of conventional lubricating methods. Three design options were considered in addressing this problem. They are shown in Table 79.

\section{Table 19}

\section{Design Options for Engine Lubrication}

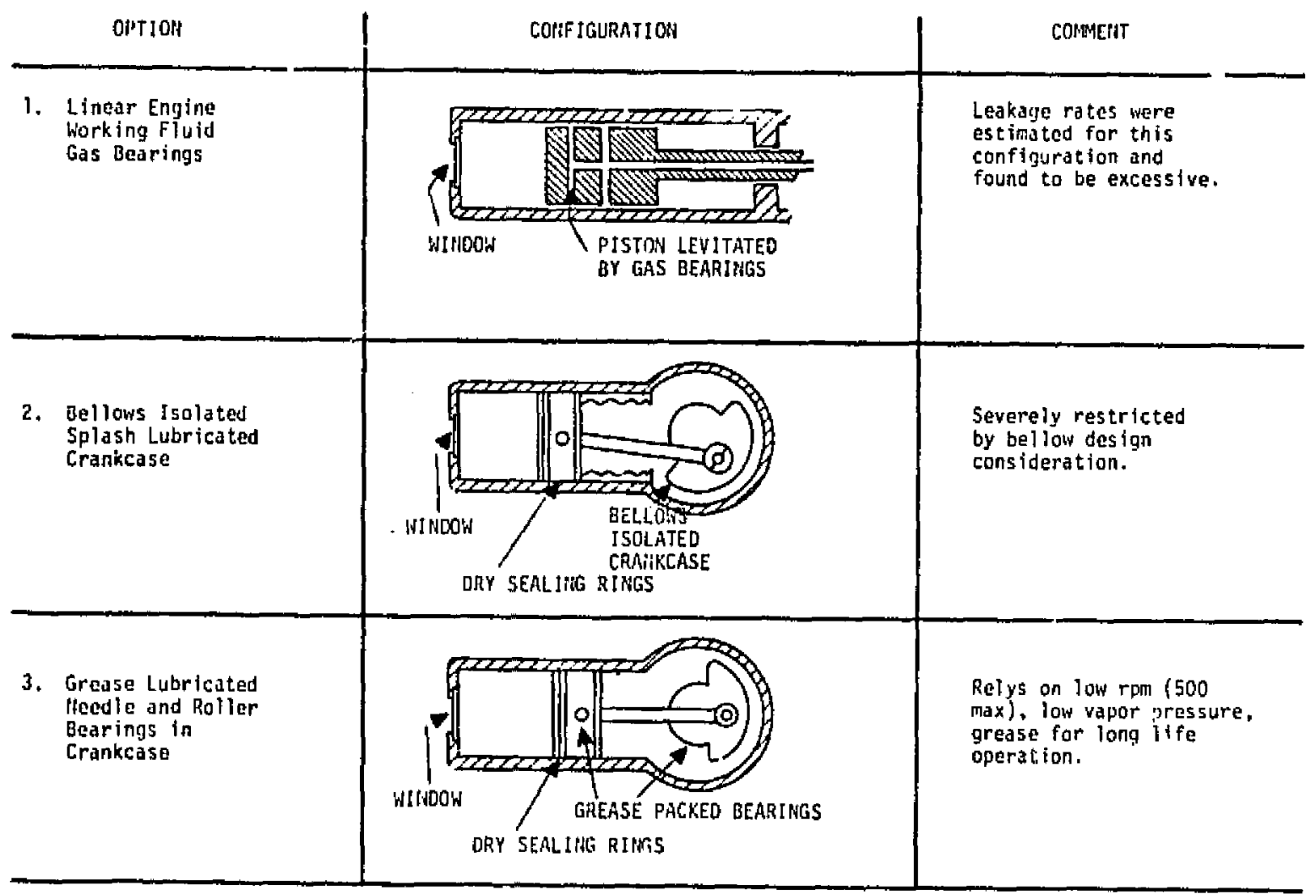


The first, and apparently the cleanest, is a linear configuration which would power a linear al ternator. This device relys on gasdynamic bearings to lubricate the cylinder piston interface, connecting rod, and linear alternator plunger. A serious drawback to this approach is the leakage loss encountered at normal airbearing radial clearances with required piston pressure differentials (e.g., 30 to $40 \mathrm{~atm})$.

The leakage loss for such a device lies between the limits of adiabatic flow with friction, and isothermal flow with friction. Calculation of these limiting cases, together with a comparison of the flow time to the thermal conduction time for the fluid in the clearance space gives large leakage losses for a 30-atm pressure differential and realistic radial clearances of 0.00125 $\mathrm{cm}(0.5 \mathrm{mil})$ to $0.0025 \mathrm{~cm}$ (1 $\mathrm{mil})$.

The second option uses a mechanical bellows to isolate a splash lubricated crankcase. Bellows would be required to isolate the cylinder from the crankcase in this scheme. However, bellows with the required stroke and $L / D$ suffer from "squirm" at speeds in excess of $300 \mathrm{rpm}$. It would aiso introduce an unknown loss contribution from friction due to rubbing on guides surrounding the bellows. This approach was judged marginal based on the above considerations.

The third design, chosen for the baseline version, uses roller bearings for the rod-piston, rod-crank, and ma in bearings. These components will be backed with fluorcarbon grease and should provide the necessary lubrication for the 5,000-hour service life set forth in the objectives without the necessity for elaborate crankcase isolators.

\subsubsection{Piston Sealing}

The problems of lubrication and sealing are intimately tied to one another, as can be seen from the previous discussion. The problem of piston sealing for the laser 0tto/Diesel cycle engine is identical in many respects to that encountered in the 0tto/Diesel combustion engine. Piston rings in both are exposed to high temperature gases. This precludes the use of organic fluorocarbon materials, such as are used in air compressors and Stirling cycle work pistons. Carbon seals impregnated with various additives have been used in "dry" applications. Pure carbon seals have excellent wear and self-lubrication rroperties, but generally require the presence of small quantities of water, which are normally available from the ambient atmosphere. It is interesting to note that by utilizing deuterated water as an absorber in combination with pure carbon seals, it may be possible to capitalize on this symbiotic relationship. Carbon and graphite seals impregnated with various elements and compounds have been developed 
to perform in the completely dry environments of high altitude and outer space with greatly improved friction and wear characteristics over pure carbon. Data are avajlable on wear rates for carbon running at various speeds and loads for various mating surface materials [73]. The wear ratio, $\omega$, is the increment of material removed per unit of length rubbed during testing. Table 20 shows some typical and "best case" results.

Table 20

Some Carbon Wear Rate Data

\begin{tabular}{rcccc}
\hline \hline $\begin{array}{c}\text { TEMPERATURE } \\
{ }_{\mathrm{F}}\end{array}$ & $\begin{array}{c}\text { MATING } \\
\text { MATERIAL }\end{array}$ & $\begin{array}{c}\text { RUBBING SPEED } \\
(\mathrm{ft} / \mathrm{min})\end{array}$ & $\begin{array}{c}\text { CONTACT PRESSURE } \\
(\mathrm{psi})\end{array}$ & $\begin{array}{c}\text { WEAR RATI0 } \\
(\mathrm{in.} / \mathrm{in} \text { ) }\end{array}$ \\
\hline 70 & hard chrome & 440 & 20 & $5.0 \times 10^{-10}$ \\
800 & $\mathrm{Si}_{3} \mathrm{~N}_{4}$ & 279 & 15 & $1.3 \times 10^{-10}$ \\
250 & chrome & 442 & 20 & $1.2 \times 10^{-12}$ \\
\hline
\end{tabular}

Increasing the contact pressure, $\mathrm{p}$, increases the wear ratio with

$$
\omega \sim p^{1 / 2}
$$

For a piston ring, the contact pressure is of the order of the pressure drop across the ring. If the "best case" data shown above are used in coijunction with baseline engine parameter's, the total material removal, $W$, in a $5 \times 10^{3}$ hour operating interval can be estimated by

$$
W=1.2 \times 10^{-12} \frac{\mathrm{in} .}{\mathrm{in} .} \times 10 \frac{\mathrm{in} .}{\text { cycte }} \times 10 \frac{\mathrm{cycle}}{\mathrm{sec}} \times 3600 \frac{\mathrm{sec}}{\mathrm{hr}} \times 5 \times 10^{3} \mathrm{hr}
$$

or

$$
W=0.002 \text { in. }
$$

For 5 rings with equal pressure increments across each, and a 20 atm average pressure, the effect of pressure would increase the above value to

$$
W^{*}=W(P-/ P)^{1 / 2}=0.002 \text { in. } \times\left(\frac{20 a \operatorname{tin}}{5 \text { rings }} \times 15 \frac{p s i}{a t m} \times \frac{1}{\frac{20 p s i}{r i n g}}\right)^{1 / 2}
$$


Thus,

$$
W^{*}=0.0035 \mathrm{in} .
$$

Although there are other factors, such as inertial loading, which may contribute to additional wear, this result provides a bas is for optimism in applying carbon seal technology to this difficult problem area.

Perhaps the most extensive investigation of sealing materials under conditions comparable to those found in the laser heated 0tto/ Diesel engine was conducted by Curtiss Wright to improve the apex seals for the Wankel engine [74]. Exhaustive testing of the friction and wear properties of a broad range of materials produced seals with greatiy improved characteristics. Of particular interest were several combinations which were not optimum from a cost point of view for the Wankel application, but exhibited exceptionally good wear and friction properties. It appears that a further design effort should be pursued within this on-going area of seal technology to establish which seal materials wear best in the laser heat engine environment.

\subsubsection{Window and Block Cooling}

To avoid problems with frozen coolant when the engine is not operating, we recommend that hel lum be used for cooling the windows. A sillall amount of electrical energy must be stored to run the helium compressor during laser engine start-up (start-up operation is discussed in more detait in Section 4.2). By blowing the helium across the window face under high pressure (i.e., 10 to $15 \mathrm{~atm}$ ), rather good heat transfer film coefficients can be obtained to limit the film temperature drop to approximately $50^{\circ} \mathrm{K}$. The $50^{\circ} \mathrm{K}$ drop in temperature insures that the outer surface of the inner window can be kept cool, and the inner window surface will then be at

$$
\mathrm{T} \cong 540^{\circ} \mathrm{K}
$$

for diamond windows at peak working fiuid temperatures of $1500^{\circ} \mathrm{K}$, assuming that the working fluid is helium with

$$
h=45 \frac{\text { watts }}{m^{2}+k^{\prime}}
$$

This temperature is well within the melting and stress limits of diamonds. The melting point of diamonds is above $3500^{\circ} \mathrm{C}$. As discussed, hel ium can be used to cool the engine block. Approximately 30 percent of the incoming power must be disposed of through engine jacket cool ing. 
In the demonstration experiment, water cooling can be used with a conventional radiator if once-through cooling is not feasible. However, pumping power and the heat carried away by the water must be monitored carefully to determine the cold engine performance. In this regard, an attempt to simulate the coolant pressure drop corresponding to the space radiator should be made.

\subsubsection{Piston Heat Transfer}

In small engines, the absorption mean free path may be somewhat longer than the top dead center piston distance from the window. To insure complete absorption of the laser radiation in the working fluid, both the top of the piston and the inside upper surface of the cylinder should be reflective. An evaporated gold coating can be made 99.4 percent reflective; as long as these surfaces do not overheat, they should retain that reflectivity. Heat transferred to the cylinders is carried away by the engine jacket coolant. Heat transferred to the piston must be conducted through the piscon to the crankcase gases. For this purpose, the piston should be made thin and corrugated on the surfaces exposed to the crankcase, so that good cooling is achieved.

While the gold coating may reflect most of the $\mathrm{CO}_{2}$ laser radiation, the hot gases will transfer heat quickly to the piston. With a $1500^{\circ} \mathrm{K}$ helfum working fluid, the forced convection heat flux is 5.4 watts $/ \mathrm{cm}^{2}$. If the piston is $0.5-\mathrm{cm}$ thick aluminum alloy, and with an effective heat transfer coefficient of $h=40$ watts $/ \mathrm{m}^{2}{ }^{\circ} \mathrm{K}$ on the underside of the piston, the piston temperature will be approximately $490^{\circ} \mathrm{K}$. To achieve these low temperatures, one must also account for cooling by the scavenging gas at bottom dead center. The piston temperature is sufficiently far from the melting tenperature of gold $\left(1340^{\circ} \mathrm{K}\right)$ and aluminum $\left(932^{\circ} \mathrm{K}\right)$ to make this feasible.

\subsection{Engine Operation}

Start-up, steady cycling, and shut-down comprise the three phases of laser engine operation. In outer space, the temperature of the engine will become very low when it is not operating, and to conserve stored energy, all of the components will be idle, too. When it is tine to receive laser energy and begin operation, the bearing lubricants must be effective at the low station-keeping temperature, even before they are warmed up by friction and heat transfer. Window and engine jacket cooling can be effective immediately with gaseous coolants, as designed. Piston motion will be induced by the first pulse of absorbed laser radiation, but it may not be sufficient to produce a complete cycle, since nomally stored energy exists in the rotation of the flywheel to overcome 
all of the piston losses and still return the piston in time for the next laser pulse. Thus, it may be necessary to use the generator as a motor, initially, to start the engine. The energy required for start-up will be just the power needed to overcome motoring losses (about 0.2 kwatts) for the first few seconds of operation unt $i$ synchronism is established.

The simplest way to establish synchronisin is to vary the frequency of the starting motor so that it matches the timing of the incoming laser signal. A photo cell with a timing logic circuit to regulate the starting motor frequency may be required.

The engine cycle themodynamic variables have already been summarized in Table 17 . These values include the effects of heat transfer losses. Using the estimates of Section 2.2.2., the anticipated themal efficiency of this engine is 54 percent under optimized operation. With a generator efficiency of 95 percent, speed changer efficiency of 85 percent, and an optical component efficiency of 98 percent, the overall system efficiency is projected to be 43 percent. with improved piston seal lifetines, higher rpm operation would allow the sperd changer to be eliminated, improving the system efficiency to 51 percent.

At present, the cycle has only been analyzed so as to attain relatively high efficiency for operation with a $\mathrm{CO}_{2}$ laser and at low speeds of revolution. For higher temperature operation, for example with a co laser, higher compression ratios are called for according to the results shown in Figure 17. Further, if a inear generator were to be used, then a faster rom engine would be desirable in order to take full advantage of linear generator design efficiencies.

\subsection{Experimental Program}

Previous laser heat engine experiments lave explored the scientific feasibility of making a laser heat engine rum, but have not provided a systematic data base for optimizing the design of such engines in terils of their potential for high efficiency operation. The object of the proposed experimental program is to demonstrate the potential for overall operating efficiencies in excess of 50 percent. To do this, the major components of the laser heat engine system must be present in the experiment. That is, the haser, window, heat encine, working fluid/absorber, and generator must be connected and operating in unison.

While previous experiments conducted at NASA-Ames Research Center and elsewhere $[6,9]$ have had many of the components of a total laser heat engine systern operating together, those experiments did not provide convincing evidence that a laser heat engine device could be operated at high efficiency. 
It may still be impossibie to demonstrate the theoretically predicted peak efficiencies for a relatively small machine because of the way energy losses scale with size. For example, heat transfer to the walls in a small demonstration machine may dominate the entire thermal behavior of the device. Thus, the emphas is of the experiment should be a careful study of energy losses to document the scaling laws for these phenomena.

At the opposite extreme, a full-scale prototype device must be able to demonstrate the desired peak efficiencies. In that case, emphas is would be on a direct measurement of efficiency rather than on detailed diagnostics of the therinal cycle losses. Of course, a whole range of intermediate cases exist in which some of the device attributes may be inferred directly from experimental demonstration, and other attributes demonstrated indirectly by referring to scaling laws established by the experimental measurements.

The one-kilowatt 0tto/Diesel cycle laser heat engine chosen for experimental demonstration is the largest heat engine that can be driven by available $\mathrm{CO}_{2}$ lasers.* $A$ snlaller size engine would be completely overcome by the scaling problems alluded to above. Heat losses for the 1-kW piston engine are predicted to be on the order of 30 percent of the input energy. Hence, these losses should certainly be a measurable effect, and yet the overall cycle efficiency predicted for this engine is still projected to be close to the original objective, approximately 43 percent.

We recommend that experimental emphasis be on a careful diagnos is of the energy losses and the correlation of these measurements to obtain an accurate understanding of the device efficiency. The achievenent of high efficiency is also recomilended as a major objective for this demonstration program. gram are:

Other specific experimental goals for the demonstration pro-

- Achievement of peak temperatures in a laser energy absorption cell using optimal mixtures of helium, argon, and $\mathrm{D}_{2} \mathrm{O}$.

- Measurement of piston engine heat transfer losses and verification of a reliable hea transfer computational model.

\footnotetext{
*If the restriction of using an existing laser facility were relaxed, the laser heat engine recommended for demonstration might be very different. Further, a 10 to $20 \mathrm{~kW}$ laser tailored to the proposed use can probably be built for $\$ 200,000$ to $\$ 300,000$.
} 
- Measurement of window reradiation losses and verification of a reliable radiation computational model.

- Measurement of frictione: and pumping power loses and correlation of data tu appropriate scaling models.

- Identification of a viable piston sealing scheme which is extendable to space engine lifetimes.

- Identification of ?ubricants for the crankcase bearings and study of lubricant interactions with working fluid/absorbar operation.

Clearly, these goals are anbitious, and a multi-year program will be needed to achieve them. It: is important to establish priorities among these goals, so that at each stage definite milestones can be observed which will provide decision-making data on whether and how to proceed.

Further consideration of previous experiments reveals that the uncertainty surrounding the engineering feasibility of high efficiency laser heat engines is due to a lack of fundamental empirical knowledge. For example, it is not well known how hot a diamond window may be run over long periods of time. The AVCO experiment [39] suggests that steady thermal conditions were achieved at much higher $\mathrm{CW}$ intensities than the laser heat engine experiment would require, but no periodic stresses were applied and the total time of heating was on the order of ten seconds or less. Similar uncertainties exist with respect to the peak temperatures achievable in the $\mathrm{D}_{2} \mathrm{O}$ absorber and the extent of serious reradiation losses. Heat transfer calculations for piston engines are also an imperfect art, so that there are unresolved theoretical questions regarding these losses, too. In short, detailed diagnostics are essential to success in this experinent, and careful attention to possible adjustments in the design and operation of the engine throughout the experiment will help to circumivent barriers to the achieventent of high efficiency.

We propose the following tasks to address the goals of the demonstration program:

Task 1: Detailed Engineering Analysis and Preliminary Design

This task covers the development and adaptation of analytical techniques for the entire proposed program, including the design and evaluation of engineering tests, a detailed engineering analysis of the engine concepts developed in this report and a 
preliminary design of the experimental englne. Several important design choices must be made including the use of a linear or rotary generator, the type of piston seal ing and the window cooling technique. A second part of this task is to convert the conceptual design developed for the laser heat engine in this report into a more definitive concept.

\section{Task II: Engineering Tests}

A number of engineering tests should be performed during the early stage of this program to help in the final design and to verify several of the conceptual design features before the complete engine system is butlt.

1. Measure $\mathrm{CO}_{2}$ laser radiation absorption and consequent heating in absorber/working fluid mixtures. Several absorbers such as $\mathrm{D}_{2} \mathrm{O}$ and $\mathrm{SF}_{6}$ as well as working fluids (helium and argon) should be investigated in a cell which can be heated by a moderate power $\mathrm{CO}_{2}$ laser.

2. Verify the dependence of bleaching on laser pulse length: Since bleaching is a lower pulse length 1 imit and pulsed $\mathrm{CO}_{2}$ lasers naturally operate near this limit region, an experimental definition of this limit in a realistic geometry is important.

3. Determine the maximum temperature achievable with a realistic laser pulse, approximately $250 \mathrm{~J}$ in about $6 \mathrm{msec}$. Since the actual $\mathrm{CW}$ laser experiments will involve utilization of a complex and relatively expensive experiment, the use of a single pulse laboratory laser to precisely simulate the heating process is a very important feature of the program. Consequently, the laser absorption process can be fully understood when the actual laser/engine test program starts.

4. Measure transient thermal and pressure stresses in window structure due to laser heating of the gas sample. Coupled to Subtasks 1 and 3 above, the window can be instrumented to allow measurement of the transient thermal stress and pressure pulses.

5. Measure steady heat transfer capabilities of the window with active cooling. By proper simulation a low power laser can be used to simulate the heat transfer on the window structure to test the relatively complex window cooling scheme. 


\section{Task III: Final Engine Design}

Based on the results of Tasks I and II, both completed and ongoing, the final detailed design of the laser heat engine can be performed. This design phase must include all aspects of coupling the engine to the laser facility chosen as well as to the preiliminary test equipment and diagnostics.

Task IV: Construction and Engine Assembly

All non-existing components of the proposed experiments, including the engine components, instruments, transfer optics, and other ancillary hardware must be fabricated as designed in Task III. This task will culminate in the assembly of all the engine components and check-out prior to motoring tests.

Task V: Diagnostics and Controls Development

In view of the emphasis of the proposed program on understanding the loss mechanisms and sealing, a careful diagnostic effort will be required. Consequently, we propose a separate task to develop, test, and fabricate the diagnostics concurrently with the engine experimental hardware design and fabrication. This effort should include diagnostics and controls for:

1. Pressure, mass flow, and temperature of the working fluid and coolants.

2. Cylinder wall tenperatures.

3. Engine speed.

4. Power input (motoring) and output (generated or torque).

5. Gas sampiting.

6. Chopper for $\mathrm{CW}$ laser and chopper speed monitor.

7. Start-up and shut-down controls.

\section{Task VI: Motoring Tests}

Prior to demonstrating the performance of the laser heat engine with the laser facility, a number of tests can be performed which will greatly enhance the analysis and interpretation of 
the data as well as simplify the test program involving hot laser-on tests. In these tests the laser heat engine is driven by an electric motor. Such tests should:

1. Measure frictional losses (valves and manifolds held open).

2. Measure pumping power and heat transfer losses (valves and manffolds operating normally -- preheated gas stream).

3. Measure static and dynamic piston ring sealing losses.

4. Test gas line tuning.

5. Verify mechanical integrity with full pressure and temperature loading.

6. Conduct single pulse laser tests to attain and verify full pressure and temperature load history.

\section{Task VII: Laser Engine Demonstration Tests}

The laser engine demonstration tests should be conducted, in conjunction with a laser facility discussed in the next section, in a three-step program.

1. Initial development tests to gain understanding of the operation of the laser engine and to check and verify the operation of all the diagnostic equipment.

2. Measurement of operating efficiency of the laser heat engine operated at or near the optimum design point.

3. Determination of the engine performance over a maximum range of conditions including variation of:
a. Load
b. Input gas pressure
c. Working fluid/absorber composition
d. Engine cycle:/laser phase
e. Laser power and pulse duration. 
Task VIII: Reports

A program of this nature should be reported on a quarterly basis with a detailed technical report due at the end of the program. Special topical reports may be desirable on separate events or phases of the program.

The objectives of the demonstration program should be to verify the design efficiencies predicted for the piston engine. The first tests may be open-cycle and short duration, although longer duration tests (e.g., on the order of minutes) may be desirable for a convincing demonstration of overa 11 engine efficiency. While it may not be possible to completely achieve the desired 50 percent peak efficiency, the program should be formulated in terms of reaching a definite level of performance. We believe that a reasonable goal would be the achievement of 40 percent overall efficiency (laser energy in to el ectricity out) or at least wi th simulation of the ancillary losses from standard commercial equipment. Some redesign may be necessary after the first experiments in order to reach peak efficjency. A preliminary project schedule is shown in Figure 38.

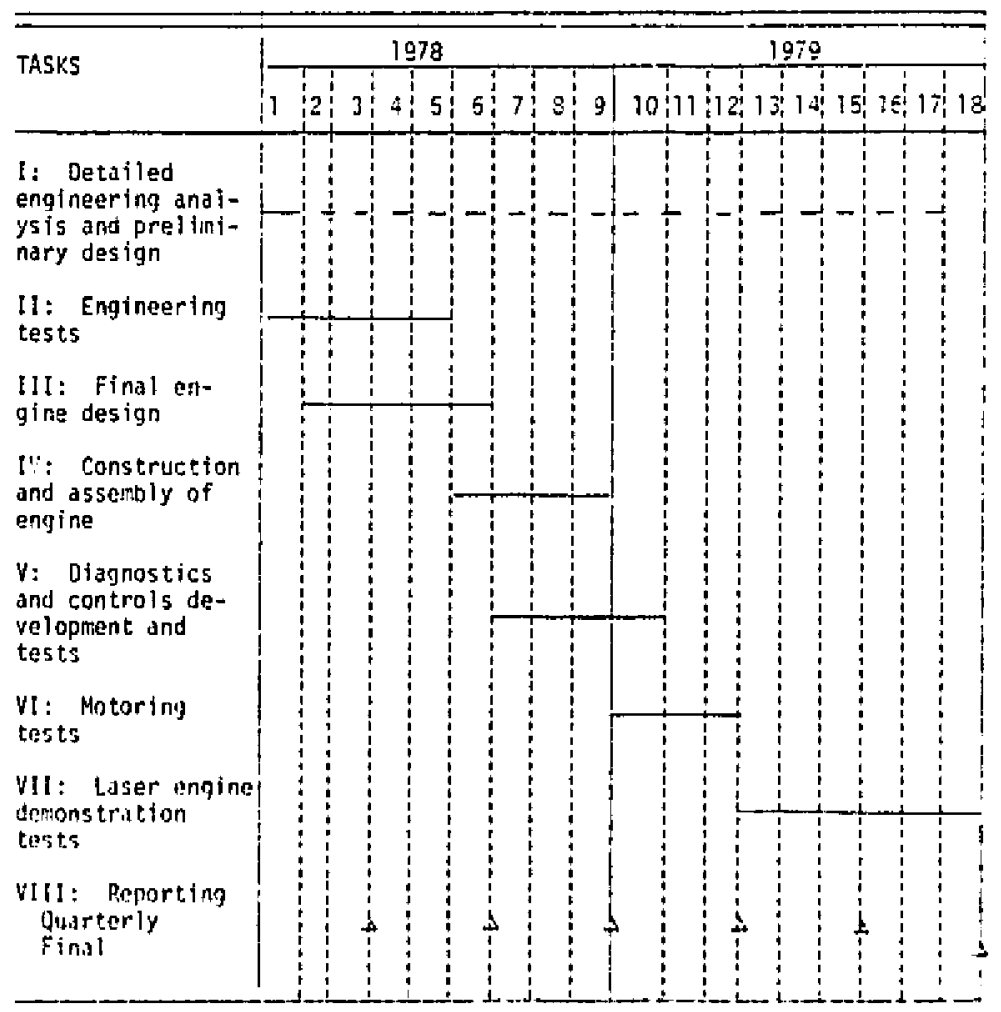

Figure 38. Tentative Schedule for Laser Heat Engine Experimental Demonstration 


\subsection{Site and Facillty Requirements}

Site selection required careful consideration of the availability of hardware and facilities, the cost of utilizing existing hardware and its modification, the new hardware to be produced, the limitations on the demonstration created by the facility (be it existing or to be constructued), and the integration of the program with NASA technical-and mission-oriented staff. Consequently, this process was an evaluation during which many sources were consul ted.

The existence of a suitable laser for the demonstration experiments is by far the dominant site-specific requirement. An average laser power absorbed of two to four kilowatts is needed for the onekilowatt output engine. This power must be delivered in less than five percent of the engine cycle period, once every cycle. At $500 \mathrm{rpm}$, 5 percent of the cycle period is 6 milliseconds. Therefore, the actuai laser power required must be at least $40 \mathrm{kilowatts} \mathrm{CW}$ or, al ternately, 240 joules per pulse, with a maximum pulse length of $6 \mathrm{mill}$ iseconds at 8.3 pulses per second. Such a repetitively pulsed laser does not exist, but could be built. However, a large $\mathrm{CW} \mathrm{CO} 2$ laser in the $50-\mathrm{kW}$ size range exists at NASA-Ames. The Ames laser is a blow-down facility with a 15-second run time. At NASA-Lewis a $15-\mathrm{kW} \mathrm{CO}_{2}$ laser facility exists. The Lewis facility is a continuously operating, closed cycle, electric discharge laser with run times exceeding several hours. Although it has the potential of higher power, it has so far produced only $15 \mathrm{~kW}$ of power.

The second class of items whose availability affects the choice of demonstration site are major heat engine components and test facifities. These include pumps, turbines, heat exchangers, dump tanks, and effluent treatment facifities (in the case of an open-cycle demonstration). Not so critical, but still important, are test facilities such as dynamic balancing equipment (for rotating machinery), precision machining facilities, engine operation test equipment (e.g., dynamometers), and so forth. Most of these items are available at any major national laboratory, so that their availability and location is not important to choosing the site of the experimental program.

Another important consideration in choosing the site is the ease with which the relevant technology can be integrated with NASA's mission. Proximity to NASA personnel involved with the demonstration project and the channels of communication between the NASA staff and the contractor personnel are of primary importance. Both on- and offsite demonstration project al ternatives were considered from the point of view of the effects that these alternatives have on technology transfer. Other considerations being equal, the greatest ease of communication is achieved by having the prime contractor and the demonstration project operate at a NASA facility. 
On the basis of laser availability at the CW power level required, it appears that the demonstration experiments can best be carried out at the NASA-Ames research facility. NASA-Ames has the necessary auxiliary facilities as well as staff, and is in a good position to cooperate with contractor personnel. The only drawback of the NASA-Ames facility is the limited run time. However, thermal balance can surely be achieved either directly or by preheat of critical areas. NASA-Ames personnel are already experienced in the technology of laser heat engines and consequently are in a good position to support such a program.

A program could also be performed at NASA-Lewis, however the performance may need to be modified. It appears that using a longer laser pl.ise, possibly as long as $15 \mathrm{msec}$ and lasting 15 percent of the cycle time, would allow the experiment to be performed at a 15-kW power level. A lower power experiment, i.e., less than 1-kilowatt output power) could also be planned. However, in view of the lower power, the Lewis facility would not present as much experimental flexibility. of course, the advantage of long run times clearly speaks for the Lewis 1aser.

Al ternatively, the experiments could be performed at ans $s *$ te, national laboratory or contractor facility, if the specification of an existing laser is relaxed. Several commercial high power closed cycle lasers exist in the $1-$ to $15-\mathrm{kW}$ class costing approximately $\$ 40 /$ watt as complete systems. The flow loop and optical cavity of one of these lasers could be modified for pulsed or quasi-steady operation with several hundred joule/pulse capability. We estimate that such modifications (including the basic flow loop, power supplies and optical cavity) can be acquired for about $\$ 250,000$.

The balance of the program, other than the laser engine demonstration experiment, does not require any special facilities other than the assortment of laboratory equipment and, in particular, lasers to perform the engineering and development testing.

\subsection{Longer Range Laser Heat Engine Program}

During the course of this study it became clear that the choice of heat engine type might be quite different if the groundrules were different, specifically, if the choice were not restricted by current laser availability for a laboratory demonstration and if the desired power level were considerably larger. Further, it was also clear that some of the space mission requirements may have a strong affect on the eventual choice of a preferred technology. In view of the serious implications of this potential, we also considered a longer range laser heat engine development program which is designed to answer 
questions not currently addressed, and not within the scope of the proposed near term denonstration. These questions fall into two general categories: technological questions about the potentiai of the Brayton/Energy Exchanger/Co Cycle, and questions about systems implications. The following paragraphs suggest programs to illuminate these areas.

\subsubsection{Brayton/Energy Exchanger/CO Cycle}

In view of the superior efficiency potential of this cycle, a series of steps designed to further evaluate the potential of this technology appears warranted. One aspect of this question, the development of an efficient high temperature energy exchanger as a cycle element for various heat engine cycles, will most probably proceed during FY 78 and 79 under Department of Energy (DOE) sponsorship. DOE is currently negotiating such a program with MSNW. To keep pace with this technology we recommend a program to:

1) Define the optimal co laser characteristics for a Brayton/ Energy Exchanger/Co Cycle system and perform a preliminary design analysis of such a laser.

2) Perform absorption experiments in an absorption ce11 with a CO laser tunable to various individual lines.

3) Design a Brayton/Energy Exchanger/Co laser heat engine experiment.

\subsubsection{Systems Implication of Laser Heat Engines}

Since the future direction of any laser heat engine technology program depends strongly on the space applications most likely to become realjties, a program to assess the impact of the space systems applications on the technology is highly desirable. Such a program or study is important so that the demonstration proposed in the previous paragraphs can be followed by properly directed programs of technology development which will yield the type of system which the applications demand. Such a study should consider the following:

1) Mission analysis--What are the most useful power levels for future space missions and how is that level likely to change with time? What are the advantages of laser heat engines as power systems and under what circums tances are they likely to be used in preference to other space power sources? How long will a laser heat engine have to operate without attention? What lubrication and sealing systems are consistent with the above? 
2) Technology questions-Are all the components required for various systems readily avaflable? When are various improvements in technology likely to be available to laser heat engines? Are there other technologies which could make significant improvements in the current laser reat engine concepts?

3) Program recommendations--Based on the above studies and the demonstration program, a long-range program, both for technology development as well as for prototype development, should be recommended to realistically assess when this technology could actually be utilized in space. 


\section{SUMMARY OF RECOMMENDATIONS AND CONCLUSIONS}

\subsection{Near-Term Demonstration Program}

At very small power levels, piston engines have both nearterm technology and efficiency advantages compared to turbine engine systems. Of the piston engines, the otto/Diesel cycle, two-stroke engine appears superior because of extensive experience in the construction and operation of such engines, and because of an estimated efficiency advantage compared to stirling engines once the multiplicative effects of monatomic gas operation are accounted for. Thus, the principal recommendation of this study is to use the Otto/Diesel cycle piston engine for a near-term experimental demonstration program. Detailed recommendations have been made throughout the text. The highlights of these are:

1) To carry out the demonstration program at the NASA-Ames Research Center because of the availability of the required high power $\mathrm{CO}_{2}$ laser facility.

2) To pursue the demonstration program with a central contractor who has all the necessary experience and equipment to do the design, construction, and preliminary testing at their facility.

3) The basic goals of the demonstration program should be to establish open-cycle operation of the 1-kW engine at high thermal efficiency. The program should also develop an explicit understanding of engine operation necessary for improving engine performance through redesign, better materials, etc. to the predicted overall efficiency of over 40 percent.

4) The demonstration program must also document the major scaling laws for losses in efficiency.

\subsection{Development Program for Large-Scale. Laser Heat Engines}

A second major result of this study has been that the turbine/ energy exchanger, laser heat engine appears to be the most promising device for larger, efficient laser heat engines. Development of the energy exchanger component efficiency will be desirable in order to achieve the full advantage of this type of engine. However, if 
sufficient laser power existed today, it would be encirely feasible to build and operate a high efficiency, turbine/energy exchanger laser heat engine with present technology, above the 100-kilowatt power teve1. This type of engilie requires a specially tuned co laser for its most efficient operation. Co lasers at this power level exist now, but their run times are too short for reasonable experimentation. The results of this study indicate that the redesign of $\mathrm{CO}$ lasers to match the energy absorption requirements of the turbine/energy exchanger type of laser heat engine is an attractive high potential payoff approach. 


\section{REFERENCES}

1. Laser Energy Conversion Symposium, ed. K.W. Billman, NASA TMX-62, 269, 18-19 January 1973.

2. Second NASA Conference on Laser Energy Conversion, ed. K.W. Eillman, NASA SP-395, 27-28 January 1975.

3. R.C. Weatherston and A. Hertzberg, "The Energy Exchanger, A New Concept for High Efficiency Gas Turbine Cycle," J. Eng. Power (1966).

4. A. Hertzberg, "The Energy Exchanger for Use in Laser Heat Engines," Ref. 2, op. cit.

5. M. Garbuny and M.J. Pechersky, "Laser Engines Operating by Resonance Absorption," Appl. Optics 15, 1141-1157 (1976).

6. R.L. Byer, "Initial Experiments with a Laser Drive Stirling Engine," Ref. 2, op. cit.

7. W. R. Martini, "Space Electric Power Design Study," Contract A-29674B, NASA-Ames Research Center.

8. C.F. Hansen and G. Lee, "Laser Power Stations in Orbit," Astronautics and Aeronautics, July 1972, pp. 42-55.

9. G. Lee, private communication, October 1977.

10. A.J. Streb, "Radioisotope Power Systems for Manned Space Stations," pp. 3-29 in Space Power Systems Engineering, ed. G.C. Szego and J.E. Taylor (Acadenic Press: New York, 1966).

11. A.D. Tonelli and T.C. Secord, "Auxiliary Power Generating System for a Large Space Laboratory," pp. 299-322, Ref. 10, op. cit.

12. P.G. Hill and C.R. Peterson, Mechanics and Themodynamics of Propulsion (Addison-Wesley Pub7. Co.: Reading, Mass., 1965), Chapter 12 .

13. C. SeippeI, "Pressure Exchanger," US Patent No. 2,399,394 (1946).

14. W.E. Smith and R.C. Heatherston, "Studies of a Prototype Wave Superheater Facility for Hypersonic Research," Cornell Ae*'onautica) Laboratory Report HF-1056-A-1-AF0SR-TR-58-158, AD207244. Decenber 1958. 
15. K. Kunberger, "Progress in Comprex Supercharger Development," Diesel and Gas Turbine Progress (Worldwide Editions, 1973).

16. M. Berchtold and T.W. Lutz, "A New Small Power Output Gas Turbine Concept," ASME Paper No. 74-GT-111, Gas Turbine Conference and Products Show, Zurich, Switzerland, 1974.

17. R. Taussig, High Thermal Efficiency, Radiation Based, Advanced Fuston Reactor, Mathematical Sciences Northwest, Inc. Report No. MSNW-77-1067-1, Apri1 1977.

18. R.C. Weatherston, W.E. Smith, A.L. Russo, and P.V. Marrone, Gasdynamics of a Wave Superheater Facility for Hypersonic Research and Development, CAL Report No. AD-1-118-A-1, February 1959 .

19. Sma11 Gas Turbines for Helicopters and Surface Transport, Lecture Series No. 46, ed. J. Fabri, AGARD-LS-46-71 (Tech. Editing and Reproduction, Ltf.: London, 1971).

20. M.G. Coombs and L.W. Norman, "Application of the Brayton Cycle to Nuclear Electric Space Power Systems," pp. 419-482, Ref. 10, op. cit.

21. D.G. Elliot, D.J. Cerini, and E. Weinberg, "Liquid-Metal MHD Power Conversion," pp. 1275-1298, Ref. 10, op. cit.

22. J.R. Kessler, "Hyperbolic Field Reciprocating Space Power Unit," pp. 1197-1212, Ref. 10, op. cit.; H.M. Cameron and W.E. Morgan, "Development of Hydrogen-0xygen Fueled 3-kW Internal Combus tion Engine," pp. 1213-1234, Ref. 10, op. cit.

23. L.C. Lichty, Combustion Engine Processes (McGraw-Hill Book Co.: New York, 1967), Section 15-46 to 15-53, pp. 543-547.

24. L.C. Lichty, pp. 528-529, Ref. 23, op. cit.

25. W.H. Perciva1, Historical Review of Stirling Engine Development in the United States from 1960 to 1970 , NASA CR-121097, prepared for ERDA, July 1974, EPA Contract 4-E8-00595.

26. D.A. 0liver, M. Mitchner, "Nonuni form Electrical Conduction in MHD Channels," AIAA J. 5 , 8 (1967); R. Decher, "MHD Generation Characteristics with Insulator Wall Losses," AIAA J. 8,1 (1970); M.A. Hoffman, "Nonequilibrium MHD Generator Losses Due to Electrode Wall and Irsulato" Wall Leakages," AIAA J. 6, 9 (1968). 
27. J.L. Kerrebrock, R. Dethlefson, "Experimental Investigation of Fluctuations in a Nonequilibrium MHD Plasma," AIAA 3. 6,11 (1968).

28. R. Decher, J.L. Kerrebrock, M.A. Hoffman, "Behavior of a Large Nonequflibrium MHD GEnerator," AIAA J. 9, 357 (1971).

29. J.F. Louis, "Effective Ohm's Law in a Partially Ionized Plasma with Density Fluctuations," Phys. Fluids 10, 9 (1967).

30. A. Solbes, "Quasi-Linear Plane Wave Study of Electrothermal Instabilities," Electricity from MHD, Proceedings of the Warsaw Conference, July 1968.

31. M.A. Lutz, "Radiation and its Effects on the Nonequilibrium Properties of a Seeded Plasma," AIAA J. 5,8 (1967).

32. A.L. Hoffman, App1. Phys. Lett. 23, 693 (1973); H.L. Rutkowski, D.W. Scudder, A.Z. Pietrzyk, and G.C. Vlases, Appl. Phys. Lett. 26, 421 (1975).

33. B.J. Klein and J.J. Degran, "Optical Antenna Gain 1: Transmitting Antennas," Appl. Optics 13, 9, 2134 (1974); B.J. Klein and J.J. Degran, "Optical Antenna Gain 2: Receiving Antennas," Appl. Optics 13,2, 2397 (1974); these references are only one example of many recent papers on this topic.

34. D.L. Nored, "Applications of High Power Lasers to Space Power and Propulsion," pp. 95-108, Ref. 2, op. cit.

35. T. Vera and T. Asakura, "Apodization for Maximum Encircled Energy with Specified Overall Transmittance, J. Optics (Paris) 8 , 1, 15 (1977).

36. G. Lansraux and G. Boivin, Canadian J. Phys. 39, 158 (1961).

37. W.B. Bridges, et al., "Coherent Optical Adaptive Techniques," App 1. Optics 13, 2, 291 (1974).

38. T.J. McCusker, "Solar Concentrator Design and Construction," pp. 687-700, Ref. 10, op. cit.

39. J.L. Zar, Investigations into the Feasibility of High Power Laser Window Ma terials, Selli-Annual Technical Report No. 2, AVCOEverett Report No. AFCRL-TR-75-0605, Oc tober 1975; D.H. DouglasHamilton. E.D. Hoag, and J.R.M. Seitz, "Diamond as a High Power Laser Window," Optical Soc. Am. 64, 1, 36-38 (1974). 
40. F. Horrigan, C. Klein, R. Rudko, and D. Wilson, "Windows for High Power Lasers," Laser Technology Section (Laser Optics: Danbury, Conn., January 1969), pp. 68-76.

41. American Institute of Physics Handbook, 3rd Edition, ed. D.E. Gray (McGraw-Hi17, New York, 1972), Chapter 6; Handbook of Military Infrared Technology, ed. W.L. Wolfe (Office of Naval Research: Washington, D.C., T965), Chapter 8.

42. G.E. Caledonia, "Conversion of Laser Energy to Gas Kinetic Energy," J. Energy 1, 121-124 (1977).

43. S.J. Holmes, private communication, 1977.

44. J.W. Daiber and H.W. Thompson, "Performance of a Large CW Preexcited CO Supersonic Laser," IEEE J. Quant. Elect. QE-13, 10 (1977).

45. "A Study Optimization and Design of a Laser Heat Engine," MSNW Proposal 1641, submitted to NASA-Ames Research Center May 6, 1977.

46. J.F. Zumdieck and S.A. Zlatarich, "Decomposition of Nitric Oxide in a Hot Nitrogen Stream to Synthesize Air for Hypersonic Wind Tunnel Combustion Testing," NASA Technical Note TN-D7849, NASALewis Research Center, December 1974.

47. D.R. Stull and H. Prophet, JANAF Thermochemical Tables, 2nd Edition, United States Department of Commerce, National Bureau of Standards (1970).

48. A.H.M. Ross, R.S. Eng, and H. Kildal, "Hetrodyne Measurements of CO Isotope Laser Frequencies: Mass Dependence of Dunham Coefficients," Optics Communications 12, 433-438 (1974).

49. A. Mitchell and M. Zemansky, Resonance Radiation and Excited Atoms (Cambridge University Press: Cambridge, 1961).

50. G. Hertzberg, Spectra of Diatomic Molecules (Van Nostrand: New York, 1950).

51. P. Varanasi, "Measurement of Linewidths of $\mathrm{CO}$ in Atmospheres of Planetary Interest at Low Temperature," J. Quant. Spect. Rad. Trans. 15, 191 (1975).

52. N. Djeu and S.K. Searles, "Method for Measuring Relative Transition Probabilities of Cascading Molecular Bands: Application to CO Fundamental Bands," J. Chem. Phys. 57, 4681-4687 (1972). 
53. S.S. Penner, Quantitative Molecular Spectroscopy and Gas Emissivities (Addison Wesley: Boston, 1959).

54. R.C. Millikan and D.R. White, J. Chem. Phys. 39, 3209 (1963).

55. D.J. Miller and R.C. Miliikan, J. Chem. Phys. 53, 3384 (1970).

56. J. Willfamson, Ph.D. Thesis, Ohio State University, unpublished (1969).

57. F. Keilman et a1., Appl. Phys. Lett. 26, 1 (1975).

58. A. Ferriso et al., JQSRT $\underline{6}, 241$ (1966).

59. L.A. Gribov and V.N. Smirnov, Sov. Phys. Uspekhi 4, 942 (1962).

J. B. Goldwater, S. Piller, J. Rauch, and A. Cella, Demonstration of a Free-Piston Stirling Engine Drive Linear Al ternator: Phase I March 1977.

61. D.B. MacKay, Design of Space Power Plants (Prentice-Hall, Inc.: Englewood Cliffs, N.J., 1953).

62. I.J.Leoff]er, S. Lieblein, "Materials Problems Associated with the Design of Radiators for Space Power Plants," pp. 627-653, Ref. 10, op. cit.

64. A.D. Turrin and A. Blau, "Solar Dynamic Power Systems from 3 to $100 \mathrm{~kW}, "$ pp. 703-731, Ref. 10, op. cit.

65. Kodak vendor literature.

66. M.R. Townsend, "Direct Delivery of Automated Spacecraft Using the Shuttle: Thoughts for the Designer," Astronautics and Aeronautics 15, 32 (1977); C.J. Daros, R.F. Freitag, and R.L. Kline, "Toward Large Space Systems," Astronautics and Aeronautics 15, 22 (1977).

67. W.R. Corliss, "Survey of Space Power Requirements--1962 to 1976," pp. 3-13 in Power Systems for Space Flight, ed. Z.G. Szego (Academic Press: New York, 1962).

68. F.J. Cepollina and E.I. Pritchard, "STS Multimission Modular Spacecraft--A New Horizon in Social and Industrial Benefits, "Astronautics and Aeronautics 15. 36 (1977).

69. R.L. Merritt and B.W. Davis, "Shuttle Payloads: 0pportunities to Expand Ground-Based Research," Astronautics and Aeronautics 15, 38 (1977). 
70. E. Schne11, "Analys is of Sma11 Gas Turbine Components," pp. 4-1 to 4-23, Ref. 19, op. cit.

71. A.L. Jaumotte, "Application to Power Generation," pp. 6-1 to 6-25, Ref. 19, op. cit.

72. D.L. Southam, "Power Systems Comparison for Manned Space Station Applications," pp. 335-360, Ref. 10, op. cit.

73. R.R. Paxton and W.R. Shobert, "Testing Carbon for Seals and Bearings," J. Amer. Soc. Lubrication Engr. 1, 27 (1961).

74. H.D. Lamping, M.W. Galliers, and S.M. Wolosin, "Rotary Combustion Engine Trochoid Coatings and Seals," SAE Paper No. 741043, Automobile Engineering Meeting, Toronto, 21-25 October 1974. 